Angelo Chiuchiù - Giuseppe Asciak Pace - Marion Asciak Pace

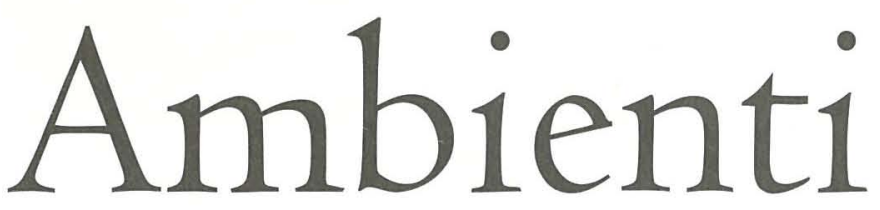

\title{
Percorsi letterari del '900 italiano
}

\section{Prefazione di Renzo Pavese}
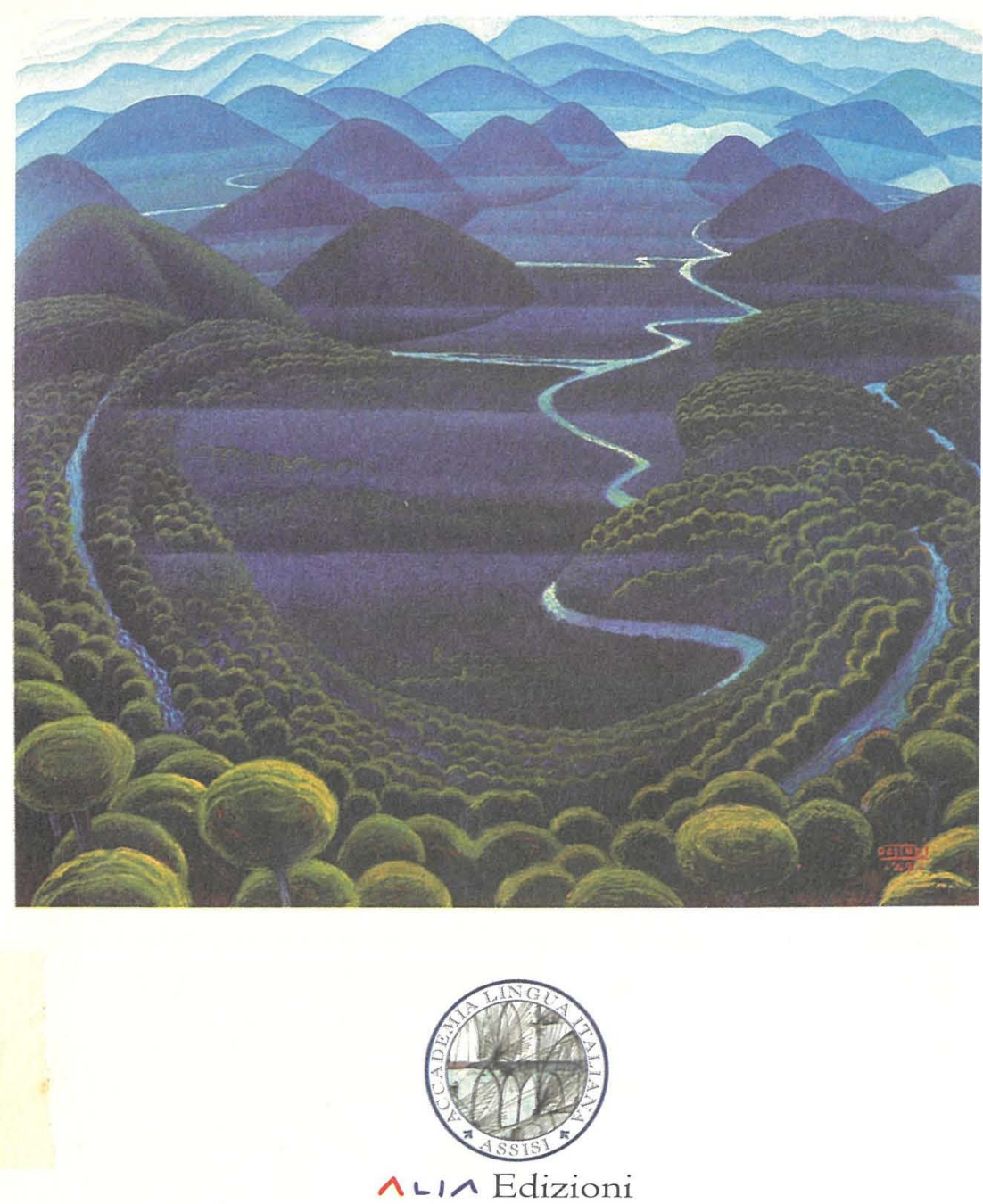
Angelo Chiuchiù - Giuseppe Asciak Pace - Marion Asciak Pace

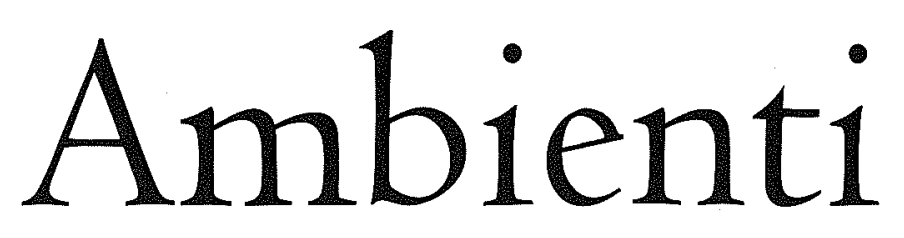

\section{Percorsi letterari del '900 italiano}

Prefazione di Renzo Pavese

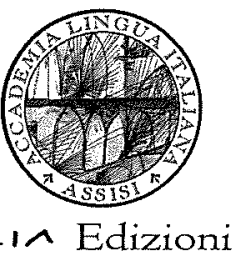


Copertina di Lorenzo Chiuchiù

Quadro in copertina di G. Dottori, Umbria Vergine, 1949

(C) 2001 Alia Edizioni Assisi

Tutti i diritti riservati sia del testo che del metodo

Gli autori e l'editore sono a disposizione per quanto riguarda il copyright degli aventi diritto non rintracciati. 


\section{PREFAZIONE}

In questo originale manuale per l'insegnamento dell'italiano a livello avanzato, dal titolo significativo ed esplicativo Ambienti. Percorsi letterari del' 900 italiano, gli autori hanno cercato un punto di intesa, o di passaggio, tra il linguaggio letterario e quello per così dire pratico, al fine di cogliere e trasmettere sensazioni, atmosfere, colori di paesaggio e d'anima italiani utili per lo straniero, e non solo. Mediante riflessioni su testi tratti da opere famose pubblicate nella prima parte del Novecento e negli anni Sessanta e Settanta, per essere precisi, si vuole spingere l'allievo, o il libero discente, ad allenarsi a individuare, a confrontare e ad apprendere espressioni consuete e non sempre consuete nel modo di parlare e di scrivere contemporaneo o attuale, cioè espressioni sempre illustranti tuttavia cultura, grammatica e sintassi italiana, che vengono del resto spiegate a piè di pagina in casi di difficoltà.

I testi, secondo l'ordine di successione che compare nel manuale, sono tratti da noti autori quali Natalia Ginzburg, Guido Piovene, Umberto Saba, Giuseppe Tomasi di Lampedusa, Giuseppe Marotta, Ignazio Silone, Giorgio Caproni, Luigi Pirandello, Giorgio Bassani, Carlo Emilio Gadda, Dino Campana.

Nel brano della Ginzburg si parla di Walter, un tipo alquanto "avulso dagli altri esseri umani", anche se bello e attraente al punto da suscitare "l'amore in molte donne", che del resto lui disprezzava. Chi parla di Walter è un amico, che ne traccia un profilo unendo il passato al presente: Walter è sposato, ha un figlio ed ha invitato l'amico - che è l'io narrante - a raggiungerlo "in una città balneare, dove egli si trovava con la moglie e il bambino". Si apprende altresì che le lettere di Walter, inviate all'amico, 
sono "puerili e sgrammaticate" e si insiste sulla sua indolenza, sui suoi silenzi, sulla sua mancanza di interessi e sulla sua indifferenza di carattere che lo porta a rendersi indisponibile verso gli altri.

Il brano di Piovene, invece, ci pone di fronte alle caratteristiche dell'Umbria: "aspetto tranquillo", "bellezze artistiche e naturali", "bel paesaggio", "ricordi francescani", una terra che lascia un'impressione "molto gradevole" negli stranieri. Se la "bellezza toscana", in arte, è "rigorosa e astratta", la bellezza umbra "è morbida, più stemperata e più sfumata". Le città umbre sono poste "in altura" o sulle "pendici"; ci sono "rocche", "oliveti", "suoni di campane e rondini". Assisi predomina su tutte le altre città, con le sue "numerose terrazze", con i suoi "panorami idillici", con la sua storia artistica e devota legata a San Francesco.

Con Saba abbiamo uno sguardo su Trieste e le sue tre vie principali, su case, magazzini, ospedale, convento, chiesa, camposanto, nonché ricordi e sentimenti d'amore.

Entriamo poi in un ambiente tipicamente siciliano con Tomasi di Lampedusa. C'è la campagna, la fattoria, una natura e un'atmosfera particolare per calura, siccità e polvere, dove i personaggi si muovono a disagio in una terra "arsa che alla fine di Agosto aspetta invano la pioggia", a $50^{\circ}$ di temperatura. Qui tuttavia spicca "la correttezza elegante di Tancredi" - uno dei protagonisti del romanzo - che sembra essere l'unico in grado di aiutare gli altri e di saper portare vita e vivacità in quel luogo, insieme al cane Bendicò, il quale prima abbaia alle funeree cornacchie e poi ha "impeti passionali" all'arrivo del giovane padrone.

Marotta ci dà l'ambiente e il paesaggio napoletano con scorci di vita in alcuni quartieri famosi di Napoli e riferimenti ad alcuni cibi tipici come le 
cozze, le alici e il brodo di polipo. Predomina un colore locale sia negli aspetti esteriori delle case e dei negozi, sia nei movimenti della gente nei vicoli, negli accenni al mare e alle barche.

Nel brano di Silone, poi, oltre alle fatiche dei contadini abruzzesi - i cosiddetti "cafoni" secondo il linguaggio dialettale, un termine che, come si $\mathrm{sa}$, è passato nella lingua italiana con il significato di persona rozza, villana o maleducata - viene sottolineata anche la precarietà di certe zone italiane che si trovano sempre in pericolo di frane, allorché vengono le piogge e il terreno coltivato viene portato via da "un'enorme fiumana" che rende nulli gli sforzi dei lavoratori per bonificare il terreno e il loro attaccamento alla terra. Oltre ai terreni, naturalmente sono anche le case che spesso scompaiono.

Nella poesia di Caproni si parla invece di un'escursione in montagna, dove "l'occhio" può "spaziare". Tuttavia c'è anche un invito a non "abusare" di marce impegnative, a "riprender fiato" "sul pianoro", a brindare alla riuscita senza però esserne troppo orgogliosi, perché c'è sempre da tener presente il "debito con la fortuna", c'è sempre ancora da passare "la cresta" dell'altura e non si sa che cosa ci sia dall'altra parte. In montagna ci sono "mormorii", "voci", "brusii" e prima di "avventurarci", si dice "godiamoci quindi insieme / l'insolita sicurezza". Il tutto può essere interpretato anche per metafora.

Con un brano tratto da Il fu Mattia Pascal siamo immersi in una tipica atmosfera pirandelliana. Il freddo dell'inverno incide sull'animo del protagonista, cioè l'animo di questi "prende qualità dal colore del tempo", anche se il protagonista cerca di opporsi. Si tratta di Adriano Meis, che altri non è che Mattia Pascal, il quale ha deciso di cominciare una vita nuova con 
un nuovo nome, soprattutto dopo che ha letto sul giornale la notizia del proprio suicidio (al suo paese infatti era stato identificato nel cadavere di un annegato suicida). Egli pensa di "diventare uomo" e di formarsi "un abito di vita quieto e modesto", per essere più "libero e senza obblighi di sorta". Anzi, egli vuole comporsi "una regolare esistenza" e così inizia ad immaginare città dove potrebbe abitare. Ora non desidera più vivere in un albergo con la precarietà del viaggiatore, ma in una casa anche modesta che sia tutta sua, attorniato da oggetti che rispecchino la sua anima e i suoi ricordi. Del resto si sta avvicinando il Natale, cioè il periodo in cui maggiormente si brama "il tepore d'un cantuccio caro", "l'intimità della casa". C'è tuttavia la preoccupazione in lui dell'iscrizione all'anagrafe, in quanto il suo nome è falso e lui teme guai con la polizia.

Con Bassani ci trasferiamo nei Lidi ferraresi o Valli di Comacchio ad osservare il volo dell'airone, un uccello dal corpo piccolo rispetto alle grandi ali. L'airone vola "arrancando", sembra "non farcela a tagliare di traverso il vento", poi sbanda perché colpito da una fucilata e cade quasi "rompendosi in tanti pezzi". Sembra morto, una cagna va a recuperarlo, ma ecco che l'uccello si rizza sulle gambe e comincia a muoversi in qua e in là con atteggiamenti da viveur, viene detto. Alla fine, però, è tutto uno scambiarsi di sguardi tra l'airone morente, vittima innocente, e il cacciatore colpevole di averlo colpito, tutto avvolto nella sua angoscia esistenziale. L'airone ha atteggiamenti umani : osserva, medita, soffre.

Con Gadda, quasi per un'associazione di idee, si passa alla tragica immagine di una donna morente. Siamo nel finale de La cognizione del dolore, romanzo non terminato dall'autore. C'è una donna anziana che è stata aggredita di notte ed è sul punto di morire. È più morta che viva, per tutta la notte hanno cercato di curarla, lei è stesa sul letto, è agonizzante, i 
suoi occhi sono aperti, guardano "il nulla". Ha sangue coagulato "sui capelli grigi", "due fili di sangue le colavano dalle narici", "la guancia destra [è] tumefatta", "le mani levate [sono] scheletrite". Il medico presta tutte le cure necessarie: dalle pezzuole strizzate si sente il gocciolare di "acqua" e "alcool" "in una bacinella". Intanto alla finestra, tra le persiane, appare l'alba, mentre il gallo canta e tutto intorno si diffonde il chiarore dell'aurora, soprattutto sui gelsi, in fila.

Il poeta Dino Campana conclude il repertorio di "ambienti" con immagini e sensazioni tratte dalle "solitudini mistiche" del convento sul Monte della Verna (in Toscana), dove San Francesco ha ricevuto le stigmate (o stimmate). Siamo nei luoghi sacri di San Francesco dove anche gli abitanti hanno dipinto in volto "un raccoglimento inconscio, una serenità conventuale". E poi c'è la luce del tramonto che si riflette sul paesaggio roccioso, sul convento, sui bassorilievi, sulla Vergine, all'esterno del monastero. All'interno, nella Cappella delle Stimmate, oltre ad esserci la presenza di San Francesco, s'aggirano profili ieratici e figure di santi. Fuori, nella penombra, regna il silenzio, il sogno, e lo sguardo vaga in lontananza, partendo da una mistica altezza.

Fin qui per quanto riguarda la letteratura e i suoi contenuti. Poi seguono i moduli e gli esercizi di lingua, gli inviti a meditare su strutture formali e lessicali di base, a cambiare e a modificare modi e tempi verbali, a riconoscere pronomi, a distinguere il concetto "vero" dal "falso", a lavorare sulle "giuste alternative". Si richiedono significati di parole, sinonimi e contrari, interpunzione giusta, articoli seguendo le regole, preposizioni e congiunzioni opportune, correzioni di errori, interpretazioni di modi di dire e metafore, scelte multiple. Insomma vengono messe a fuoco tutte le parti del discorso e i punti essenziali della grammatica e della sintassi al fine di 
preparare l'allievo, in modo appropriato e guidato, alla conoscenza approfondita della lingua italiana sia orale che scritta, attraverso una serie di esercizi non ripetitivi, a più moduli, attraverso una ricerca di significati vari, ora puntuali ora sfumati ora contrari, attraverso correzioni di sbagli e imprecisioni, attraverso il gioco e lo stimolo della curiosità.

L'opera è accompagnata da un cd che contiene la registrazione degli undici brani d'autore letti da Marco Brancato che dà ai testi un ritmo interpretativo intelligente ed efficace a seconda dei contenuti e delle situazioni narrate.

RENZO PAVESE 


\section{1 \\ CASA AL MARE}

Da molti anni non vedevo il mio amico Walter. Qualche volta lui mi scriveva, ma le sue lettere puerili e sgrammaticate non dicevano niente. Alla notizia che si era sposato mi meravigliai. Quando lo frequentavo, egli non mostrava interesse per nessuna delle donne che allora ci accadeva

5 d'avvicinare. La sua singolare bellezza suscitava ${ }^{1}$ l'amore in molte donne, ma egli disprezzava e scherniva ${ }^{2}$ crudelmente le ragazze che si erano innamorate di lui. Gli altri giovani nostri coetanei gli mostravano poca simpatia, ed io ero il suo solo amico.

Cinque anni circa dopo il suo matrimonio ricevetti una lettera di lui

10 che mi chiedeva di venirlo a raggiungere in una città balneare ${ }^{3}$, dove egli ora si trovava con la moglie e il bambino. [...]

Giunsi a mezzogiorno e scesi in una stazione calda, riverniciata di fresco e deserta. Walter mi aspettava addossato ${ }^{4}$ al muro, con le mani in tasca. Non era per nulla mutato. Portava un paio di pantaloni di tela $\mathrm{a}^{5}$ una

15 canottiera $^{6}$ bianca, con le maniche corte ed aperta sul collo. Sul suo viso

\footnotetext{
${ }^{1}$ suscitava : faceva nascere.

${ }^{2}$ scherniva: prendeva in giro, derideva.

${ }^{3}$ balneare: di mare, dove si fanno i bagni.

4 addossato: appoggiato.

5 tela : tipo particolare di tessuto, di lino o cotone.

${ }^{6}$ canottiera : maglietta, generalmente di lana o cotone, che si porta direttamente sulla pelle.
} 
grande, dorato dal sole, apparve un sorriso, ed egli mi venne incontro indolente $^{7}$ e mi porse la mano. [...]

Incontrammo Vilma che tornava dal bagno col bambino. Vidi una donna alta, un po' grossa, coi capelli neri ancora umidi e delle tracce di sabbia sul viso. Portava un abito da sole a quadretti che le lasciava le ginocchia scoperte, e aveva in mano un cappello di paglia ${ }^{8}$ intrecciata e una borsa di telacerata ${ }^{9}$ rossa. Il bambino mi parve piccolissimo, ma dissero che aveva quattro anni. Era un bimbetto magrolino, pallido, bello, con folti riccioli ${ }^{10}$ biondi che gli arrivavano alle spalle.

Abitavano un villino ${ }^{11}$ a due piani, davanti alla spiaggia. Mi era stata preparata una stanza al piano superiore, che dava non sul mare, ma sulla campagna. In tutta la casa c'era penombra e un buon odore fresco di legno e di pesche gialle. Si pranzava nella veranda ${ }^{12}:$ le tende di grossa tela color ruggine $^{13}$, mosse dal vento, si scostavano e lasciavano vedere il mare d'un azzurro splendente, il cielo e la spiaggia coi capanni ${ }^{14}$ dipinti a colori vivaci. $[\ldots]$

Mi alzavo molto presto alla mattina e mi affacciavo alla finestra : vedevo l'orto con la verde insalata rugiadosa e i fiori rossi e gialli, la vasta distesa $^{15}$ dei campi e i monti lontani velati d'un leggero vapore. Scendevo.

\footnotetext{
${ }^{7}$ indolente: con passo lento e pigro.

${ }^{8}$ paglia: materiale derivato da piante erbacee, usato soprattutto per fare borse e cappelli estivi.

${ }^{9}$ telacerata: di solito, tela cerata ; tela resa impermeabile con uno strato di vernice o gomma.

10 riccioli: capelli non lisci, a forma di anelli.

11 villino : piccola casa con giardino, di solito in campagna o al mare.

12 veranda: balcone spesso coperto con un tetto o chiuso da tende o vetrate.

${ }^{13}$ ruggine : bruno-rossastro, tipico dello strato che si forma sul ferro per umidità.

${ }^{14}$ capanni : piccole costruzioni, in genere di legno, che servono come spogliatoi sulle spiagge.

15 distesa: superficie piana molto grande.
} 
35 La spiaggia era ancora quasi deserta e la rena ${ }^{16}$, non ancora toccata dal sole, era umida e fredda. Vedevo Walter - egli si alzava ancor prima di me - uscire dall' acqua e venirmi incontro camminando col suo passo molle ${ }^{17}$ e leggero. [...] Un'americana di grande famiglia che aveva il capanno poco lontano dal nostro s'era innamorata di lui, e se lo vedeva solo s'avvicinava e gli vo-

40 leva parlare. Egli le rispondeva in modo poco gentile e se ne andava. L'aveva soprannominata "il pappagallo ${ }^{18}$ ". Dava soprannomi a tutti, e Vrasti era per lui ora "il vecchio pulcinella" ${ }^{19}$ " ora "il dottor Tartaglia" 20 ". Diceva quei soprannomi al bambino e lo faceva ridere.

Vilma e il bambino venivano sulla spiaggia molto tardi. Walter 45 prendeva il bambino in collo e lo portava nell'acqua, facendolo ridere e gridare di paura. Il piccolo aveva per lui un amore frenetico e vedevo che Vilma ne era gelosa.

$$
[\ldots]
$$

Passavo la maggior parte del giorno a vagabondare per la campagna con Walter.

Nelle nostre interminabili passeggiate egli stava quasi sempre in silenzio. Guardavamo il tramonto sdraiati su una roccia che scendeva a picco sul mare, in una vegetazione selvaggia di fichidindia ${ }^{21}$ e di palme. Che cosa fossero stati per Walter quegli anni in cui eravamo stati lontani, che

\footnotetext{
16 rena: sabbia.

${ }^{17}$ molle : senza forza.

${ }^{18}$ pappagallo : pappagalli da spiaggia in genere sono gli uomini che sulle spiagge vanno alla ricerca di donne con cui intrecciare una relazione.

${ }^{19}$ pulcinella : Pulcinella è una maschera napoletana; persona che cambia spesso opinione.

${ }^{20}$ il dottor Tartaglia : una maschera napoletana della commedia dell'arte, chiamata così a causa della caratteristica della balbuzie. Tartagliare è pronunciare in modo difettoso o incomprensibile.

${ }^{21}$ fichidindia: piante grasse con foglie trasformate in spine che producono un frutto dolce e saporito, anch'esso coperto di spine.
} 
55 cosa avesse fatto, creduto, sperato, io lo ignoravo, ma sapevo che ogni domanda sarebbe stata inutile. Lui stesso non mi rivolgeva alcuna domanda, e sapevo che non avrebbe preso alcun interesse a quello che di me gli avrei potuto dire. Tale mancanza d'interesse che in un altro m'avrebbe avvilito ${ }^{22}$, in lui mi appariva del tutto naturale, ovvia, non mi faceva soffrire. Capivo,

60 meglio di quanto non avessi fatto in passato, che egli era diverso ed avulso ${ }^{23}$ dagli altri esseri umani, e per questo ogni suo rapporto con gli altri prendeva una forma strana, inesplicabile ${ }^{24}$ ed offensiva per tutti ma non per me. Era come una grande pianta isolata. Il vento che soffia nelle sue fronde $\mathrm{e}^{25}$ e la terra che nutre le sue radici fanno parte della sua vita, non altro. Così io sen-

65 tivo che le gioie e i dolori di Walter non gli venivano dai suoi simili, ma da cause incomprensibili e sconosciute a noi, come la terra o il vento.

(da NATALIA GINZBURG, Casa al mare, in Opere, vol. I, Milano, Mondadori, 1986)

${ }_{22}^{2}$ avvilito : mortificato, depresso.

${ }^{23}$ avulso: distaccato.

${ }^{24}$ inesplicabile: inspiegabile.

25 fronde : i rami e le foglie. 
1. Vero o Falso?

\section{F}

a. Il narratore ed il suo amico Walter si scrivevano regolarmente per tenersi informati sulle vicende della loro vita.

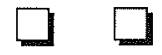

b. Walter era un tipo che non apprezzava le donne che mostravano interesse nei suoi confronti.

c. Nel villino c'era troppo buio.

d. Il narratore era sempre il primo ad alzarsi e a scendere sulla spiaggia.
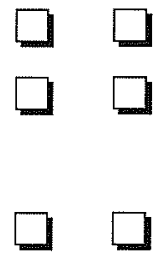

e. C'era armonia tra i membri della famiglia di Walter.

f. Nonostante che i due amici non si vedessero da tanto tempo, non si tempestavano di domande. $\square \square$

2. Trova nel testo parole o frasi che hanno il significato di:

a. proprie di chi ha una mentalità infantile

b. che hanno la stessa età

c. ridipinta da poco

d. bagnata dall'umidità dell'aria mattutina

e. cabina piuttosto grande per bagnanti

f. nome di una maschera napoletana della commedia dell'arte che rappresenta il tipo della persona ignorante e pettegola

g. gli voleva bene in modo pazzesco 
h. fare delle passeggiate senza una meta precisa

i. che discendeva verticalmente

1. distaccato dalle persone

3. Combina i seguenti modi dire con i relativi significati.

a. andare per mare ondata violenta

b. lupo di mare al largo, lontano dalla costa

c. essere in alto mare mare in burrasca

d. in mare aperto stretto, tra due terre

e. cercare per terra e per mare marinaio esperto

f. mare forza otto navigare

g. colpo di mare fare cose inutili

h. promettere mari e monti cosa di poco conto

i. braccio di mare fare promesse eccessive

I. una goccia nel mare tentare insistentemente e ovunque di trovare qualcosa o qualcuno

m. portare acqua al mare essere lontano dalla soluzione o dalla conclusione di qualcosa

4. Trasforma i verbi dal passato remoto al passato prossimo.

a. Cinque anni circa dopo il suo matrimonio ricevetti una lettera. (r.9)

b. Giunsi a mezzogiorno e scesi in una stazione calda, riverniciata di fresco e deserta. (r.12-13) 
c. Sul suo viso grande, dorato dal sole, apparve un sorriso, ed egli mi venne incontro indolente e mi porse la mano. (r.15-17)

d. Incontrammo Wilma che tornava dal bagno col bambino. (r. 18)

e. Vidi una donna alta. (r.18-19)

f. Il bambino mi parve piccolissimo, ma dissero che aveva quattro anni. (r.22-23)

5. Specifica se i pronomi sottolineati sono diretti, indirettio riflessivi, e spiega a chi o a che cosa fanno riferimento.

a. Alla notizia che si era sposato mi meravigliai. (r.2-3)

b. Mi chiedeva di venirlo a raggiungere in una città balneare, dove egli ora si trovava con la moglie e il bambino. (r.10-11)

c. Un'americana di grande famiglia che aveva il capanno poco lontano dal nostro s'era innamorata di lui, e se lo vedeva solo s'avvicinava e gli voleva parlare. (r.38-40)

d. Walter prendeva il bambino in collo e lo portava nell'acqua. (r.4445)

e. Che cosa fossero stati per Walter quegli anni in cui eravamo stati lontani, che cosa avesse fatto, creduto, sperato, io lo ignoravo. (r.53-55)

f. Lui stesso non mi rivolgeva alcuna domanda, e sapevo che non avrebbe preso alcun interesse a quello che di me gli avrei potuto dire. (r.56-58) 
6. Inserisci le preposizioni appropriate nel seguente brano tratto dallo stesso racconto.

Io vivevo allora ......... mia madre. Avevo un piccolo impiego che mi fruttava poco guadagno e .......... partire chiesi del denaro .......... mia madre. Ella mi accusò .......... sperpero e .......... poco riguardo lei e ci fu un leggero litigio. Ebbi .......... prestito il denaro uno zio e partii. Era una giornata calda ......... principio estate.

7. A riga 9 si viene a sapere che Walter ha scritto una lettera al narratore di questo brano. Immaginando di essere Walter, scrivi questa lettera, parlando:

- della moglie e del bambino

- della vostra casa al mare

- dell'invito che vuoi fare all'amico

8. Insieme ad un/a compagno/a di classe, crea un dialogo tra Walter ed il suo amico al momento del loro incontro alla stazione.

9. Spunti per la produzione orale e scritta.

a. Descrivi il tramonto visto da una spiaggia.

b. Dopo tanti anni, hai rivisto per caso un tuo compagno di classe. Narra il vostro incontro. 
c. Dove preferisci passare le tue vacanze, al mare, in montagna o in campagna?

d. Un viaggio indimenticabile.

e. L'arrivo inaspettato di un parente dall'estero.

f. Chi trova un amico trova un tesoro.

g. Riassumi e commenta il brano presentato.

\section{OLTRE IL BRANO}

Sinonimi : Fratelli sì ma non gemelli

\section{Nell'edilizia}

àndito : l'àndito, che è un semplice corridoio interno, che consente androne l'accesso a più stanze. L'androne costituisce il locale di atrio comunicazione tra il cortile interno di un edificio e la via. anticamera Più elegante l'atrio rispetto all'anticamera, comunque più ampio : si può parlare dell'atrio di una villa, di un palazzo, di un albergo, anche della stazione, di un ufficio pubblico, mentre l'anticamera è un locale con dimensioni limitate, dal quale si accede a un altro locale o al complesso delle camere di un normale appartamento.

***

balcone : il balcone c'è probabilmente anche nel nostro appartamenpoggiòlo to, verso strada, cortile o giardino : è di solito di limitate 
dimensioni. Anche il poggiòlo, che è diminutivo di "poggio" e come tale richiama piuttosto l'immagine di un balconcino. La loggia, invece, è più lunga e, nelle architetture di maggiore impegno, definisce l'apertura di tutto un edificio sulla facciata esterna, normalmente con archi sorretti da pilastri o colonne. Per indicare la loggia dei teatri, hanno fatto l'accrescitivo loggione (ma adesso si preferisce dire balconata, che naturalmente deriva da balcone).

水水

soffitta : nelle case urbane sembra migliore il termine soffitta, per

solaio indicare il vano tra l'ultimo piano e il tetto, che non solaio, abbaíno più idoneo per quel locale a palco dove si ripone a seccare mansarda il foraggio nelle case di campagna (il vocabolo deriva infatti dal latino solarium, "luogo esposto al sole"). Inoltre il solaio, come ben sanno i muratori, è il piano orizzontale che suddivide un edificio (è quindi il "pavimento" per il piano soprastante, "soffitto" per quello sottostante; inutile francesismo il vocabolo plafone). Quando diciamo abbaino ci riferiamo alle piccole costruzioni che fuoriescono dai tetti e che sono spesso abitate; il nome deriva da "abatino", cioè piccolo abate, perché il colore delle tegole di ardesia che in talune zone (in Francia, particolarmente) ricoprivano queste casucce sul tetto richiamò l'idea delle tonache degli abati. Se però vogliamo dare più prestigio a quelle dimore presso il tetto (e infatti i padroni di casa pretendono pigioni proporzionate a tale prestigio), le chiameremo piuttosto mansarde: nome che deriva da quello dell'architetto François Mansart, che nel secolo XVII le mise di moda nelle sue co- 
struzioni. Soprattutto in Francia, infatti, le mansarde sono la normale terminazione di molti antichi edifici.

colonna : la colonna è normalmente di marmo o granito e ha forma pilastro circolare. Il pilastro, in muratura, di pietra, mattoni o cepilone mento, è più spesso di forma quadrangolare. Anche il pilone lesena è in muratura, calcestruzzo, ma può essere anche di ferro, normalmente per sorreggere ponti o strade soprelevate. La lesena è anch'essa un pilastro, ma è sempre aderente al muro, e con lievissima sporgenza ; inoltre più che elemento di sostegno, la lesena è elemento decorativo, tanto che è spesso arricchita da cornici e da altri ornamenti.

米米

volta : la volta è la copertura, estesa all'intero soffitto, di un locaarcole le, di tutto un edificio; l'arco è l'elemento architettonico limitato tra due piedritti (colonne, eccetera) sul vano di una porta o di un ponte o altro.

cornicione : il cornicione è la fascia sporgente che circonda l'estremità gronda superiore di un edificio; la gronda è il prolungamento del tetto verso l'esterno, per riparo dalla pioggia e per apporvi il canale semicilindrico in lamiera per raccogliere l'acqua piovana e portarla a terra, detto appunto "grondaia".

水米

tégola : la tégola è genericamente ogni laterizio destinato alla cocoppo pertura esterna di un tetto; tanto vero che i tecnici usano émbrice distinguere le tégole curve dalle tégole piane. Le tégole curve son quelle di forma semicilindrica leggermente rastremata, cioè che vanno restringendosi verso il lato superiore, 
mentre quelle piano hanno forma piatta, rettangolare o trapezoidale. Naturalmente ci sono vari tipi ti tégole dell'una e dell'altra serie, ma i modelli tipici son sempre due : i coppi, che fan parte delle tégole curve, e gli émbrici, che fan parte delle tégole piane. I coppi li conosciamo tutti : semicilindrici, come s'è detto, sono d'uso antichissimo, tipicamente italiano. Gli émbrici sono d'uso più regionale, anche se conosciuti fin dagli antichi Romani. Spesso, nelle coperture dei tetti, i coppi si sposano agli émbrici : servono cioè a coprire la commessura tra un émbrice e l'altro.

(da Come parlare e scrivere meglio, Milano, a cura di A. GABRIELLI et alii, Selezione dal Reader's Digest, 1975, pp. 489-90)

\section{Opere di NATALIA GINZBURG (Palermo 1916 - Roma 1991)}

La strada che va in città, 1942 ; Tutti i nostri ieri, 1952 ; Valentino, 1957 ; Le voci della sera, 1961 ; Le piccole virtù, 1962 ; Lessico famigliare, 1963 ; Ti ho sposato per allegria, 1967 ; Mai devi domandarmi, 1970 ; Caro Michele, 1973 ; Paese di mare, 1973 ; Sagittario, 1975 ; La famiglia Manzoni, 1983 ; La città e la casa, 1984 ; L'intervista, 1988 ; Serena Cruz o la vera giustizia, 1990. 


\section{2 \\ L'UMBRIA}

L'Umbria si presenta a noi con aspetto tranquillo, senza colori drammatici. I più ${ }^{1}$ la guardano solamente dall'angolo della bellezza artistica e naturale. Gli stranieri ne hanno una nozione $\mathrm{e}^{2}$ molto semplice e molto gradevole : una fitta ${ }^{3}$ corona di città piccole e medie tutte stupende, fuse ${ }^{4}$ dal

5 bel paesaggio e dai ricordi francescani, con una popolazione dall'indole ${ }^{5}$ graziosa e dolce. Non vi è, ch'io sappia, recente scrittore italiano, che abbia portato l'Umbria alla coscienza del paese, come altri hanno fatto con le regioni dove i contrasti appaiono più vistosi. L'attenzione dei più è trattenuta dall'immagine convenzionale ${ }^{6}$, anche se in buona parte giusta, dell'Umbria 10 verde e azzurra, francescana e raffaellesca ${ }^{7}$.

Basta passare da Cortona, ancora in Toscana, a Perugia, per cogliere una profonda diversità d'animi e di paesaggi. La bellezza toscana, specialmente nell'arte, è più rigorosa ${ }^{8}$ e astratta, ed ha tra le sue muse ${ }^{9}$ la geometria.

${ }^{1}$ I più : la maggior parte delle persone.

${ }^{2}$ nozione : conoscenza.

${ }^{3}$ fitta : densa e compatta.

${ }_{5}^{4}$ fuse : legate con armonia.

5 indole : carattere.

${ }_{7}^{6}$ convenzionale : solita, usuale.

${ }^{7}$ raffaellesca: dalla forma caratteristica della pittura di Raffaello Sanzio (1483 1520).

${ }^{8}$ rigorosa: seria e severa.

${ }^{9}$ muse : fonti di ispirazione. Nella mitologia classica, le muse sono le nove figlie di Zeus e Mnemosine (personificazione della memoria), dedite al canto e alla danza sotto la guida del dio Apollo, considerate protettrici delle attività intellettuali e artistiche, invocate dai poeti per ottenere l'ispirazione. 
Quella umbra è morbida, più stemperata ${ }^{10}$ e più sfumata ${ }^{11}$, con una costellazione $^{12}$ di città in altura : o poste in vetta ${ }^{13}$, come Perugia e Todi, o sulle pendici ${ }^{14}$ di un monte, come Gubbio ed Assisi, o, come Orvieto, su un basamento di tufo ${ }^{15}$. Benché tutta la storia dell'arte abbia segnato l'Umbria, la nota dominante è data dalle architetture sacre, civili e militari del Medio Evo. Dall'alto si contemplano paesaggi come patinati ${ }^{16}$, conche ${ }^{17}$ d'un verde argenteo, colline che scendono lentamente a valle recando torri, campanili, basiliche, monasteri. Tramonti limpidi, di un rosso privo di eccessi, sfumano sulle rocche ${ }^{18}$ e sugli oliveti, tra suoni di campane e rondini. L'aria leggera dà un senso di euforia ${ }^{19}$ fisica. La popolazione passeggia sulle terraz$\mathrm{ze}^{20}$ che sovrastano ${ }^{21}$ il panorama. [...]

Assisi

[...] Assisi, in pietra grigia e rosa, è come l'hanno fatta secoli di francescanesimo, secoli di pietà, e secoli anche di estetismo ${ }^{22}$ devoto. Assisi

10 stemperata: delicata, dolce, meno forte.

${ }_{12}^{11}$ sfumata: tenue.

12 costellazione: raggruppamento, un insieme.

${ }_{13}^{13}$ poste in vetta : situate in cima, sulla sommità.

${ }_{14}$ pendici : lati o fianchi.

${ }^{15}$ tufo : roccia formata da un'aggregazione di sedimenti di origine vulcanica, frequente nelle regioni centrali e meridionali d'Italia, dove viene usata come materiale da costruzione.

${ }^{16}$ patinati : coperti da una velatura che li rende lisci o come ossidati.

17 conche : bacini, luoghi bassi fra i monti, valli strette.

${ }^{18}$ rocche : fortezze di grandi dimensioni costruite di solito in cima ad un monte o in un luogo elevato.

${ }^{19}$ euforia : un senso di benessere, caratterizzato da allegria e ottimismo.

${ }^{20}$ terrazze : ampie spianate a belvedere, con balaustrate.

${ }^{21}$ sovrastano: dominano da una certa altezza.

22 estetismo : atteggiamento di chi conferisce valore predominante a elementi di raffinatezza. 
è tutta aperta. Dal nodo di vie medievali a cui sovrastano la Rocca Maggiore

30 ed il monte, in cui la sera ci si arrampica tra le luminarie ${ }^{23}$, dalle numerose terrazze, si aprono panorami idillici ${ }^{24}$, talvolta subitanei ${ }^{25}$, sulla pianura sottostante ; risplendono alle finestre i garofani ed i gerani ; ed anche il visitatore meno devoto e curioso dell'arte, parte da Assisi sotto la suggestione ${ }^{26}$ di una fantasia di olivi, di cipressi, di bei tramonti, di piccoli giardini, di 35 muriccioli ${ }^{27}$ ornati di rose rampicanti, di rondini, di ricordi sacri, che si fondono insieme e, a ripensarvi, quasi sfumano nell'irrealtà. Assisi, come tante città dell'Umbria, ma forse in misura maggiore, dà la felicità che deriva dalla grazia e dalla perfezione di tutto quello su cui posano gli occhi, senza che nulla di stridente ${ }^{28}$ si intruda ${ }^{29}$ a interrompere la contemplazione ed a gua40 stare l'abbandono. [...] Francescana è l'impronta della città, benché al centro si innalzi un tempio di Minerva, romano ; benché anteriore a san Francesco sia il Duomo, dedicato a san Rufino, forse il monumento più insigne ${ }^{30}$ per suggestione architettonica. Ma le due basiliche sovrapposte di San Francesco cominciarono a sorgere alla morte del santo ; e così le chiese di 45 Santa Chiara e San Pietro. Nella scia ${ }^{31}$ della pietà francescana rientrano le opere dei secoli successivi, fino al grazioso Oratorio dei Pellegrini. Di San Francesco parlano tutti i dintorni; si scenda alla Porziuncola, si salga sul Subasio all'Eremo delle Carceri, ritiro rupestre ${ }^{32}$ in una selva oscura di lec-

\footnotetext{
${ }^{23}$ luminarie : serie di luci che illuminano luoghi pubblici.

${ }^{24}$ idillici : incantevoli, calmi e quieti.

${ }^{25}$ subitanei : improvvisi.

${ }^{26}$ suggestione : fascino che deriva da uno spettacolo naturale o da un'opera d'arte.

${ }^{27}$ muriccioli : muri bassi di cinta.

${ }^{28}$ stridente: sgradevole.

${ }^{29}$ si intruda: si intrometta.

${ }^{30}$ insigne : famoso.

${ }^{31}$ Nella scia : sulle orme.

32 rupestre : formato da rocce ripide e scoscese.
} 
$\mathrm{ci}^{33}$ e querce $^{34}$, o più brevemente si scenda al Convento di San Damiano, 50 ancora oggi solingo ${ }^{35}$ e povero, e guardante la valle. La sua ricchezza è il panorama. Qui, su una terrazza, parlai con un frate erudito ${ }^{36}$ della "porta del morto ${ }^{37 \%}$, che abbiamo trovato a Gubbio, ma che ad Assisi è quasi altrettanto frequente. Solo che in quest'aria dolce essa perde l'aspetto fantomatico ${ }^{38} \mathrm{e}$ superstizioso che ha nell'altra città. Mentre discorrevamo sulla terrazza, le

55 rondini turbinavano ${ }^{39}$ davanti a noi sopra lo sfondo del tramonto e salivano in vortici ${ }^{40}$, quasi assorbite in cielo da trombe d'aria ${ }^{41}$. Parevano preludere $\mathrm{ad}^{42}$ un'apparizione miracolosa.

L'immensa importanza di Assisi per la sua storia religiosa e artistica, di fronte a tutto il mondo non soltanto cristiano, fa sì che ogni altra questione

60 diventa in essa secondaria. Assisi è responsabile di fronte al mondo di restare com'è.

(da GUIDO PIOVENE, Viaggio in Italia, Milano, Baldini \& Castoldi, 1993 )

\footnotetext{
${ }^{33}$ lecci : alberi del tipo di quercia sempreverde che crescono nell'area mediterranea. ${ }^{34}$ querce : alberi, spesso sempreverdi, con foglie piccole e scure, il cui frutto è la ghianda, cibo caratteristico del maiale. ${ }^{35}$ solingo : solitario.

${ }^{36}$ erudito : istruito, dotto, colto.

${ }^{37}$ porta del morto : nell'immaginario popolare, la porta per far uscire i defunti. Nella realtà storica, si tratta della porta laterale rispetto al portone principale ; è piccola e rialzata, facilmente difendibile dall'assalto di eventuali briganti, ed era usata durante il Medioevo per entrare in casa di notte, senza aprire il portone.

${ }^{38}$ fantomatico : misterioso.

${ }^{39}$ turbinavano: si spostavano roteando rapidamente, alla maniera di un turbine, cioè vorticosamente.

${ }^{40}$ vortici : violenti movimenti rotatori.

${ }^{41}$ trombe d'aria : colonne d'aria in rapido e violento movimento di rotazione su sé stesse.

${ }^{42}$ preludere ad: preannunciare.
} 
1. Dalle quattro alternative, scegli le due giuste.

a. L'Umbria è una regione unica perché

(I) gli scrittori ne hanno parlato tanto

(II) oltre al bel paesaggio e ai ricordi francescani, la popolazione è molto accogliente

(III) il suo paesaggio è caratterizzato da forme dolci e armoniose

(IV) i turisti non vi trovano bellezze artistiche e naturali

b. La bellezza umbra si differenzia da quella toscana perché

(I) è più delicata e tenue

(II) è più geometrica e severa

(III) è contraddistinta da città situate in modo più attraente

(IV) è contraddistinta da città con una storia particolare

c. I colori del paesaggio umbro sono

(I) forti e contrastanti

(II) sbiaditi e sfocati

(III) senza eccessi

(IV) sfumati e delicati 
d. Assisi è una città aperta perché

(I) si possono ammirare dei bellissimi panorami

(II) le strade creano degli ampi spazi

(III) all'improvviso ci si trova a guardare dall'alto una vasta pianura

(IV) ci sono dei giardini pubblici molto grandi

e. Assisi suscita nel visitatore un sentimento di felicità perché offre

(I) una vita movimentata

(II) una visione armoniosa

(III) forti contrasti

(IV) spazio per la contemplazione

f. Assisi ha un'impronta francescana perché

(I) ci sono le due basiliche dedicate a Francesco

(II) le due basiliche sono le chiese più antiche della città

(III) al Santo si deve la costruzione del Duomo dedicato a san Rufino

(IV) nella città e nei dintorni ci sono luoghi che ricordano Francesco

g. Il Convento di San Damiano è famoso per

(I) la presenza della "porta del morto"

(II) la sua ubicazione

(III) le ricchezze che vi si trovano

(IV) il panorama che si può ammirare 
2. Indica con quale significato, nel testo di Piovene, sono usate le seguenti parole.

a. fitta (r.4) : (I) impenetrabile (II) folta (III) insistente

b. fuse (r.4) : (I) amalgamate (II) sciolte (III) legate bene

c. coscienza (r.7) : (I) consapevolezza (II) comprensione (III) responsabilità

d. cogliere (r.11) : (I) sorprendere (II) staccare (III) non lasciarsi sfuggire

e. segnato (r.17) : (I) caratterizzato (III) indicato (III) annotato

f. aperta (r.29) : (I) libera (II) panoramica (III) sincera

g. fantasia (r.34) : (I) invenzione (II) immaginazione (III) varietà incantevole

h. guastare (r.39-40) : (I) rovinare (II) corrompere (III) danneggiare i. opere (r.46) : (I) prodotti (II) attività (III) costruzioni

1. parlano : (r.47) : (I) informano (II) ricordano (III) discutono 
3. Scrivi due frasi in cui è presente ciascuna parola, ma con un significato differente.

ricordi (r.5) - nome / verbo

parte (r.9) - nome/verbo

nota (r.18) - aggettivo / nome

militari (r.18) - aggettivo / nome

suoni (r.22) - nome / verbo

rosa (r.27) - aggettivo/ nome

misura (r.37) - nome / verbo

abbandono (r.40) - nome / verbo

centro (r.40-41) - nome / verbo

morte (r.44) - aggettivo / nome

oscura (r.48) - aggettivo / verbo

4. Sostituisci i pronomi relativi sottolineati con altri aventi la stessa funzione.

a. che abbia portato l'Umbria alla coscienza del paese (r.6-7)

b. che scendono lentamente a valle (r.20)

c. che sovrastano il panorama (r.24)

d. a cui sovrastano la Rocca Maggiore ed il monte (r.29-30)

e. in cui la sera ci si arrampica tra le luminarie (r.30)

f. che si fondono insieme (r.35-36)

g. che deriva dalla grazia e dalla perfezione (r.37-38)

h. su cui posano gli occhi (r.38) 
5. Nel brano seguente tratto dallo stesso libro, sono stati tolti tutti $i$ segni d'interpunzione. Riempi i quadratini con i segni adatti, inserendo le maiuscole all'occorrenza.

Per le sue chiese e le sue vie $\square$ che conservano ancora l'impronta medievale $\square$ Orvieto rende felice il viaggiatore romantico $\square$ il Duomo $\square$ con la facciata dalle vaste pagine che Lorenzo Maitani ideò e scolpì $\square$ con i mosaici che risplendono nel tramonto $\square$ col rosone dell'Orcagna e gli affreschi del Signorelli $\square$ è cosa troppo celebre perché qui si possa parlarne $\square$ sorse per ricordare il miracolo di Bolsena $\square$ avvenuto nel $1263 \square$ quando un prete boemo celebrando la Messa vide stillare il sangue dall'Ostia consacrata $\square$ onde la festa del Corpus Domini $\square$ detta qui del Corporale $\square$ perché il sangue bagnò il corporale oggi racchiuso in un reliquario di metalli preziosi e smalti $\square$ scenderemo anche nel pozzo di San Patrizio $\square$ costruito da Antonio da Sangallo nel Cinquecento $\square$ che attinge 1'acqua in fondo a una doppia scala a chiocciola che trapana il tufo $\square$ vi scendiamo per trovarci in una nebbia luminosa e verdognola. 
6. Riempi gli spazi vuoti di questo brano tratto dalla stessa opera con preposizioni semplici o articolate.

Perugia è la maggiore città ............. Umbria e ne è anche il protipo. La sua via principale, corso Vannucci, si stende tra la bellissima piazza, in mezzo ............ quale sorge la Fontana Maggiore, le sue splendide sculture, e una terrazza che domina una vasta parte ............ Umbria. Vivace ed insieme teatrale, come spesso avviene ............. Italia, dove la strada è palcoscenico, il corso ............. Perugia è dunque ............. una fontana e un panorama, e vi si respira un'aria gaia, dolce ed euforica. A uno dei capi, oltre la Fontana Maggiore, sono: la cattedrale gotica . accanto la loggia quattrocentesca ............. Braccio Fortebraccio, ed il Palazzo Comunale, ............. grandi trifore, che porta il grifo ed il leone ............. una pietra tra il rosso e il rosa, forse il più insigne monumento ............. Perugia. Dall'altro capo si spalanca la terrazza, dove passeggia il popolo, specie ora del tramonto, sovrastando lo spazio ............. cui si disegnano alture che scendono verso il piano, macchie ............. città lontane, Foligno, Assisi, Nocera, Gualdo, e ............. fondo monti sfumati fino Amiata. 
7. Riscrivi le frasi seguenti: (a) sostituendo i verbi sottolineati con nomi che abbiano la stessa radice; (b) apportando i necessari cambiamenti; (c) mantenendo il significato di partenza (es. I più la guardano solamente dall'angolo della bellezza artistica e naturale (r.2-3) : Lo sguardo dei più si focalizza solamente sulla bellezza artistica e naturale).

a. Basta passare da Cortona, ancora in Toscana, a Perugia, per cogliere una profonda diversità d'animi e di paesaggi. (r.11-12)

b. Dall'alto si contemplano paesaggi come patinati. (r.19)

c. La popolazione passeggia sulle terrazze. (r.23)

d. Il visitatore meno devoto e curioso dell'arte, parte da Assisi sotto la suggestione di una fantasia di olivi, di cipressi [...]. (r.32-34)

e. In quest'aria dolce la "porta del morto" perde l'aspetto fantomatico. (r.53)

8. Nel brano seguente, tratto dalla stessa opera, sono stati inseriti alcuni errori. Correggili.

Il tartufo nero ci trova in molti luogi dell'Italia centrali. Norcia però è la loro capitale. Piace specialmente ai francesi, mentre gli italiani inclinano al tartufo bianco di Alba. Di Norcia lo si esportano anche in Francia, dove pure regna il tartufo nero di Périgord. Ha sapore meno violento di quel bianco piemontesi, anzi si distingue per la delicatezza, specie se ravvivato da uno vino vecchio. La cerca dei tartufi neri nei dintorni da Norcia ha qualche cose di magica che non si trova ad 
Alba. Si vanno coi cani e coi maiali, e anche da soli, osservando come gli aruspici il volo non dei uccelli ma delle mosche. La dove le mosche volteggiano, il tartufo si appiatta; per il più al centro d'una chierica di terra bruciata e chiusa in un cerchio rossastra.

9. Spunti per la produzione orale e scritta.

a. Hai ascoltato questo messaggio nella segreteria telefonica : "Ciao, sono Andrea. Ho una settimana di ferie e vorrei venire a trovarti. Posso stare da te ? Hai dei giorni liberi, così potremo andare un po' in giro ? Aspetto una tua risposta. A presto." Rispondi con una email.

b. Il tuo primo viaggio in un paese straniero.

c. Descrivi un panorama visto dall'alto.

d. Un/a turista ti chiede gentilmente delle informazioni sulla tua città. Spiegagli/le in breve :

- la storia della tua città

- i posti da visitare

- le manifestazioni più importanti

- eventuali gite ad altre città vicine. 


\section{OLTRE IL BRANO}

\section{Calendimaggio ad Assisi}

Dalle feste di primavera che si celebravano per le campagne o per le strade delle città dell'Europa occidentale, e dalle vicende di una vita cittadina assisana che ha conosciuto oltre a quei rituali di inizio maggio, anche aspre lotte tra due diverse fazioni, è nato nel 1927, e poi con nuova formula nel 1954, il Calendimaggio di Assisi. Il Calendimaggio dura tre giorni : il primo giovedì, venerdì e sabato di maggio. La festa ha la struttura di una sfida, e a contendersi l'ambito Palio sono le due parti in cui si divide per l'occasione la città : Parte de Sopra e Parte de Sotto. [...]

La mattina del primo giomo risuonano per le strade le parole del banditore che, preceduto da tamburi e chiarine, annuncia il ritorno della primavera e l'apertura della festa. Nel pomeriggio, dopo la benedizione dei vessilli di Parte de Sotto nella basilica di San Francesco e di Parte de Sopra nella cattedrale di San Rufino, la Piazza del Comune ospita la cerimonia di consegna delle chiavi della città dal Sindaco al Maestro di Campo. Un gesto simbolico con cui quest'ultimo assume pieni poteri ricevendo anche dalla parte risultata vincitrice l'anno precedente, il Palio. E la città si trasforma in un teatro all'aperto dove "scene", cortei, giochi, sfide canore saranno sottoposte al giudizio di esperti - musicologi, scrittori, registi - chiamati a giudicare. La gara prende avvio con le "scene", ricostruzione di un momento di vita quotidiana medievale che le due Parti allestiscono nei più bei luoghi, vicoli, scorci, della propria parte di città. Straordinaria è l'abilità di quanti si impegnano nel ricostruire mestieri ambienti e situazioni. 
Musici, danzatori, giullari, nobili e popolani, ognuno con la sua parte, ognuno con gesti e parole da dire e fare. L'intero quartiere viene trasformato in un angolo di Medioevo con taverne, botteghe, laboratori artigiani dove realmente si fabbrica carta, si tesse, si rilegano libri, si cuociono cibi. Si narra una storia, e per questa si scrivono i testi, di pura fantasia, oppure ispirati a fonti letterarie. [...] Il regolamento vuole che a dare avvio alle scene, al calar del tramonto, in un'atmosfera resa quindi ancor più suggestiva dalla luce tremolante e fioca delle fiaccole sia la Parte risultata perdente nell'edizione precedente. La sera dopo toccherà all'altra. Le "scene" si concludono sulla piazza del quartiere con una rappresentazione, sacra o profana, dove attori, cantanti, musici e danzatori non sono professionisti ma gli stessi abitanti. Arriva il giorno di Madonna Primavera. La regina della festa si elegge il venerdì pomeriggio, quando sul risultato di giochi e gare una delle due Part[i] se ne aggiudicherà l'elezione. Un bellissimo spettacolo di sbandieratori e tamburini fa da cornice al tiro alla fune, alla corsa della treggia, al tiro con la balestra e con l'arco.

$[\ldots]$

Di notte infine si svolgeranno le "scene" della Parte che ha vinto l'ultimo Calendimaggio. Si giunge al terzo giorno, il suono dei tamburi si fa sempre più intenso : è il giorno dei cortei, due per ogni Parte, uno di pomeriggio l'altro di sera. E anche qui lo spettacolo è grandioso. Centinaia di figuranti in bellissimi costumi si riversano sulla Piazza del Comune animandola con scenografie, coreografie, drammatizzazioni e spettacoli di rara suggestione e bellezza, di anno in anno diversi, ma sempre rivolti ad esaltare temi quali l'amore, l'armonia, la serenità, a celebrare la vita. [...] [Poi] è la volta dei cori che eseguiranno, dopo prove dure e durate mesi, il brano di sfida attin- 
to al repertorio dell'età tra Medioevo e Rinascimento, e identico per le due Parti. Dopo la gara canora, e dopo l'ultimo spettacolare corteo all'insegna del fuoco, è il momento della giuria : accompagnata dal Maestro di Campo si ritirerà nel Palazzo Comunale a redigere il verdetto.

La tensione si fa alta, il freddo della notte non scoraggia quanti sono lì, disposti ad aspettare le ore più piccole nella speranza di vedere il fazzoletto della propria Parte sventolare tra le mani del Maestro di Campo. Sì, perché è così che accade : sarà il Maestro di Campo a tornare in Piazza con in mano un cofanetto di legno e dopo aver pronunciate le parole di rito, comunicherà il vincitore ad una folla in assoluto e tesissimo silenzio estraendo uno dei fazzoletti che contiene : rosso oppure azzurro. Un gesto che fa esplodere la Piazza fra lacrime di gioia per una Parte, di amarezza per l'altra.

(da Calendimaggio di Assisi : musica, rito, storia e arte, Ensemble Micrologus, 1997, pp.11-18)

\section{Opere di GUIDO PIOVENE (Vicenza 1907 - Londra 1974)}

La vedova allegra, 1931; Lettere di una novizia, 1941; Gazzetta nera, 1943 ; Pietà contro pietà, 1946; I falsi redentori, 1949 ; De America, 1953 ; Viaggio in Italia, 1957 ; La coda di paglia, 1962 ; Le furie, 1964 ; Le stelle fredde, 1970. 
AUTORE IGNOTO. DEL SECOLO XIV

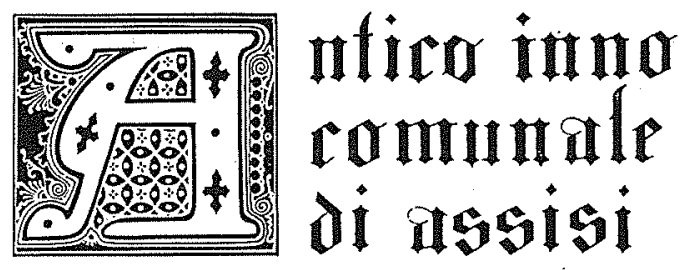

\section{IL COPRIFOCO}

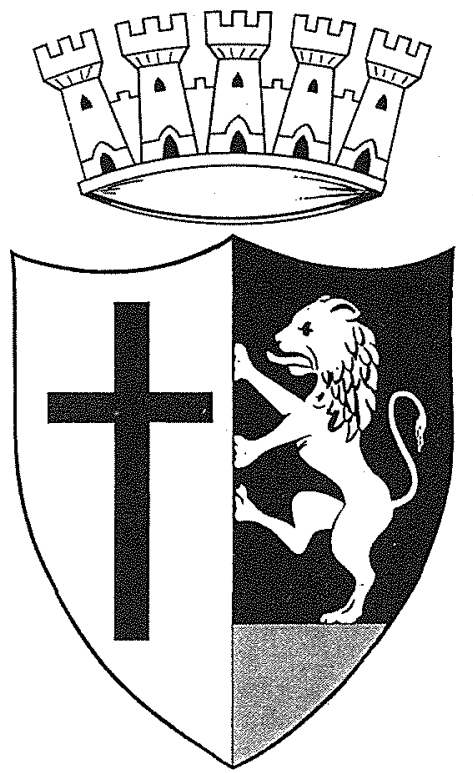

Trascrizione del

Adattamento ritmico del

M. FILIPPO GARAGNANI

G. Uff. Avv. ARNALDO FORTINI

\section{a ssisi rappella musionle s, rufino 1966}

* "Il Coprifoco" è eseguito dal coro I cantori di Assisi, direttore Padre Evangelista Nicolini, che ringraziamo sentitamente per la preziosa collaborazione. 


\section{IL COPRIFOCO}
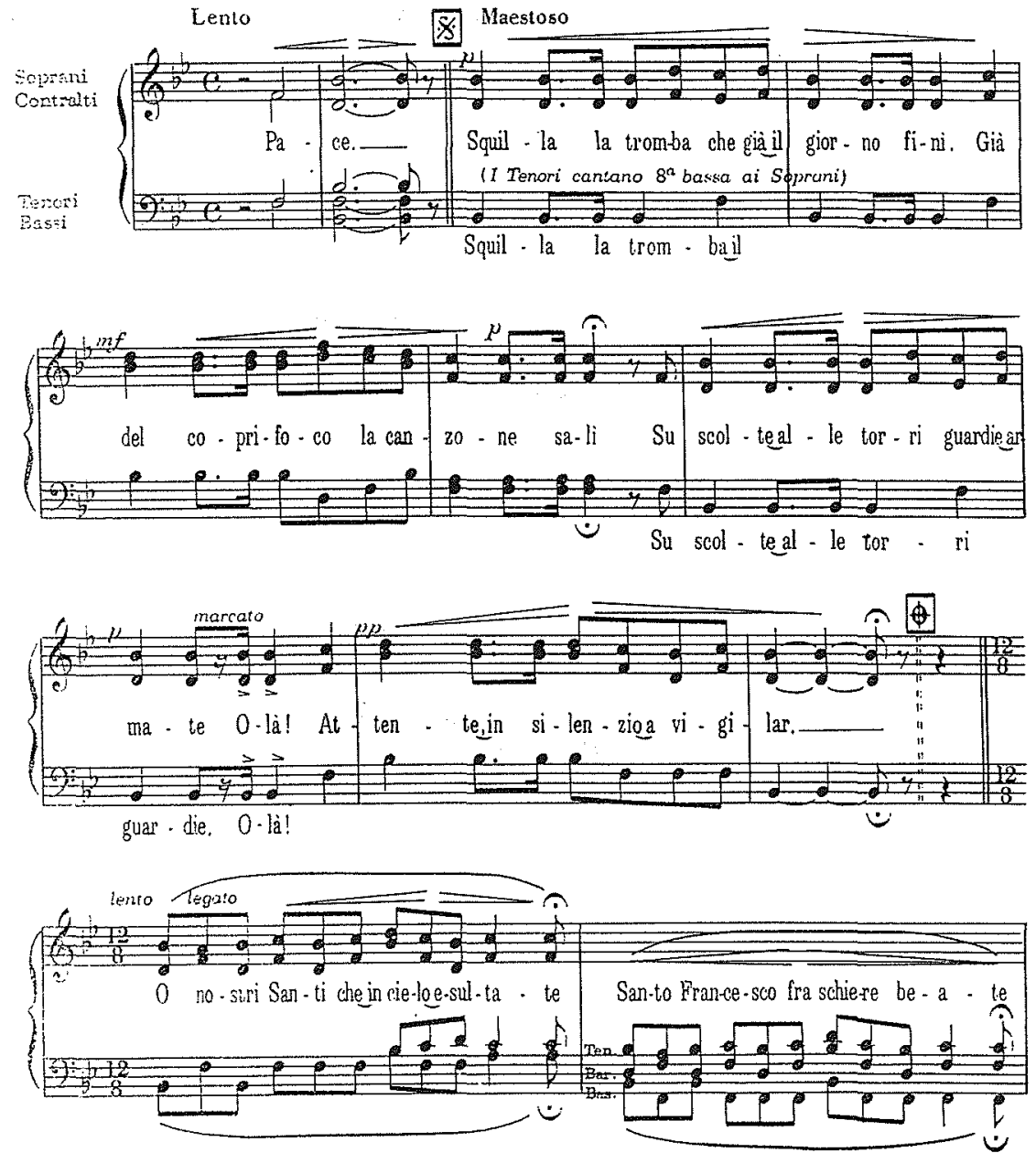

Linginale era a due sole roci costituite dalle due parti superiori, come inlatti viene tutitora suonato dagli Araldi del Corause di Assisi. La $3^{\text {j }}$ parte aggiunta dal traserittore complets l'armonizzazione nella riduzione per pianoforte e nella esecuzione a voci. 

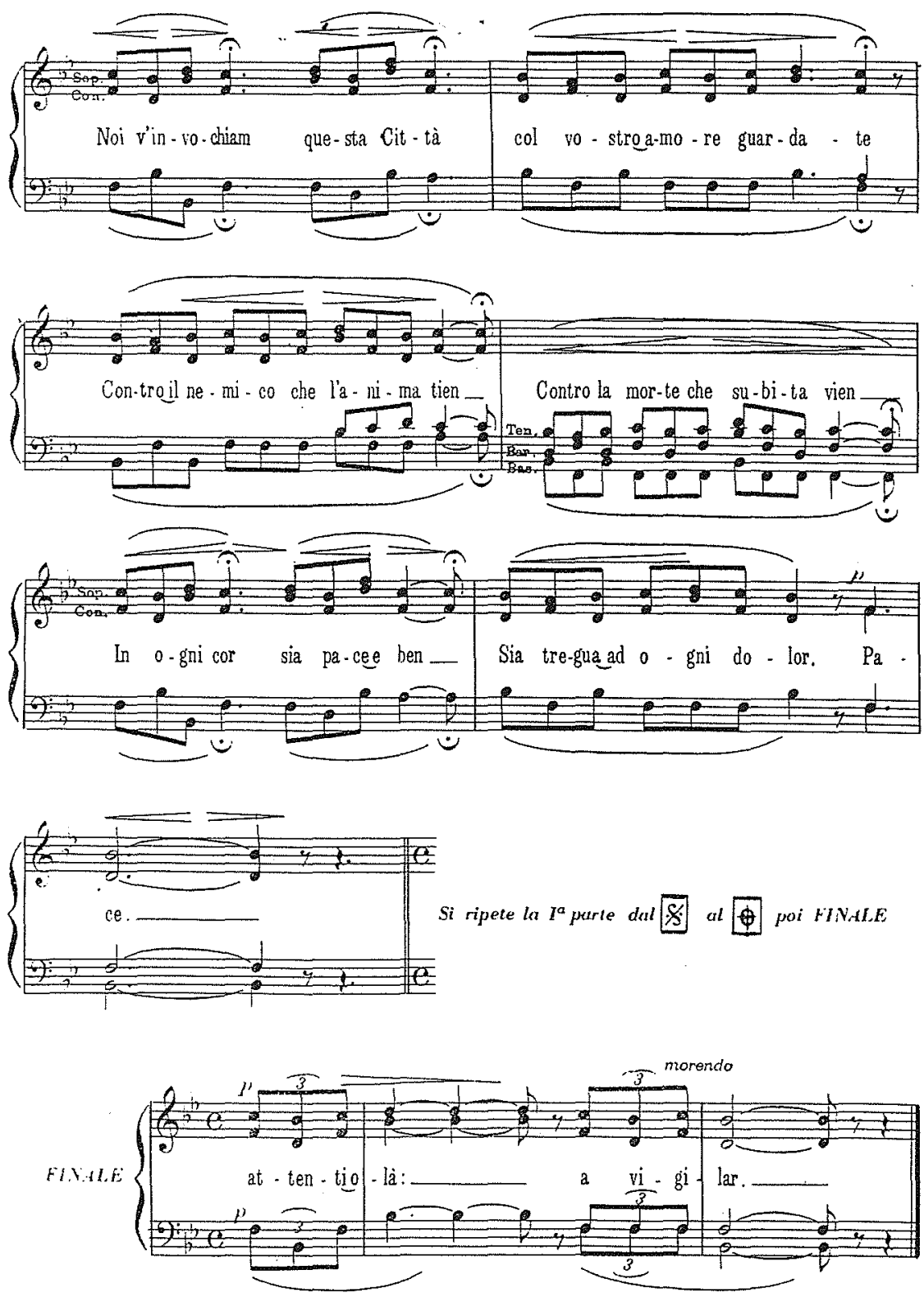


\section{3 \\ TRE VIE}

C'è a Trieste una via dove mi specchio ${ }^{1}$

nei lunghi giorni di chiusa tristezza :

si chiama Via del Lazzaretto ${ }^{2}$ Vecchio.

Tra case come ospizi ${ }^{3}$ antiche uguali,

ha una nota, una sola, d'allegrezza :

il mare in fondo alle sue laterali ${ }^{4}$.

Odorata di droghe $e^{5}$ e di catrame ${ }^{6}$

dai magazzini desolati a fronte,

fa commercio di reti, di cordame ${ }^{7}$

per le navi : un negozio ha per insegna

una bandiera ; nell'interno, volte

contro il passante, che raro le degna ${ }^{8}$

d'uno sguardo, coi volti esangui ${ }^{9}$ e proni ${ }^{10}$

\footnotetext{
${ }^{1}$ dove mi specchio : dove vedo più me stesso e la mia vita.

${ }^{2}$ Lazzaretto : ospedale dove un tempo venivano ricoverate le persone affette da malattie contagiose. In passato voleva semplicemente dire ospedale.

3 ospizi : luoghi in cui le persone anziane, che hanno bisogno di assistenza, vengono ricoverate.

${ }^{4}$ laterali : vie che si diramano da una via principale.

${ }^{5}$ droghe : in questo contesto, sostanze aromatiche vegetali usate per condire il cibo.

${ }^{6}$ catrame : liquido nero, denso e vischioso, usato, tra l'altro, per impermeabilizzare

le superfici legnose delle costruzioni navali e per coprire il manto stradale.

${ }^{7}$ cordame : insieme di corde. Le corde considerate come merce.

${ }^{8}$ degna: giudica meritevoli.

9 esangui : pallidi.

${ }^{10}$ proni : abbassati. 
sui colori di tutte le nazioni,

le lavoranti scontano la pena

della vita : innocenti prigioniere

cuciono tetre ${ }^{11}$ le allegre bandiere.

A Trieste ove son tristezze molte,

e bellezze di cielo e di contrada ${ }^{12}$,

c'è un' erta $^{13}$ che si chiama Via del Monte.

Incomincia con una sinagoga,

e termina ad un chiostro ${ }^{14}$; a mezza strada

ha una cappella; indi la nera ${ }^{15}$ foga $^{16}$

della vita scoprire puoi da un prato,

e il mare con le navi e il promontorio ${ }^{17}$,

e la folla e le tende del mercato.

Pure, a fianco dell'erta, $\mathrm{e}^{18}$ un camposanto

abbandonato, ove nessun mortorio

entra, non si sotterra più, per quanto

io mi ricordi : il vecchio cimitero

degli ebrei, così caro al mio pensiero,

se vi penso i miei vecchi, dopo tanto

${ }^{11}$ tetre : tristi.

${ }^{12}$ contrada: quartiere, rione ; a livello regionale significa strada di un centro abitato.

13 erta : salita.

${ }^{14}$ chiostro : cortile chiuso delimitato da portici all'interno di conventi, monasteri o abbazie ; convento.

15 nera: molto triste, oscura.

${ }^{16}$ foga : ritmo rapido con cui si agisce, l'impeto.

${ }^{17}$ promontorio : sporgenza montuosa della costa.

18 è : c’è. 
penare $^{19}$ e mercatare $^{20}$, là sepolti ${ }^{21}$, simili tutti d'animo e di volti.

Via del Monte è la via dei santi affetti, ma la via della gioia e dell'amore è sempre Via Domenico Rossetti. Questa verde contrada suburbana ${ }^{22}$, che perde dì per $\mathrm{di}^{23}$ del suo colore, che è sempre più città, meno campagna, serba $^{24}$ il fascino ancora dei suoi belli anni $^{25}$, delle sue prime ville sperse ${ }^{26}$, dei suoi radi filari ${ }^{27}$ d'alberelli.

Chi la passeggia in queste ultime sere d'estate, quando tutte sono aperte le finestre, e ciascuna è un belvedere,

${ }^{19}$ penare : faticare.

${ }^{20}$ mercatare : commerciare.

${ }^{21}$ sepolti : seppelliti, messi sotto terra.

22 suburbana: di una zona periferica della città.

${ }^{23}$ di per di : giorno per giorno.

${ }^{24}$ serba : mantiene.

${ }^{25}$ belli anni : fuori da questo contesto poetico, begli anni.

${ }^{26}$ sperse : isolate.

${ }^{27}$ filari : file, specialmente di alberi piantati. 
dove agucchiando ${ }^{28}$ o leggendo si aspetta,

rifiorirebbe all'antico piacere

di vivere, di amare lui, lui solo ;

e a più rosea salute il suo figliolo.

(da UMBERTO SABA, Trieste e una donna, in Antologia

del "Canzoniere", Torino, Einaudi, 1972)

1. Vero, Falso o Non Si Sa?

V F NSS

a. In Via del Lazzaretto Vecchio ci sono molti ospizi che creano un'atmosfera di tristezza.

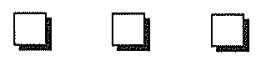

b. Il mare a lato di questa via costituisce il suo unico aspetto allegro.

c. Si vendono reti e vari tipi di corde solo ai pescatori triestini.

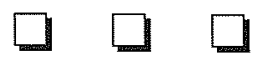

${ }_{28}^{28}$ agucchiando : lavorando con l'ago o con i ferri, senza particolare impegno.

${ }^{29}$ diletta: termine letterario, la persona amata. 


\section{F NSS}

d. Le operaie cuciono bandiere di varie nazioni dentro una bottega.

e. Ai piedi di una salita ripida chiamata Via del Monte, c'è un edificio per il culto della religione ebraica.

f. In cima alla via c'è un camposanto dove ormai non viene seppellito più nessuno.

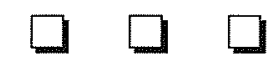

g. I genitori del poeta, e altri suoi familiari, giacciono nel vecchio cimitero degli ebrei.

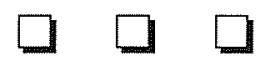

h. Via Domenico Rossetti è una via nel cuore della città.

i. Passeggiando per questa strada, la gente si sente sollevata e piena di speranze.
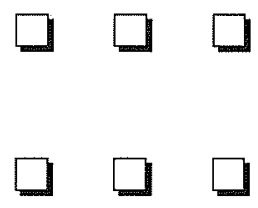

2. Indica con quale significato, nel testo di Saba, sono usate le seguenti parole :

a. chiusa (v.2) : (I) serrata, (II) introversa, (III) recintata

b. insegna (v.10) : (I) distintivo, (II) stemma, (III) cartello

c. tende (v.27)) : (I) sipari, (II) teli, (III) veli

d. mortorio (v.29) : (I) corteo funebre, (II) festa noiosa, (III) fastidio

e. vecchio (v.31) : (I) anziano, (II) antiquato, (III) antico

f. volti (v.35) : (I) caratteri, (II) aspetti, (III) facce

g. radi (v.45) : (I) larghi, (II) sparsi, (III) rari

h. rosea (v.53) : (I) di color rosa, (II) colorita, (III) florida 
3. Al v.4 c'è la similitudine "case come ospizi". Abbina le parole della prima colonna con i termini di paragone della seconda per formare similitudini di uso comune.
a. bagnato
come un toro
b. muto
come fratelli
c. sordo
come un pulcino
d. $\operatorname{cieco}$
come una Pasqua
e. beve
come un dio
f. mangia
come la luce del sole
g. si amano
come il fuoco
h. rosso
come una talpa
i. dorme
come una spugna
l. forte
come un lupo
m. contento
come una campana
n. bello
come un leone
o. chiaro
come un ghiro
p. coraggioso
come un pesce 
4. Al v.41, perde significa "cessa di avere". Accoppia le espressioni della prima colonna con il rispettivo significato dato nella seconda.

a. perdere il lavoro svenire

b. perdere terreno impazzire

c. perdere coscienza non essere più in rapporto

d. perdere il rispetto regredire

e. perdere $\mathrm{i}$ contatti non controllarsi più

f. perdere la pazienza lasciarsi sfuggire

g. perdere la ragione restare disoccupati

h. perdere la fede non dare/ricevere più stima

i. perdere un oggetto ... tralasciare volontariamente

1. perdere il treno ... smarrire

m. non perdere una non credere più sillaba

n. lasciar perdere subire una sconfitta

o. perdere la partita ... ascoltare con attenzione

5. Accoppia le parole prefissate della prima colonna con il rispettivo significato dato nella seconda.

a. sotterrare

b. atterrare dare in custodia

rivelare a qualcuno i propri problemi e segreti 

c. interrare
puntare una somma al gioco
d. suburbano
non fidarsi
e. conurbazione
introdurre nella terra
f. diffidare
interrompere
g. confidare
seppellire
h. affidare
agglomerazione costituita da una grande città e da centri periferici
i. sfidare periferico
l. scommettere invitare qualcuno a misurarsi in qualche prova
m. smettere toccare terra dopo un salto o un volo

6. In questa poesia vengono spesso utilizzati $i$ modi indefiniti. Rintraccia le forme del participio, del gerundio e dell'infinito.

7. Scegli dalla lista sottoelencata le parole o espressioni con la stessa funzione di quelle sottolineate.

a. una via dove mi specchio (v.1)

b. A Trieste ove son tristezze molte (v.19)

c. indi la nera foga della vita scoprire puoi (vv.24-25)

d. Pure, a fianco dell'erta, è un camposanto (v.28)

e. dopo tanto penare e mercatare (vv.33-34)

f. ma la via della gioia e dell'amore è sempre Via Domenico Rossetti (vv.38-39) 
g. quando tutte sono aperte le finestre (vv.47-48)

però, poi, in seguito a, nella quale, nel momento in cui, anche, dove.

8. In questa poesia si parla di tre vie: Via del Lazzaretto Vecchio, Via del Monte e Via Domenico Rossetti. Descrivi quello che secondo te caratterizza ognuna di queste vie.

9. Riscrivi in prosa questa poesia di Umberto Saba e spiega il suo significato.

\section{QUEST'ANNO...}

Quest'anno la partenza delle rondini mi stringerà, per un pensiero, il cuore.

Poi stornelli faranno alto clamore sugli alberi al ritrovo del viale XX Settembre. Poi al lungo male dell'inverno compagni avrò qui solo quel pensiero, e sui tetti il bruno passero.

Alla mia solitudine le rondini mancheranno, e ai miei dì tardi l'amore. 
10. Spunti per la produzione orale e scritta.

a. La strada dove abiti.

b. Quali sono i vantaggi che offre una casa in una zona periferica ?

c. Scrivi un dialogo tra due amici che si danno un appuntamento in piazza per passare la serata insieme.

d. Cosa trovi più interessante : leggere un libro di poesia o di prosa ?

e. Il tuo sogno nel cassetto.

f. Commenta un'altra poesia italiana che conosci bene.

\section{OLTRE IL BRANO}

\section{Sinonimi : Fratelli sì ma non gemelli}

\section{Per le vie della città}

via : via è generico : può essere larga, stretta, lunga, breve (può essere usato in senso traslato : la via del sapere, la via alla corso fede, la via migliore per ottenere uno scopo, ecc.). Il corso è più impegnativo : nel centro della città, solitamente contrada elegante, fiancheggiato da negozi. Anche la contrada è in città, e si addice piuttosto alle vie che hanno una tradizione storica (il vocabolo designa anche il rione, secondo la 
strada

viale

piazzale

piazza

largo

orto

parco

corte

cortile tradizione medievale). La strada normalmente esce dalla città (originariamente significava "via lastricata"). Il viale è ampio e alberato.

*水*

giardino : in latino hortus significa "giardino" (vocabolo, quest'ultimo, che entrò assai tardi nel nostro vocabolario, dal germanico attraverso il francese jardin, sostituendosi a brolo, che per più secoli aveva prevalso). Ormai noi diciamo orto per designare il terreno dove si coltivano gli erbaggi. Se un' amministrazione comunale istituisse un "orto pubblico" anziché un "giardino pubblico", si lamenterebbero a buon diritto i commercianti ortofrutticoli. Il parco è un giardino assai ampio, normalmente cintato, e può essere pubblico o privato. Da questo vocabolo abbiamo fatto derivare parcheggio (e il conseguente verbo parcheggiare) per il deposito delle nostre automobili.

: nel vocabolo corte c'è l'idea di qualcosa di più vasto rispetto al cortile, tanto che nel Medioevo significò l'insieme degli edifici intorno al castello del signore feudale, donde il significato di "sede del sovrano". In 
termini giudiziari e amministrativi : Corte d'assise, Corte d'appello, Suprema Corte (Cassazione), Corte

Costituzionale, Corte dei conti, eccetera. Le galline, invece, staranno più modestamente nel cortile, e anche i maiali e i conigli e gli altri animali definiti "da cortile".

米水米

muri $\quad:$ i muri sono quelli esterni delle case; le pareti sono le pareti costruzioni interne, che separano una stanza dall'altra. Le mura mura (plurale neutro alla latina di "muro") sono il complesso delle opere murarie, che in altri tempi si erigevano a protezione di una città, fortezza o castello. $* * *$

negozio : negozio, dal latino negotium, sembra più importante, si addice anche a una ditta commerciale. Un negoziante è bottega qualcosa di più che un bottegaio. Tuttavia la bottega, pur modestissima, dell'erbivendola potrà definirsi anch'essa negozio ; ma un grande negozio, per esempio, di libri, di articoli sportivi, o di abbigliamento, o di oreficeria, eccetera, non lo chiameremmo certamente bottega.

$* * *$

ponte : sono tre manufatti che scavalcano, superano qualche cosa, ma non sono la stessa cosa : hanno funzioni analoghe, ma non uguali. Il ponte è quello che scavalca un fiume, un corso d'acqua in genere : il ponte sull'Adige, sulla Senna, cavalcavia sul Tamigi. Il cavalcavia è sì simile al ponte, ma serve a scavalcare una via sottostante che si incrocia con quella 
soprastante ; si dice anche cavalcavia quell'arcata, quel passaggio soprelevato che mette in comunicazione due edifici scavalcando la via che li separa : in certe città medievali questi cavalcavia sono comunissimi. Infine il viadotto viadotto è, come struttura, simile a un ponte, ma non scavalca fiumi o strade, sì bene vallate, gole o avvallamenti di terreno per lo più stretti e profondi : sono infatti propriamente viadotti quelle maestose opere ardite che incontriamo frequentemente lungo le [...] autostrade.

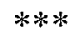

fiera

mercato

: la fiera (dal tardo latino ferria, propriamente "giorno festivo") è l'abituale convegno di commercianti, venditori e compratori, che si tiene in certi determinati giorni dell'anno, in particolari solennità che a volte coincidono con le feste sacre paesane ; vi si trattano affari in grande, e durano anche più giorni. Il mercato, invece, (derivato dal latino mercari, "commerciare, trafficare") è ugualmente un convegno di venditori e compratori, ma si svolge a volte tutti i giorni $\mathrm{e}$ in un luogo determinato, $\mathrm{o}$ anche più volte in una settimana o secondo date prestabilite, e tratta di merci varie o anche di un solo tipo di merci. Possiamo così avere il mercato del lunedì o del sabato, il mercato dei fiori, del pesce, della frutta, dei buoi, eccetera.

(da Come parlare e scrivere meglio, Milano, a cura di A.GABRIELLI et alii, Selezione dal Reader's Digest, 1975, pp. 73-4) 
Opere di UMBERTO SABA (Trieste 1883 - Gorizia 1957)

Canzoniere, 1921, 1948, 1951, 1961 ; Scorciatoie e raccontini, 1946 ; Storia e cronistoria del Canzoniere, 1948 ; Ernesto, postumo, 1975. 


\section{4 \\ LA FATTORIA DI RAMPINZERI}

Agosto 1860

"Gli alberi ! ci sono gli alberi !"

Il grido partito dalla prima delle carrozze percorse a ritroso ${ }^{1}$ la fila delle altre quattro pressoché ${ }^{2}$ invisibili nella nuvola di polvere bianca, e ad

5 ognuno degli sportelli volti sudati espressero una soddisfazione stanca.

Gli alberi, a dir vero, erano soltanto tre ed erano degli "eucaliptus"3 i più sbilenchi ${ }^{4}$ figli di Madre Natura ; ma erano anche i primi che si avvistassero da quando alle sei del mattino, la famiglia Salina ${ }^{5}$ aveva lasciato Bisacquino $^{6}$. Adesso erano le undici e per quelle cinque ore non si erano 10 viste che pigre groppe ${ }^{7}$ di colline avvampanti ${ }^{8}$ di giallo sotto il sole. [...] Mai un albero, mai una goccia d'acqua : sole e polverone'. All'interno delle vetture, chiuse appunto per quel sole e quel polverone, la temperatura aveva certamente raggiunto i cinquanta gradi. Quegli alberi assetati che si sbrac-

\footnotetext{
1 a ritroso: all'indietro.

${ }^{2}$ pressoché : quasi.

3 eucaliptus : eucalipti, genere di piante arboree con foglie aromatiche medicinali e fiori simili a piumini.

${ }^{4}$ sbilenchi: storti.

${ }^{5}$ Salina: dal nome di un'isola dell'arcipelago delle Eolie.

${ }^{6}$ Bisacquino : località in provincia di Palermo.

${ }^{7}$ groppe : vette collinari di forma arrotondata, come un dorso di quadrupedi.

8 avvampanti : accese.

${ }^{9}$ polverone : grande quantità di polvere sollevata, nuvola di polvere.
} 
ciavano $^{10}$ sul cielo sbiancato ${ }^{11}$ annunziavano parecchie cose : che si era 15 giunti a meno di due ore dal termine del viaggio; che si entrava nelle terre di casa Salina; che si poteva far colazione e forse anche lavarsi la faccia con l'acqua verminosa ${ }^{12}$ di un pozzo.

Dieci minuti dopo si era giunti alla fattoria ${ }^{13}$ di Rampinzeri ${ }^{14}$ : un enorme fabbricato, abitato soltanto durante un mese dell'anno da braccian$20 \mathrm{ti}^{15}$, muli ed altro bestiame che vi si radunava per il raccolto ${ }^{16}$. Sulla porta solidissima ma sfondata un Gattopardo ${ }^{17}$ di pietra danzava benché una sassata gli avesse stroncato proprio le gambe ; accanto al fabbricato un pozzo profondo, vigilato ${ }^{18}$ da quei tali eucaliptus, offriva muto i vari servizi dei quali era capace : sapeva far da piscina, da abbeveratoio, da carcere, da cimitero. Dissetava, propagava il tifo ${ }^{19}$, custodiva cristiani sequestrati, occultava $^{20}$ carogne ${ }^{21}$ di bestie e di uomini sinché si riducessero a levigati ${ }^{22}$ scheletri anonimi.

${ }^{10}$ si sbracciavano : come se i rami fossero braccia che si agitassero.

11 sbiancato : reso biancastro.

${ }^{12}$ verminosa : piena di animaletti invertebrati a corpo molle, allungato, privo di zampe, detti vermi.

${ }^{13}$ fattoria : azienda agricola.

${ }^{14}$ Rampinzeri : luogo immaginario ; un antico castello omonimo si trova in Sicilia, precisamente a Santa Ninfa, fra Segesta e Selinunte.

${ }_{15}^{15}$ braccianti : salariati agricoli non specializzati.

${ }^{16}$ raccolto : i frutti che si ottengono nell'operazione stagionale del cogliere e riunire prodotti agricoli giunti a maturazione.

${ }^{17}$ Gattopardo : mammifero carnivoro con corpo snello, testa lunga e stretta e pelliccia giallastra macchiata di nero; rappresenta lo stemma nobiliare della famiglia Salina. In seguito al successo del romanzo Il Gattopardo, si usa l'espressione filosofia del gattopardo e l'aggettivo gattopardesco per indicare il modo di pensare di coloro che sostengono che politicamente tutto deve cambiare nelle apparenze affinché nulla cambi nella sostanza.

18 vigilato : sorvegliato.

${ }^{19}$ tifo : infezione prodotta da batteri, caratterizzata da stato di torpore, che colpisce per lo più l'intestino tenue.

${ }^{20}$ occultava: nascondeva.

21 carogne : corpi di animali morti.

${ }^{22}$ levigati : lisci. 
Tutta la famiglia Salina discese dalle vetture. Il Principe, rallegrato dalla prospettiva di giungere presto alla sua Donnafugata ${ }^{23}$ prediletta $^{24}$, la

30 Principessa irritata ad un tempo ed inerte cui la serenità del marito, però, dava ristoro ${ }^{25}$; le ragazze stanche; i ragazzini eccitati dalla novità e che il caldo non aveva potuto domare ${ }^{26}$; mademoiselle Dombreuil, la governante francese, completamente disfatta e che memore ${ }^{27}$ degli anni passati in Algeria presso la famiglia del maresciallo Bugeaud andava gemendo: "Mon

35 Dieu, mon Dieu, c'est pire qu'en Afrique!"28 mentre si asciugava il nasino all'insù; Padre Pirrone cui l'iniziata lettura del Breviario ${ }^{29}$ aveva conciliato $^{30}$ un sonno che gli aveva fatto sembrare breve il tragitto ${ }^{31}$, e che era il più arzillo $^{32}$ di tutti; una cameriera e due servitori, gente di città irritata dagli aspetti inconsueti della campagna; e Bendicò che, precipitatosi fuori 40 dall'ultima vettura, inveiva ${ }^{33}$ contro $i$ suggerimenti funerei ${ }^{34}$ delle cornacchie che roteavano ${ }^{35}$ basse nella luce.

${ }^{23}$ Donnafugata : località che nel romanzo viene immaginata in provincia di Agrigento. In realtà, c'è una località che porta lo stesso nome, però si trova in provincia di Ragusa.

${ }^{24}$ prediletta : preferita, favorita.

25 ristoro: un senso di benessere.

${ }^{26}$ domare : addomesticare, fiaccare, soggiogare.

${ }^{27}$ memore : che conserva il ricordo di un'esperienza o di un fatto.

${ }^{28}$ Mon Dieu, mon Dieu, c'est pire qu'en Afrique! : Dio mio, Dio mio, è peggio che in Africa!

${ }^{29}$ Breviario : libro contenente le preghiere che i religiosi devono recitare durante la giornata.

${ }_{30}$ aveva conciliato : aveva facilitato.

${ }^{31}$ tragitto: strada percorsa.

32 arzillo : vivace (per lo più con riferimento a una persona da cui non si aspetta solitamente un portamento agile).

33 inveiva: mostrava, abbaiando, la sua disapprovazione.

${ }^{34}$ funerei : tristi, che danno l'idea della morte, lugubri.

${ }^{35}$ cornacchie che roteavano : grossi uccelli neri (simili ai corvi), con becco grosso e incurvato all'estremità, che giravano intorno. 
Tutti erano bianchi di polvere fin sulle ciglia, le labbra o le code ; nuvolette biancastre si alzavano attorno alle persone che giunte alla tappa ${ }^{36}$ si spolveravano l'un l'altra.

Tanto più brillava fra il sudiciume ${ }^{37}$ la correttezza elegante di Tancredi. Aveva viaggiato a cavallo e, giunto alla fattoria mezz'ora prima della carovana, aveva avuto il tempo di spolverarsi, ripulirsi e cambiare la cravatta bianca. Quando aveva tirato fuori l'acqua dal pozzo a molti usi si era guardato un momento nello specchio del secchio e si era trovato a posto, con quella benda nera sull'occhio destro che ormai serviva a ricordare più che a curare la ferita al sopracciglio buscata ${ }^{38}$ tre mesi fa ${ }^{39}$ ai combattimenti di Palermo; con quell'altro occhio azzurro che sembrava aver assunto l'incarico di esprimere la malizia anche di quello temporaneamente eclissato; col filetto ${ }^{40}$ scarlatto $^{41}$ al di sopra della cravatta che discretamente allu$\operatorname{deva}^{42}$ alla camicia rossa che aveva portato ${ }^{43}$. Aiutò la Principessa a scendere dalla vettura, spolverò con la manica la tuba ${ }^{44}$ dello zio, distribuì caramelle alle cugine e frizzi ${ }^{45}$ ai cuginetti, si genuflesse ${ }^{46}$ quasi dinanzi al Gesuita, ricambiò gli impeti ${ }^{47}$ passionali di Bendicò, consolò mademoiselle Dombreuil, prese in giro tutti, incantò tutti.

\footnotetext{
${ }^{36}$ tappa: sosta durante un viaggio.

${ }^{37}$ sudiciume : sporcizia.

${ }^{38}$ buscata: : subita.

${ }^{39}$ tre mesi fa: tre mesi prima.

${ }^{40}$ filetto : in questo contesto, piccola striscia di stoffa che si usa come ornamento.

${ }^{41}$ scarlatto: di color rosso vivo e splendente.

42 alludeva: faceva pensare.

43 camicia rossa ... portato : era stato garibaldino, cioè facente parte delle truppe, ricordate per la loro camicia rossa, comandate da Giuseppe Garibaldi (1807 -1882), uno degli artefici dell'Unità d'Italia.

44 tuba: cappello a cilindro.

45 frizzi : battute polemiche e pungenti.

46 si genuflesse: si inginocchiò.

47 impeti : scatti.
} 
I cocchieri ${ }^{48}$ facevano lentamente passeggiare in giro i cavalli per rinfrescarli prima dell'abbeverata ${ }^{49}$, i camerieri stendevano le tovaglie sulla paglia avanzata dalla trebbiatura ${ }^{50}$, nel rettangolino d'ombra proiettato dalla fattoria. Vicino al pozzo premuroso ${ }^{51}$ incominciò la colazione. Intorno ondeggiava la campagna funerea, gialla di stoppi ${ }^{52}$, nera di restucce ${ }^{53}$ brucia-

65 te; il lamento delle cicale riempiva il cielo; era come il rantolo ${ }^{54}$ della Sicilia $\operatorname{arsa}^{55}$ che alla fine di Agosto aspetta invano ${ }^{56}$ la pioggia.

(da GIUSEPPE TOMASI DI LAMPEDUSA, Il Gattopardo, Milano,

Feltrinelli, 1990)

\footnotetext{
48 cocchieri : guidatori di carrozze a cavalli.

49 abbeverata: dissetarsi degli animali.

${ }^{50}$ trebbiatura: operazione con la quale si separa la granella dei cereali dagli involucri della spiga, detti pula e dalla cosiddettta paglia fatta di steli di frumento.

51 premuroso : pieno di attenzioni, in senso ironico.

${ }^{52}$ stoppie : resti di steli e paglia che rimangono nel campo dopo la mietitura.

${ }^{53}$ restucce: steli e arbusti secchi rimasti che vengono bruciati per pulire il campo.

54 rantolo: respiro affannoso, tipico dei moribondi.

55 arsa : inaridita.

56 invano: inutilmente.
} 
1. Vero, Falso o Non Si Sa?

V F NSS

a. Il viaggio per Donnafugata era piacevole e divertente.

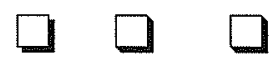

b. La campagna siciliana era arida e brulla a causa del gran caldo estivo.

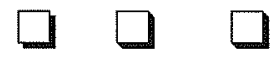

c. Le terre di casa Salina si estendevano per la maggior parte del percorso fatto dalla famiglia.

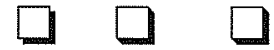

d. Il simbolo della famiglia, il Gattopardo, era stato colpito dal lancio di una pietra.

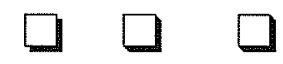

e. Il pozzo della fattoria serviva, fra l'altro, da cimitero, e l'acqua era così sporca da causare infezioni intestinali fatali.

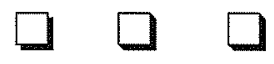

f. Tancredi teneva la benda nera sull'occhio destro perché era stato appena ferito al sopracciglio.

g. La famiglia Salina si fermò alla fattoria solo per fare colazione.

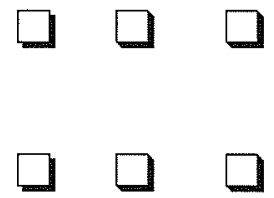


2. Trova nel testo parole o frasi che hanno i seguenti significati :

Dar.1 a r. 17

a. pendenti da una parte

b. ardenti per il gran caldo

Da r.18 a r.41

c. edificio di notevoli dimensioni

d. recipiente o vasca di notevoli dimensioni per far bere il bestiame

e. corpi in putrefazione, cioè in processo di decomposizione

f. procurava un senso di sollievo

g. allegro, vispo

h. volavano in senso circolare e non in alto

Da r.42 a r.59

i. gruppo di viaggiatori che si uniscono per attraversare regioni deserte, malsicure o disagevoli

I. oscurato perché coperto, ma solo per un periodo limitato di tempo m. cappello alto da uomo, ormai solo da cerimonia.

Da r.60 a r.66

n. residui di una coltura erbacea rimasti dopo il taglio o la mietitura

o. insetti di color nero-giallastro, comunissimi d'estate sugli alberi, che emettono un caratteristico verso. 
3. Indica con una $S$ i sinonimi e con una $C$ i contrari.
a. ritroso (r. 3)
in avanti
b. sudati (r.5)
madidi
c. assetati (r.13)
inariditi
d. sfondata (r.21)
intatta
e. levigati (r.26)
ruvidi
f. rallegrato (r.28)
afflitto
g. irritata (r.30)
esasperata
h. inerte (r.30)
zelante
i. eccitati (r.31)
agitati
1. inconsueti (r.39)
insoliti
m. passionali (r.58)
indifferenti
n. $\operatorname{arsa}($ r.66)
disseccata

4. Unisci le parti di proverbi contenute nella prima colonna con quelle relative poste nella seconda.
a. Chi non semina
è a metà dell'opera
b. A caval donato se son spine pungeranno
c. Chi ben comincia
si conosce 1'albero
d. Buon tempo e mal tempo
mare crespo
e. Cielo a pecorelle
e cervelli fini
f. Contadini, scarpe grosse
non si guarda in bocca
g. Dal frutto
non muore mai 
h. Chi semina vento

i. La mala erba

1. Se son rose fioriranno

m. Vento fresco

n. Una rondine non raccoglie acqua a catinelle non fa primavera raccoglie tempesta non dura tutto il tempo

5. Ricerca nel testo tutti $i$ verbi al trapassato prossimo.

6. Da questo brano, tratto dallo stesso romanzo sono stati omessi gli articoli determinativi ed indeterminativi. Inseriscili negli spazi vuoti.

........ Principe aveva sempre tenuto a che primo pranzo a Donnafugata avesse carattere solenne : figlioli sotto ........ quindici anni erano esclusi dalla tavola, venivano serviti vini francesi, vi era ......... poncio alla romana prima dell'arrosto; e domestici erano in cipria e polpe. Su di solo particolare transigeva : non si metteva in abito da sera per non imbarazzare ........ ospiti che, evidentemente, non ne possedevano. Quella sera, nel salone detto "di Leopoldo" ......... famiglia Salina aspettava .......... ultimi invitati. $\mathrm{Da}$ sotto ........ paralumi di merletto lumi a petrolio spandevano ......... gialla luce circoscritta; smisurati ritratti equestri dei Salina trapassati non erano che immagini imponenti e vaghe come ......... loro ricordo. 
7. Riempi gli spazi vuoti del brano, tratto dallo stesso romanzo, scegliendo una delle quattro proposte sottoelencate.

[...] Noi siciliani siamo stati avvezzi da una lunga, lunghissima egemonia di governanti che non erano (1) nostra religione, che non parlavano la nostra lingua, a spaccare i

(2) in quattro. Se non si faceva così non si scampava dagli esattori bizantini, dagli emiri berberi., dai viceré spagnoli. Adesso la piega è (3), siamo fatti così. Avevo detto "adesione", non avevo detto "partecipazione". In questi sei (4) mesi, da . (5) il vostro Garibaldi (6) piede a Marsala, troppe cose sono state fatte senza (7) perché adesso si possa chiedere ad un membro della vecchia (8) dirigente di svilupparle e portarle a (9). Adesso non voglio discutere se ciò che si è fatto è stato male o (10) ; per conto mio credo che molto .............. (11) male; ma voglio

(12) subito ciò che lei capirà da solo quando (13) un anno fra noi. In Sicilia non importa far male o far bene : il (14) che noi siciliani non perdoniamo mai è semplicemente quello di "fare". Siamo vecchi, Chevalley, vecchissimi. Sono venticinque secoli almeno che (15) sulle spalle il peso di magnifiche civiltà eterogenee, tutte venute da fuori, nessuna germogliata da noi stessi, nessuna a cui noi abbiamo dato il la. 
1 a) per la

b) con la

c) della

d) dalla

2 a) vetri

b) capelli

c) bicchieri

d) piatti

3 a) fatta

b) presa

c) completata

d) definita

4 a) ultimi

b) scorsi

c) passati

d) trascorsi

5 a) dove

b) come

c) quanto

d) quando

6 a) ha alzato

b) ha posto

c) ha preso

d) ha mosso

7 a) dirci

b) chiederci

c) consultarci

d) risponderci

8 a) classe

b) aula

c) folla

d) scuola

9 a) fine

b) traguardo

c) finale

d) compimento

10a) buono

b) bene

c) ottimo

d) meglio

11a) sia fatto

b) sia andato

c) sia compiuto

d) sia stato

12a) dirle

b) dirti

c) dirvi

d) $\operatorname{dirgli}$

13a) sarà passato $b$ ) sarà stato

c) sarà trascorso

d) sarà venuto

14a) male

b) vizio

c) peccato

d) problema

15a) portiamo

b) prendiamo

c) mettiamo

d) posiamo

8. Indica le righe delle sequenze sottospecificate, mettendole secondo l'ordine in cui vengono presentate.

a. Il comportamento di Tancredi

b. Descrizione della fattoria

c. Il caldo e l'arsura della campagna siciliana

d. La colazione sull'erba

e. Sentimenti di gioia alla vista degli alberi

f. L'aspetto di Tancredi 
g. L'aspetto predominante della morte

h. L'arrivo della carovana alla fattoria

9. Spunti per la produzione orale e scritta.

a. Preferisci viaggiare d'estate o in un'altra stagione ?

b. Un viaggio in macchina che non dimenticherai mai.

c. Scrivi una lettera ad un tuo parente, raccontandogli le difficoltà che hai dovuto affrontare durante un safari fotografico.

d. Era un bel giorno primaverile, quando i miei amici mi hanno chiamato/a per invitarmi ad un picnic...

e. La Sicilia : una regione ricca di bellezze artistiche e naturali.

f. Vedi il film Il Gattopardo e / o leggi il romanzo. Commenta.

g. Scrivi una breve biografia di Giuseppe Tomasi di Lampedusa e riporta tutti i dati seguenti :

- Luogo e anno di nascita : Palermo, 1896

- Di famiglia aristocratica (principi di Lampedusa)

- 1932 : matrimonio con la principessa Alessandra Wolff-Stomersee, studiosa di psicanalisi

- Appassionato lettore di libri storici e romanzi stranieri, soprattutto francesi

- Dal 1955 autore di opere narrative

- Romanzo famoso: Il Gattopardo (pubblicazione postuma, nel 1958) 
- 1963 : omonima riduzione cinematografica del regista Luchino Visconti, con Burt Lancaster, Claudia Cardinale e Alain Delon

- 1969 : ripubblicazione del romanzo

- Luogo e anno di morte : Roma, 1957.

\section{OLTRE IL BRANO}

\section{Il decalogo del mittente bene educato}

1. Se il destinatario è un parente o un amico, gli si rivolge con un caro, carissimo. Se è un superiore, una persona di riguardo o uno sconosciuto, scrive : Gentile signora, Chiarissimo professore, Egregio dottore. Sebbene logorato dall'uso, egregio contiene un lusinghiero apprezzamento, derivando da ex grege, fuori del gregge, ed è il contrario di gregario, colui che vive nel gregge.

2. Al papa si rivolgerà con il vocativo Santità, oppure Santo Padre, a un cardinale dirà Eminenza, a un vescovo Eccellenza, a un prelato Reverendo monsignore.

3. Dirà invece : Signor presidente della repubblica, Signor ministro, Signor prefetto, Signor generale, essendo stato abolito, nelle gerarchie laiche, il titolo di Eccellenza. Un titolo facilissimo da abolire. Da noi è stato soppresso parecchie volte, la prima fu con Garibaldi, che lo vietò appena sbarcato in Sicilia. Ma nonostante i divieti, esso sopravvive per lo struggente, intimo piacere che procura all'inferiore l'uso d'un titolo sonante, 
ampolloso, quasi lo facesse partecipare di rimbalzo all'onore, al potere, al carisma impliciti nel magico vocabolo. Ministro nell'immediato dopoguerra, Pietro Nenni stilò una circolare per abolire questo epiteto, retaggio d'una mentalità scarsamente democratica. Preparata la bozza, la diede ad un dattilografo del suo gabinetto, che ne batté alcune copie e poi le mise in una cartella sulla quale scrisse, con diligente grafia : "Alla firma di Sua Eccellenza il ministro".

4. Tra tutti i vocativi, signore resta il più elegante e dignitoso, per chi lo dà e per chi lo riceve. Purtroppo in Italia, l'abbiamo già visto, si preferisce usarlo come insulto o sfida :"Lei, signore, non sa chi sono io !"

5. Nelle lettere indirizzate a persona di riguardo, una vecchia consuetudine suggerisce di scrivere con la maiuscola i pronomi e gli aggettivi possessivi : Desidero ringraziarLa ; ricorderò sempre le Sue premure. A proposito della maiuscola, essa è d'obbligo : all'inizio d'una frase ; con i nomi propri, i cognomi, i soprannomi (Mario Rossi, Paolo Caliari detto il Veronese) ; libri, opere d'arte, giornali ( la Divina Commedia, la Cappella Sistina, la Gazzetta Ufficiale); toponimi (Via Margutta, Vicolo dell'Orso); feste e ricorrenze (il Natale, la Liberazione) ; fatti storici (il Quarantotto); simboli sacri (Dio, la Vergine) ; corpi celesti, intesi nel loro valore astronomico (la Terra gira intorno al Sole, però : sono caduto per terra, mia sorella ha preso troppo sole); enti, società, associazioni (la Corte di Cassazione, il Touring Club Italiano, la Rai, la Fiat); dopo i due punti che introducono un discorso diretto (chiese: "Mi presti centomila lire ?"). Per il re e il presidente della repubblica, non esistendo regole precise, l'uso della maiuscola si lascia alla fede, monarchica o repubblicana, dello scrivente.

6. Cari saluti è un non senso, osserva Giovanni Ansaldo nel Vero signore; i saluti sono cari a chi li riceve, non a chi li dà. Anche distinti saluti solleva 
perplessità. Esistono forse due tipi di saluti, quelli facili da confondere, e quelli che si distinguono a prima vista ? Altrettanto dicasi per sinceri auguri. L'augurio è sempre sincero. $\mathrm{Ci}$ mancherebbe altro che fosse falso, ipocrita. Se proprio desidera rafforzare auguri e saluti, il mittente premetterà affettuosi o fervidi o cordiali. Cordiali deriva dal latino cor, cuore. E un saluto fatto col cuore, dice tutto.

7. Una lettera si può scrivere anche a macchina. Non la firma, che deve essere sempre autografa.

8. Chi manda un invito per una conferenza, un congresso ecc. si guardi dallo scrivere "La S.V. è gentilmente invitata partecipare, ecc.". Quel gentilmente è una cafonaggine bella e buona, equivale a dire: "Io invito in maniera gentile la S.V. a partecipare" lasciando intendere che è anche possibile invitare in maniera sgarbata e villana.

9. Sbaglio peggiore commettono gli incauti che spostano il gentilmente e scrivono : "La S.V. è invitata a voler gentilmente partecipare". Un invito redatto in questi termini addirittura offende il destinatario, sospettandolo capace, qualora abbia la luna per traverso, di partecipare sì, ma villanamente.

10. Negli avvisi pubblici, nei bandi di concorso, i termini di scadenza per presentare una domanda, fare un ricorso o altro sono fissati con l'immancabile "entro e non oltre il [segue la data]". Il non oltre è una sciocca tautologia, spiegabile tramite Freud: infatti sottintende la non mai sopita vocazione italiana alla proroga, al rinvio.

(da C. MARCHI, Impariamo l'italiano, Milano, Rizzoli, 1984, pp.164-66) 
Opere di GIUSEPPE TOMASI DI LAMPEDUSA (Palermo 1896 Roma 1957)

Il Gattopardo, postumo, 1958 ; Racconti, postumi, 1961 ; Lezioni su Stendhal, postume, 1971. 


\section{5 \\ I "QUARTIERI"}

I "Quartieri", a Napoli, sono tutti i vicoli che da Toledo si dirigono sgroppando ${ }^{3}$ verso la città alta. Vi formicolano i gatti e la gente; incalcolabile è il loro contenuto di festini nuziali, di malattie ereditarie, di ladri, di strozzini ${ }^{4}$, di avvocati, di monache, di onesti artigiani, di case equivoche ${ }^{5}$, di

5 coltellate, di botteghini del lotto: Dio creò insomma i "Quartieri” per sentirvisi lodato e offeso il maggior numero di volte nel minore spazio possibile, e così facendo si legò le mani perché non di rado ${ }^{6}$ la sua collera, suscitata da qualche ignobile ruffiano $^{8}$, finiva per abbattersi erroneamente su un atti-

\footnotetext{
${ }^{1}$ Quartieri : nuclei di edifici o zone all'interno di un agglomerato urbano ; così venivano chiamati, originariamente, gli edifici costruiti dal governo vicereale spagnolo, usati come alloggio dai militari spagnoli. Attualmente il nome indica l'insieme di vicoli di questa zona della città.

${ }^{2}$ Toledo : toponomasticamente via Roma dopo l' Unità d'Italia, ma Toledo per i napoletani ; dal nome del miglior viceré di Napoli, don Pedro de Toledo.

${ }^{3}$ sgroppando : distendendosi, estendendosi.

${ }^{4}$ strozzini : usurai che prestano denaro ad interesse eccessivo, approfittando delle condizioni di necessità degli altri.

5 equivoche: malfamate.

${ }^{6}$ di rado: raramente.

7 ignobile : che manca di moralità.

8 ruffiano: chi, per guadagno, fa da intermediario in un incontro amoroso specialmente illecito.
} 
guo $^{9}$ falegname o ciabattino ${ }^{10}$, padre esemplare di cinque ragazze e organizzatore di pubbliche onoranze ${ }^{11}$ a San Vincenzo Ferreri ${ }^{12}$.

Il sole stesso percorre i "Quartieri" come può, ignorando interi edifici e concentrandosi furiosamente su uno scalino per arrostirvi qualche nero moccioso ${ }^{13}$ che sembra esservi stato abbandonato da anni; il sole dei "Quartieri" è al tempo stesso capriccioso e zelante ${ }^{14}$, trasforma in fulgide ${ }^{15}$ mitre $^{16}$ certe crepe ${ }^{17}$ di muro e lascia buie e fredde le finestre dei tisici ${ }^{18}$. Se poi il corteo funebre di un tisico, avviatosi dai "Quartieri" al Camposanto incrocia un carretto di erbivendolo, $i$ dolenti ${ }^{19}$ si sporgono dalle carrozze per acquistare un cespo di lattuga ${ }^{20}$ e irrorandolo ${ }^{21}$ di vere lacrime se lo mangiano al trotto lento dei cavalli sulla via di Poggioreale ${ }^{22}$.

Fu nei pressi del Teatro Nuovo: la tettoia ${ }^{23}$ dell'ingresso violentemente illuminata, i capannelli di spettatori curiosi delle donne che entrava-

9 attiguo : vicino.

10 ciabattino : chi ripara le scarpe.

11 onoranze : manifestazioni solenni per rendere onore a qualcuno, spesso alla memoria.

${ }^{12}$ San Vincenzo Ferreri : 1350-1419, predicò nella Bassa Francia, nell'Alta Italia e in Spagna, e fu al seguito di Papa Benedetto XIII.

13 moccioso : sporco di moccio, cioè di muco che cola dal naso.

${ }^{14}$ zelante : che agisce sollecitamente, senza indugio.

15 fulgide : che risplendono di viva luce.

${ }_{16}$ mitre : i copricapo di forma allungata che i cardinali e gli altri prelati portano nelle funzioni liturgiche solenni.

17 crepe: fessure, spaccature più o meno profonde.

${ }^{18}$ tisici : affetti da tubercolosi.

19 dolenti : persone in preda all'angoscia e al dispiacere.

${ }^{20}$ lattuga: un tipo di insalata verde.

21 irrorandolo: bagnandolo.

${ }^{22}$ Poggioreale: il nome del colle è da collegare alla villa reale, ampliata ed abbellita da Alfonso d'Aragona nel 1487. Attraversando via Poggioreale, si giunge al Cimitero Monumentale.

${ }^{23}$ tettoia : copertura a forma di tetto che serve a riparare uno spazio aperto sottostante. 
no, il fumo delle sigarette, i manifesti gibbosi ${ }^{24}$ per le intemperanze $e^{25}$ della colla e del vento di mare, l'odore di vecchio legno sfregato ${ }^{26}$ del botteghino,

25 il riso negli occhi della gente che pregustava l'irresistibile recitazione della compagnia Di Napoli - Della Rossa, e lì per terra, proprio dirimpetto ${ }^{27}$ al teatro, la botteguccia portatile, il negozietto di vimini ${ }^{28}$ di don Gennarino Aprile. $[\ldots]$

Calato il sipario uscivamo barcollando; fuori la notte si era impa30 dronita dei "Quartieri" ; diradatisi ${ }^{29}$ i passanti la botteguccia portatile di don Gennarino Aprile spiccava con le sue tre lampade ad acetilene ${ }^{30}$, con la sua pentola fumante, con la sua pila di piatti e di tazze contro l'escoriatissimo ${ }^{31}$ muro. Era un enorme cesto di vimini con tutto l'occorrente per allestire e spacciare $^{32}$ al defluente pubblico ${ }^{33}$ del Teatro Nuovo i peoci ${ }^{34}$ al succo di 35 limone, le alici ${ }^{35}$ fritte, ma specialmente il brodo di polipo ${ }^{36}$. Le dimensioni di questo polipo erano tali che sempre mi ricordavano la piovra ${ }^{37}$ descritta da Hugo in uno dei suoi fragorosi ${ }^{38}$ romanzi ; don Gennarino non si illudeva

\footnotetext{
${ }^{24}$ gibbosi : non lisci ma con sporgenze varie.

${ }_{25}^{25}$ per le intemperanze: per i danni causati da.

26 sfregato : consumato dall'uso.

27 dirimpetto : di fronte.

${ }^{28}$ vimini : rami sottili e molto flessibili di varie specie di salici, che dopo un opportuno trattamento vengono intrecciati per fabbricare oggetti domestici.

${ }^{29}$ diradatisi : diminuiti, e quindi diventati rari.

${ }^{30}$ acetilene : idrocarburo che si presenta come un gas incolore, di odore sgradevole, illuminante e infiammabile.

31 escoriatissimo : molto scrostato.

32 spacciare: vendere in quantità e /o con facilità.

33 al defluente pubblico : agli spettatori che escono da.

${ }^{34}$ peoci : cozze.

35 alici : pesci di mare di piccole dimensioni con corpo snello, dorso azzurro e ventre argenteo, chiamati anche acciughe.

${ }^{36}$ polipo : animale acquatico con corpo a forma di calice, munito di tentacoli.

${ }^{37}$ piovra : nome generico di molti molluschi di grandi dimensioni con lunghissimi tentacoli, muniti di ventose.

${ }^{38}$ fragorosi : celebri, strepitosi.
} 
di cuocerlo effettivamente, né in tutto né in parte : egli vi dava per due soldi una tazzina di bollente brodo del mostro (un cui solo tentacolo era bastato per riempire la pentola), ravvivata da un pizzico di pepe rosso e da un dado di polipo che equivaleva a circa un quarto della più piccola ventosa ${ }^{39}$. Dopo aver sorseggiato $^{40}$ l'infernale liquido, ed averlo sentito esplodere nello stomaco, ci si avviava verso casa masticando il frammento di polipo. Per ore esso sopravviveva intatto a ogni sforzo mascellare ${ }^{41}$, limitandosi ad emettere remoti e arguti ${ }^{42}$ odori marini che evocavano ${ }^{43}$ le miti ${ }^{44}$ acque di Santa Lucia $^{45}$, i repentini ${ }^{46}$ fremiti delle sabbie sommerse, le vele in deliquio ${ }^{47}$ sulla linea dell'orizzonte; andando a letto era opportuno sputare il pezzetto di polipo e non pensarci più, qualora non si volesse ricominciare a masticarlo l'indomani e per sempre. Desidero si sappia che il chewing-gum ha avuto un antenato al mio paese, presso il Teatro Nuovo ; quanto a don Gennarino Aprile, nessun dubbio che egli, nonostante la sua meschina ${ }^{48}$ statura, fosse un uomo completo e preciso. Quella sera io mi sentii brutalmente spinto da parte; un giovane e massiccio energumeno ${ }^{49}$ si fece largo in tal modo fino al canestro e ai piatti, si qualificò con un pauroso nomignolo ${ }^{50}$ e dichiarò

39 ventosa : organo in alcuni animali che serve per afferrare o aderire a qualche superficie.

${ }^{40}$ sorseggiato: bevuto lentamente a sorsi e assaporato.

${ }^{41}$ mascellare : relativo alle mascelle, le due parti che formano lo scheletro della faccia e in cui sono infissi i denti.

42 arguti: penetranti.

43 evocavano: richiamavano alla mente.

44 miti : quiete.

45 Santa Lucia : è il nome di un piccolo sobborgo, reso famoso da una celebre canzone napoletana, che si estende verso il mare.

46 repentini : improvvisi.

${ }^{47}$ vele in deliquio : barche a vela cullate dal mare.

48 meschina: bassa, inferiore alla media.

49 energumeno : chi è in preda all'ira e non sa dominarsi.

50 nomignolo : soprannome. 
55 che don Gennarino conosceva le ragioni della sua visita. Don Gennarino, concluse, doveva semplicemente rispondere sì o no.

"Un momento e sono a voi" ${ }^{51 "}$ rispose don Gennarino, interrompendo la vendita e cominciando a raccogliere piatti e tazzine.

(da GIUSEPPE MAROTTA, L'oro di Napoli, Milano, Rizzoli, 1996)

1. Scelta multipla. Due delle quattro alternative sono giuste e due sbagliate. Segna le due risposte giuste.

a. I "Quartieri” a Napoli sono

(I) zone di periferia sparse per la campagna

(II) delle strade intricate e sovrappopolate

(III) delle strade architettonicamente molto ordinate

(IV) piccole strade strette con le case ammassate

b. Nei "Quartieri", il sole

(I) arriva solo in alcune zone

(II) brucia tutti gli abitanti

51 a voi : da Lei. 
(III) non si vede perché gli edifici sono alti

(IV) non raggiunge quelli che veramente ne hanno bisogno

c. Vicino al Teatro Nuovo, si possono vedere

(I) le persone che raccontano barzellette e ridono

(II) $\mathrm{i}$ curiosi che guardano le donne che entrano in teatro

(III) le persone allegre perché immaginano il divertimento procurato dalla recita

(IV) gli uomini seduti per terra vicino al negozietto di don Gennarino Aprile

d. Quando finisce lo spettacolo, la gente

(I) si ferma da don Gennarino

(II) esce dal teatro rapidamente

(III) sembra stanca e cammina con passo insicuro

(IV) va direttamente a casa perché si è fatta notte

e. Dalla botteguccia di don Gennarino, la gente compra

(I) granite di limone

(II) cozze al limone

(III) brodo di polipo

(IV) zuppa di pesce

f. Una sera, alla botteguccia di don Gennarino, arriva

(I) un giovanotto educato e di bella presenza 
(II) un amico fraterno di don Gennarino

(III) un giovane dal fare sospettoso e dai modi violenti

(IV) un giovinastro che sembra in cerca di guai

2. Combina i gruppi di parole o espressioni con i relativi significati.

a. (I) botteguccia persona che gestisce la bottega

(II) botteghino cessare un'attività

(III) bottegaio piccola bottega

(IV) essere casa e bottega biglietteria

(V) chiudere bottega abitare molto vicino al posto di lavoro

o addirittura nello stesso edificio

b. (I) capanna

gruppo di persone che si riuniscono per

strada a discutere o ad ascoltare

(II) capanno

diminutivo di capanna

(III) capannuccia

grande capanna o tettoia chiusa, usata come deposito per attrezzi e prodotti agricoli. Fabbricato a un solo piano, utilizzato per attività industriali, artigianali, o come deposito merci.

(IV) capannello costruzione primitiva e provvisoria fatta di canne e paglia

(V) capannone cabina grande per bagnanti 
c. (I) carrozza

(II) carrozzabile

(III) carrozzaio

(IV) carrozzella

(V) carrozzina

d. (I) coltella

(II) coltellaccio

(III) coltellata

(IV) coltelleria

(V) coltelliera

e. (I) scalata

(II) scalo veicolo, a motore o spinto a mano, per

il trasporto di portatori di handicap, o per turisti, carrozza di piazza trainata da cavalli.

piccolo veicolo per portare a passeggio bambini molto piccoli

detto di strada che può essere percorsa da veicoli di qualsiasi tipo chi costruisce e ripara carrozze veicolo a quattro ruote, a uno o due cavalli, per il trasporto di persone

colpo di coltello che può causare ferita o morte tipo di coltello a lama larga astuccio, custodia per coltelli assortimento di coltelli. Negozio, fabbrica di coltelli coltello grosso e brutto gradino, cioè ognuno degli elementi che costituiscono i ripiani di una scala elenco schematico degli argomenti da sviluppare in un discorso. Elaborazione sommaria del soggetto di un film, con 
l'indicazione delle scene e degli ambienti

(II) scaletta scala di notevole ampiezza, per lo più esterna

(III) scalinata qualsiasi porto, aeroporto o stazione ferroviaria con impianti per l'arrivo e la partenza di merci e passeggeri

(IV) scalino ascesa verso il primato / potere o ascensione alpinistica

3. Accoppia i modi di dire contenuti nella $1^{\text {a }}$ colonna con $i$ significati relativi posti nella $2^{a}$.

a. passare una notte in bianco una persona di una famiglia / comunità considerata negativamente per comportamenti difformi dalla norma

b. mangiare in bianco preoccuparsi troppo

c. farsi venire $\mathrm{i}$ capelli bianchi usare caratteri di stampa i cui segni sono più spessi di quelli comuni $\mathrm{e}$ perciò appaiono più neri

d. mettere nero sul bianco arrabbiarsi moltissimo

e. essere nel libro nero fissare per iscritto un impegno o altro

f. una fumata nera trascorrere la notte senza dormire

g. la pecora nera della passare un giorno in cui tutto va famiglia storto 
h. essere proprio nero

i. scrivere in neretto

l. avere una giornata nera essere segnato come persona sgradita una votazione che dà risultato negativo attenersi a una dieta di carni e verdure bollite

4. Spiega a chi o a che cosa fanno riferimento i pronomi diretti o indiretti, e gli avverbi di luogo sottolineati.

a. vi formicolano $i$ gatti e la gente (r.2)

b. per arrostirvi qualche nero moccioso (r.12-13)

c. irrorandolo di vere lacrime (r.18)

d. mi ricordavano la piovra descritta da Hugo (r.36-37)

e. don Gennarino non si illudeva di cuocerlo (r.37-38)

f. egli vi dava per due soldi (r.38)

g. ed averlo sentito esplodere nello stomaco (r.42-43)

h. qualora non si volesse ricominciare a masticarlo l'indomani (r.4849)

5. Ricerca nel testo $i$ verbi al gerundio presente e trasformali dalla forma implicita in quella esplicita (es. : Andando a scuola, ho incontrato Eduardo / Mentre andavo a scuola, ho incontrato Eduardo). 
6. Sostituisci le congiunzioni sottolineate con altre congiunzioni o locuzioni congiuntive aventi funzione equivalente.
a. perché non di rado... (r.7)
b. come può (r.11)
c. se poi il corteo funebre di un tisico... (r.15-16)
d. ma specialmente il brodo di polipo (r.35)
e. qualora non si volesse ricominciare a masticarlo (r.48)

7. Crea in maniera autonoma un dialogo tra don Gennarino e il giovane energumeno.

8. Spunti per la produzione orale e scritta.

a. Descrivi un ambiente tipico della tua città.

b. Scrivi un dialogo tra una persona che è appena arrivata in una nuova città e una persona del luogo che fornisce delle informazioni e delle indicazioni importanti sulla città.

c. Al mercato.

d. Tradizioni e folklore del tuo Paese.

e. Suggerisci modi in cui i turisti possono scoprire veramente le caratteristiche della tua città.

f. Riassumi e commenta il testo con particolare attenzione agli aspetti satirico-umoristici. 


\section{OLTRE IL BRANO}

\section{I determinativi indiretti o complementi indiretti}

I determinativi indiretti nel corpo della frase [...] servono ad arricchire il messaggio che il parlante intende comunicare di tutte quelle informazioni, necessarie o accessorie, che sono sempre utili alla sua comprensione globale.

Le parole che adempiono a tale funzione sono collegate nel caso dei determinativi indiretti alla frase per mezzo di funzionali preposizioni. Quando essi sono collegati a un nome, si dicono complimenti del nome, quando sono collegati ad un verbo, si dicono complimenti del verbo, ma la funzione nei due casi è abbastanza simile.

L'importanza e la varietà di tali determinativi è grandissima : anche se essi non esercitano dal punto di vista logico una funzione primaria, come il soggetto e il predicato, bensì secondaria, tuttavia con la copia e la diversità delle informazioni che porgono, costituiscono dal punto di vista semantico uno strumento prezioso per precisare e comunicare le tante sfaccettature particolari in cui si articola globalmente un messaggio.

Quante preziose e svariate informazioni i complementi del nome e del verbo aggiungano alle strutture primarie delle frasi, lo si può constatare dalla lettura del seguente brano in cui sono in corsivo i vari determinativi indiretti : 
I "Quartieri", a Napoli, sono tutti i vicoli che da Toledo si dirigono sgroppando verso la città alta. Vi formicolano i gatti e la gente; incalcolabile è il loro contenuto di festini nuziali, di malattie ereditarie, di ladri, di strozzini, di avvocati, di monache, di onesti artigiani, di case equivoche, di coltellate, di botteghini del lotto: Dio creò insomma i "Quartieri" per sentirvisi lodato e offeso il maggior numero di volte nel minore spazio possibile, e così facendo si legò le mani perché non di rado la sua collera, suscitata da qualche ignobile ruffiano, finiva per abbattersi erroneamente su un attiguo falegname o ciabattino, padre esemplare di cinque ragazze e organizzatore di pubbliche onoranze a San Vincenzo Ferreri.

Il sole stesso percorre i "Quartieri" come può, ignorando interi edifici e concentrandosi furiosamente su uno scalino per arrostirvi qualche nero moccioso che sembra esservi stato abbandonato da anni; il sole dei "Quartieri" è al tempo stesso capriccioso e zelante, trasforma in fulgide mitre certe crepe di muro e lascia buie e fredde le finestre dei tisici. Se poi il corteo funebre di un tisico, avviatosi dai "Quartieri" al Camposanto incrocia un carretto di erbivendolo, i dolenti si sporgono dalle carrozze per acquistare un cespo di lattuga e irrorandolo di vere lacrime se lo mangiano al trotto lento dei cavalli sulla via di Poggioreale.

(Giuseppe Marotta).

Nel brano i determinativi indiretti che dipendono da un nome e quelli che dipendono da un verbo si distribuiscono equamente nelle due categorie dei complimenti del nome e complimenti del verbo. La maggior parte dei complimenti del nome sono introdotti dal funzionale preposizione $d i$. Li enumeriamo, ponendo tra parentesi il nome o il gruppo nominale che essi determinano :

(il loro contenuto) $d i$ festini nuziali, $d i$ malattie ereditarie, $d i$ ladri, $\quad d i$ strozzini, $d i$ avvocati, di monache, $d i$ onesti artigiani, $d i$ case equivoche, di coltellate, di botteghini del lotto.

(il sole) dei "Quartieri" 
(crepe) di muro

(le finestre) dei tisici

(il corteo funebre) di un tisico

(il carretto) di un erbivendolo

(un cespo) $d i$ lattuga

(al trotto lento) dei cavalli

(sulla via) di Poggioreale.

Nei complimenti suddetti il funzionale preposizione $d i$ introduce vari rapporti semantici : in genere il complimento del nome introdotto dalla preposizione $d i$ determina la specie o il genere a cui si riferisce il nome da essa determinato (un cespo di lattuga), ma tra gli altri esempi suddetti alcuni indicano anche altri rapporti :

la materia (esempio : il loro contenuto di festini ecc.);

l'appartenenza (esempio : il carretto di un erbivendolo) ;

il luogo (esempio : crepe di muro, via di Poggioreale ecc.).

(da C. ANNARATONE, M.T. ROSSI, Lingua e società, Bologna, Zanichelli, 1977, pp.330-32).

\section{Opere di GIUSEPPE MAROTTA (Napoli 1902 - 1963)}

L'oro di Napoli, 1947 ; San Gennaro non dice mai no, 1948 ; Gli alunni del sole, 1952 ; Il califfo Esposito, 1956 ; Gli alunni del tempo, 1960. 


\section{6 \\ VOGLIO LA TERRA}

[...] Un giorno, come poi si riseppe ${ }^{1}$, Berardo si presentò all'improvviso nello studio dell'avvocato; e dopo aver messo da parte la serva, che cercava di respingerlo adducendo ${ }^{2}$ l'assenza del padrone, lo cercò in tutte le stanze e lo trovò spaurito dietro le tendine di una finestra.

"Signor avvocato", gli disse con molta calma (anzi, com'egli ci raccontò, persino con rispetto) " $\mathrm{voi}^{3} \mathrm{mi}$ avete assicurato varie volte che io morirò in carcere ; non credete che sia venuto il giorno per andarci ?"

L'avvocato dovét capire che la sua vita era legata a un filo, eppure cercò di sorridere.

"Perché tanta fretta ?" egli balbettò ${ }^{5}$.

"L'occasione sarebbe buona" spiegò Berardo che aveva fretta. "È un'occasione unica per andarci con la coscienza tranquilla."

"Pensi sempre alla terra ?" aggiunse l'avvocato. "Perché non hai tentato un altro mestiere?"

\footnotetext{
${ }^{1}$ riseppe : si venne a sapere la notizia che circolò passando di bocca in bocca.

${ }^{2}$ adducendo : presentando come giustificazione.

${ }^{3}$ voi : Lei.

${ }^{4}$ dové : o dovette.

${ }^{5}$ balbettò : parlò articolando stentatamente le sillabe e ripetendole più volte, per paura.
} 

rispose Berardo. Poi aggiunse minaccioso: "io sono un cafone ${ }^{8}$ e voglio la terra."

"Ce n'è un'altra, di un altro genere" gli rispose allora don Circostanza. "Mi meraviglio che tu non ci abbia pensato da te stesso. Siediti e 20 ascoltami. Sopra Fontamara $^{9}$, nella contrada $^{10}$ dei Serpari, nell'avvallamento ${ }^{11}$ tra le rocce, si è formato un pascolo ${ }^{12}$ che appartiene al comune e che serve poco, forse a qualche capra, ma nulla più. È buona terra, e se la vuoi lavorare, per pochi soldi te la faccio assegnare dal comune."

Così don Circostanza si salvò. Berardo ricevette a poco prezzo il 25 campicello selvatico nella contrada dei Serpari, e di nuovo egli ebbe una sua terra. Ma per dissodarla ${ }^{13}$ furono fatiche bestiali che Berardo dovette affrontare al mattino presto e alla sera tardi, come pure nei giorni festivi, perché per comprare le sementi ${ }^{14}$, e per vivere con la madre, egli non poteva rinunziare a guadagnarsi la giornata come gli altri cafoni. Quando all'alba noi ${ }^{15}$ 30 mettevamo il basto ${ }^{16}$ sull'asino per andare al campo, vedevamo Berardo

\footnotetext{
${ }^{6}$ trote : pesci d'acqua dolce.

${ }^{7}$ passeri : uccelli molto comuni che hanno corpo grigio bruno, gola e ali striate di nero.

${ }^{8}$ cafone : contadino; si usa, per estensione, per una persona villana e maleducata.

${ }^{9}$ Fontamara: un paesino immaginario, ma con caratteristiche sociali reali, del bacino del Fucino, in Abruzzo. La vicenda viene ambientata all'inizio del periodo fascista (1922-43).

${ }^{10}$ contrada: zona o strada secondaria di luogo abitato. A Siena però, ciascuno dei quartieri in cui è divisa la città.

11 avvallamento : una parte del territorio bassa rispetto alle zone circostanti.

${ }_{13}^{12}$ pascolo : terreno erboso adatto per l'alimentazione del bestiame.

${ }^{13}$ dissodarla: renderla coltivabile.

${ }^{14}$ sementi : insieme di semi destinati alla semina.

15 noi : i cafoni di Fontamara.

${ }^{16}$ basto : rozza sella di legno, usata per cavalcare asini e muli o per assicuravi i carichi.
} 
scendere dalla montagna con la zappa ${ }^{17}$ sulla spalla e seguirci ; e quando la sera, dopo aver mangiato la minestra, noi rientravamo in casa per coricar$\mathrm{ci}^{18}$, vedevamo Berardo prendere la via della montagna.

"Tu ti ammazzi" gli dicevamo. "Non devi abusare della tua forza, ti 35 rovini."

"O la montagna ammazza me, o io ammazzo la montagna" rispondeva Berardo ridendo.

"Non parlare cosi" 1'ammoniva ${ }^{19}$ Michele Zompa. "Puoi agire come ti pare, ma non sfidare la montagna con le parole."

A Berardo però in fondo gli volevamo tutti bene. Aveva anche lui i suoi difetti, specialmente da ubriaco, ma era leale e sincero ed era stato assai sfortunato, e per questo, di buon cuore, gli auguravamo che potesse rifarsi ${ }^{20}$ la terra. Il giorno perciò che ci disse di aver seminato un sacco di granotur[c]o nella contrada dei Serpari (dove mai, a memoria d'uomo, era stato seminato), tutti ci rallegrammo con lui e bevemmo alla sua salute. "Ho domato ${ }^{21}$ la vecchia montagna", diceva Berardo ridendo. Forse ci rallegrammo troppo, forse ci rallegrammo affrettatamente ; forse, come temeva Michele, in quel modo sfidammo la montagna.

Quello che successe due mesi dopo, è risaputo. Benché i vecchi rac50 contassero già fatti simili, siccome ognuno crede soltanto a quello che vede coi propri occhi, quella è una data che noi non dimenticheremo facilmente. E certi fatti è meglio raccontarli in poche parole, perché a rifletterci sopra non serve. Piovve dunque per tre giorni, ma non in una quantità ecceziona-

\footnotetext{
${ }^{17}$ zappa : attrezzo agricolo manuale formato da una lama di ferro infilata in un manico di legno che serve a rompere le zolle o a fare solchi.

${ }^{18}$ coricarci : metterci a letto.

${ }^{19}$ ammoniva : consigliava con autorità.

${ }^{20}$ rifarsi : riprendersi.

21 domato : sottomesso.
} 
le ; la cima della montagna sopra Fontamara era avvolta da un nuvolone 55 nero che non lasciava capire nulla. E all'alba del terzo giorno venne giù dalla montagna, col fragore ${ }^{22}$ di un terremoto, in direzione della contrada dei Serpari, come se la montagna crollasse, un'enorme fiumana ${ }^{23} \mathrm{~d}$ 'acqua che portò via il campicello di Berardo, come un affamato vuota un piatto di minestra, scavando la terra fino alla roccia e disperdendo nella valle le piantine 60 verdi del granoturco. Al posto del campo coltivato rimase un'enorme fossa, una specie di cava ${ }^{24}$, una specie di cratere.

(da IGNAZIO SILONE, Fontamara, Milano, Mondadori, 1973)

1. Tra le quattro alternative proposte, due sono giuste e due sbagliate. Scegli le due giuste.

a. Berardo non riuscì a trovare subito l'avvocato perché

\footnotetext{
${ }^{22}$ fragore : rumore violento e intenso.

${ }^{23}$ fiumana: corrente come di un fiume in piena.

${ }^{24}$ cava: luogo scavato, allo scoperto, da dove si estraggono (si cavano) minerali o materiali da costruzione.
} 
(I) la serva cercò di bloccare Berardo.

(II) l'avvocato s'era nascosto dietro le tende.

(III) 1'appuntamento era più tardi

(IV) l'avvocato era uscito

b. L'avvocato cominciò a balbettare perché

(I) Berardo era un criminale

(II) Berardo aveva un tono minaccioso

(III) aveva preso la terra di Berardo

(IV) sentiva che la sua vita era in perciolo

c. Berardo voleva fare solo il contadino perché

(I) non gli piaceva fare il pescatore

(II) voleva stare in contatto con la natura siccome gli piacevano molto $\mathrm{i}$ passeri

(III) era molto attaccato alla terra

(IV) non si sarebbe mai sentito a suo agio facendo un altro lavoro

d. Don Circostanza riuscì a sbarazzarsi di Berardo perché

(I) gli suggerì di acquistare un buon pezzo di terra

(II) diede a Berardo un piccolo campo sopra Fontamara

(III) gli offrì delle capre invece di un campicello

(IV) in qualche maniera accontentò Berardo

e. Per lavorare quella terra incolta, Berardo dovette

(I) lavorarci dalla mattina alla sera

(II) andarci anche nei giorni di festa

(III) lavorarci durante la notte

(IV) abbandonare il lavoro che faceva di solito con gli altri cafoni

f. I cafoni si rallegrarono con Berardo e bevvero alla sua salute siccome Berardo

(I) era riuscito a fare cose che nessun altro aveva fatto prima di lui 
(II) era salito in cima alla vecchia montagna

(III) aveva trasformato il terreno selvatico in un campo coltivato

(IV) quando si ubriacava, si comportava in un modo leale e sincero.

2. Combina le espressioni con i relativi significati.

a. dare del filo da torcere essere in grave pericolo

b. essere legati a filo doppio trovarsi in una situazione che richiede la massima cautela

c. avere la vita attaccata a un filo essere molto deboli

d. fare il filo a qualcuno/a perdere l'ordine logico

e. tirare le fila di qualcosa mettere in difficoltà

f. reggere le fila di qualcosa dire in ogni particolare

g. essere sul filo del rasoio essere associati in un comune destino

h. raccontare per filo e per segno governare o gestire una cosa

i. stare in piedi coi fili riuscire a risolvere

1. perdere il filo del discorso fare la corte

3. Cambia dal passato remoto al passato prossimo $i$ verbi dei passi seguenti tratti dal testo.

a.r. 1 - 10: Un giorno ... egli balbettò.

b. r. 24 - 29 : Così don Circostanza ... gli altri cafoni.

4. Trasforma, sostituendo il discorso diretto con il discorso indiretto.

a. r. 5 - 7 "Signor avvocato ... per andarci?" 

b. r. 18 - 23 "Ce n'è un'altra ... dal comune."
c. r. 34 - 39 "Tu ti ammazzi ...con le parole."

5. Riempi gli spazi vuoti di questo brano, tratto dallo stesso romanzo, con le forme giuste dei verbi tra parentesi.
Alle quattro del mattino, Berardo e io lasciammo
(lasciare)
Fontamara e verso Fossa per prendere (avviarsi)
il treno di Roma.
Berardo di umore nero e non (essere) neanche al mio buongiorno. $\mathrm{Ma}$ io (rispondere) di non avvedermene per non (fingere)

cominciare a questionare già al momento della partenza. “. la campana stanotte ?" gli (sentire) per attaccare discorso. Però (domandare) come se (essere, parlare)

al vento.

Verso la cappella della Madonna dell'alluvione

“.................. la campana ?" gli ....

Ma neppure mi

Egli svelto e con passi enormi e io sforzarmi per stargli alla pari.

All'entrata di Fossa sorpresi (fare) (sentire, chiedere). (rispondere) (camminare) (dovere) (essere) improvvisamente dal fischio del treno e... (mettersi) a correre per arrivare in tempo a prenderlo. Però 
un treno merci ; al nostro treno

ancora molto.

da una mezz'ora nella sala d'aspetto

della stazione, quando sulla porta

pallido e sconvolto. (essere)

(mancare)

(essere)

(comparire)

(essere)

6. Riempi gli spazi vuoti di questo brano, tratto dallo stesso romanzo, con una delle tre proposte sottoelencate.

Il primo di giugno dell'anno

(1) Fontamara rimase per la prima volta senza illuminazione elettrica. Il due di giugno, il tre di giugno, il quattro di giugno, Fontamara (2) a rimanere senza illuminazione elettrica. (3) nei giorni (4) $\mathrm{e}$ nei mesi seguenti, finché Fontamara si riabituò al regime del (5) di luna. Per arrivare (6) chiaro di luna alla luce elettrica, Fontamara (7) un centinaio di anni, attraverso l'olio di oliva e il petrolio. Per tornare dalla luce elettrica al chiaro di luna (8) una sera.

[...] La luce elettrica era diventata a Fontamara anch'essa una (9) naturale, (10) il chiaro di luna. Nel (11) che nessuno la (12).

1 a) venturo

b) prossimo

c) scorso

2 a) continuò

b) cominciò

c) iniziò

3 a) quando

b) allora

c) $\operatorname{così~}$

4 a) scorsi

b) seguenti

c) prossimi

5 a) riflesso

b) lume

c) chiaro 

6 a) nel
b) dal
c) del
7 a) aveva messo
b) aveva passato
c) aveva preso
8 a) bastò
b) mancò
c) durò
9 a) notizia
b) cosa
c) sorpresa
10a) senza
b) così
c) come
11a) caso
b) paese
c) senso
12a) voleva
b) pagava
c) usava

7. Indica le righe delle sequenze sottospecificate, mettendole secondo l'ordine in cui vengono presentate nel brano.

a. Il duro lavoro di Berardo

b. La disgrazia capitata a Berardo

c. Il rapporto tra Berardo e gli altri suoi compagni

d. Il confronto tra Berardo e l'avvocato

e. I consigli degli altri cafoni e la reazione di Berardo

f. L'avvocato scovato da Berardo

g. La soluzione offerta dall'avvocato.

8. Spunti per la produzione orale e scritta.

a. Secondo te, esiste veramente il destino da cui l'uomo non può scappare ?

b. Scrivi una lettera ad un avvocato per chiedergli un parere legale, spiegando la situazione difficile in cui ti trovi.

c. Ai giorni nostri molti giovani stanno riscoprendo la vita dei campi. Perché, secondo te, c'è questo ritorno alla natura?

d. Quali sono i vantaggi e gli svantaggi della vita del contadino ? 
e. La riuscita dei nostri progetti dipende in larga misura dal nostro carattere e dalla nostra forza d'animo. Commenta.

f. Leggi tutto il romanzo. Riassumi e commenta.

\section{OLTRE IL BRANO}

\section{I segreti dell'efficacia del discorso fra virgolette}

Il giusto dosaggio del discorso diretto nella pagina suppone una tecnica. Una tecnica che è poi una malizia : una malizia che in fin dei conti è un gusto, un certo gusto. Sia come sia, o tecnica o malizia o gusto, la resa in efficacia, quando il dosaggio è "dosato", è indiscutibile : vivacità, agilità.

\section{$[\ldots]$}

Diciamo innanzi tutto che l'uso del discorso diretto, che è poi l'abitudine di dare la parola direttamente al personaggio di cui ci si occupa, è molto comune in quelle inchieste di costume e di caratteri che generalmente sono commissionate ai redattori della terza pagina dei grandi giornali o riviste e che altrettanto generalmente finiscono poi per essere raccolte, riordinate e pubblicate in volume. Grandi produttori di questo genere di articoli sono stati, come noto, Indro Montanelli, Alfredo Pieroni, Giovanni Russo ( per il Corriere della Sera) ; Oriana Fallaci ( per L'Europeo) e Camilla Cederna ( per L'Espresso). Qui, l'abitudine di trasferire direttamente sulla bocca del personaggio intervistato un certo numero di parole è oltretutto un diversivo. 
Che si tratti di un "incontro" alla Montanelli, o di uno "scontro" alla Cederna; che si tratti di un ritratto della grande diva o di un'inchiesta sulla pianificazione in Europa, è sempre bene alternare il personaggio raccontato al personaggio che racconta sé stesso. Tutto ciò dà vita, varietà e interesse alla pagina. Il personaggio viene avanti, va indietro; poi avanti ancora; si sposta di fianco, si siede, si alza, lo si rimette seduto. Questo gioco di prospettive è possibile soltanto al dialogo raccontato che si alterna con il dialogo detto, o meglio riferito.

Il discorso diretto, e cioè il dare la parola direttamente all'intervistato, offre anche questo vantaggio : quello di far dire certe cose troppo forti nel modo meno sgradevole, facendole dire direttamente dall'altro. D'altra parte, parafrasare l'opinione politica di una terza persona significa sempre alterarla in un modo o nell'altro.

\section{[...]}

Molteplici sono gli usi del discorso diretto, e molteplici sono gli effetti che se ne possono ricavare. Se non il più efficace, uno dei più efficaci è quello di usarlo a mo' di chiave di un intero discorso. In questo caso il discorso diretto, ristretto generalmente in forma di sentenza, o di battuta, viene posto in fondo, come un proiettore che illumina tutta la scena del periodo, e illuminandola conclude e spiega in forma lapidaria. E non spiega soltanto ciò che si è tentato di dire, ma anche ciò che si dirà poi. [...]

Poche, secche parole riferite fra virgolette, messe in fondo a un ragionamento, a un episodio hanno il potere di sciogliere, chiarire, di riassumere l'essenza, i termini del discorso stesso.

(da A. FRESCAROLI, Saper scrivere bene oggi, Milano, De Vecchi Editore, 1996, pp. 178-80) 
Opere di IGNAZIO SILONE (Pescina dei Marsi, L'Aquila, 1900 - Ginevra 1978)

Fontamara, 1930 ; Pane e vino, 1936 ; La scuola dei dittatori, 1938 ; Il seme sotto la neve, 1942 ; Una manciata di more, 1952 ; Il segreto di Luca, 1956 ; La volpe e le camelie, 1960 ; Uscita di sicurezza, 1965 ; L'avventura di un povero cristiano, 1968. 


\section{7 \\ PRUDENZA DELLA GUIDA}

Qui dove siamo giunti, l'occhio

può già abbastanza spaziare.

Posiamo i sacchi. Forzare

la marcia ${ }^{1}$, ed avanzare

ancora, più che di saggezza

penso che potrebb'essere un segno,

per tutti noi, di stoltezza ${ }^{2}$.

Si sa che a una certa altezza

10

- è fatale - il ginocchio

è facile alla debolezza.

Non abusiamo ${ }^{3}$. Il prato,

qui sul pianoro ${ }^{4}$, ̀̀ ospitale

più che altrove: ideale

15

anzi (così a mezza quota ${ }^{5}$ :

prima che la mente sia vuota

del tutto) per riprender fiato.

${ }^{1}$ forzare la marcia : spingere oltre la misura normale il modo di camminare con passo ritmicamente accentuato.

${ }^{2}$ stoltezza : mancanza d'intelligenza.

${ }^{3}$ Non abusiamo : non esageriamo, oltrepassando ogni limite.

${ }_{5}^{4}$ pianoro : breve ripiano, quasi orizzontale, sul fianco o sulla cima di un monte.

5 quota : altitudine della montagna. 
Abbiamo camminato,

.

siamone lieti, quel tanto

da poter ora sedere.

Alziamo perciò il bicchiere,

tranquilli, e brindiamo.

$\mathrm{Ma}$, attenti ! non ne traiamo ${ }^{6}$

vanto $^{7}$. Tutti noi conosciamo

le tappe ${ }^{8}$, una per una.

Ma anche - non lo dimentichiamo -

il debito con la fortuna.

Possiamo di qui già vedere

tutto un versante ${ }^{9}:$ abbiamo

dunque già una certezza.

Sostiamo. Che ne sappiamo, noi tutti, di quel che ci aspetta

di là, passata la cresta ${ }^{10}$ ?

Ci sono mormorii ${ }^{11}$

diversi. Voci. Brusii ${ }^{12}$.

${ }^{6}$ traiamo: $1^{\text {a }}$ persona plurale presente del verbo "trarre".

${ }^{7}$ vanto: merito.

${ }^{8}$ tappe : luoghi dove si fanno delle soste per il riposo o il ristoro.

${ }^{9}$ versante : fianco della montagna.

${ }^{10}$ crestá : cima, la parte più alta dei monti.

${ }^{11}$ mormorii : suoni confusi e leggeri prodotti da persone che parlano a bassa voce, (o rumori lievi e continui di acque e correnti).

${ }^{12}$ brusii : rumori confusi e sommessi prodotti da varie persone che parlano e si muovono, (oppure da animali o cose che si muovono). Brusii sono anche della natura, dei torrenti, del vento ecc. 
Non altro. Prima d'avventurarci ${ }^{13}$,

l'insolita sicurezza.

(da GIORGIO CAPRONI, Congedo del viaggiatore cerimonioso

\& altre prosopopee, in Tutte le poesie, Milano, Garzanti, 1999)

1. Vero o Falso?

\section{F}

a. La guida invita i suoi compagni a fermarsi per

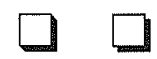
non correre rischi inutili.

b. Continuare sarebbe stata la cosa migliore da fare.

c. Si fermano su un pendio scosceso della montagna.

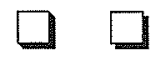

d. Tutti si rallegrano perché la parte più difficile è

e. stata superata.

f. La guida considera se stessa e gli altri escursionisti fortunati, perché aiutati dalla sorte.
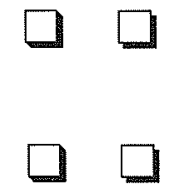

${ }^{13}$ avventurarci : metterci in pericolo. 
f. Pensando alla ripresa del cammino, tutti dovrebbero sentirsi sicuri di se stessi.

g. Secondo la guida, la cosa migliore da fare è andare avanti con i piedi di piombo.

2. Indica con una $\boldsymbol{S}$ i sinonimi e con una $\boldsymbol{C} \boldsymbol{i}$ contrari.
a. giunti (v.1)
arrivati
b. avanzare (v.4)
retrocedere
c. saggezza (v.5)
stupidaggine
d. debolezza (v.11)
stanchezza
e. ospitale (v.13)
accogliente
f. lieti (v.20)
contenti
g. alziamo (v.22)
solleviamo
h. tranquilli (v.23)
preoccupati
i. vanto(v.25)
biasimo
l. conosciamo (v.25)
ignoriamo
m. dimentichiamo (v.27)
ricordiamo
n. certezza (v.32)
sicurezza
o. sostiamo (v.33)
avanziamo
p. passata (v.35)
superata
q. diversi (v.38)
simili
r. insolita (v.41)
rara 
3. Scegli dalle tre alternative quella giusta per spiegare il significato non letterale, ma metaforico dei versi seguenti.

a. qui dove siamo giunti (v.1)

(I) il punto della montagna dove siamo arrivati

(II) il momento presente della nostra vita

(III) il luogo dove la guida ci dice di fare una sosta

b. Posiamo i sacchi (v.3)

(I) ci fermiamo per fare delle riflessioni

(II) ci fermiamo per riprendere fiato

(III) ci fermiamo perché i sacchi sono troppo pesanti

c. a una certa altezza - è fatale - (vv.9-10)

(I) è pericoloso andare oltre i limiti umani

(II) salire ancora può causare vertigini

(III) andare più in alto è rischioso

d. il prato, qui sul pianoro (vv.12-13)

(I) una zona pianeggiante

(II) un luogo di divertimento

(III) un luogo dove ci si sente al sicuro

e. non ne traiamo vanto (vv.24-25)

(I) non abbiamo motivo per sentirci fieri

(II) dobbiamo sempre essere cauti e prudenti

(III) le persone che ci conoscono, ci lodano 
f. tutti noi conosciamo le tappe, una per una (vv.25-26)

(I) ci ricordiamo delle soste che abbiamo fatto

(II) teniamo un diario dettagliato

(III) siamo consci di tutti gli eventi importanti della nostra vita

g. il debito con la fortuna (v.28)

(I) la riconoscenza verso la nostra buona sorte

(II) la fortuna ha un debito con noi

(III) il destino ce l'ha fatta pagare

h. quel che ci aspetta di là, passata la cresta ? (vv.34-35)

(I) i problemi che sorgeranno quando continueremo l'escursione

(II) la paura che in futuro la gente ci potrà trattare male

(III) la vita futura è un'incognita

4. Accoppia le espressioni della prima colonna con il rispettivo significato dato nella seconda.

a. prendere per moglie

b. prendere qualcuno per ladro

c. prendere il ladro

d. prendere un granchio

e. prendersela con qualcuno sbagliare in modo grossolano

trattare qualcuno nel modo e con il tono più adatto

sfogare su qualcuno la propria rabbia e il proprio risentimento provare un'improvvisa e violenta passione amorosa

sorprendere qualcuno mentre fa qual cosa di male 
f. prendere piede

g. prendere per il verso giusto

h. prendere qualcuno con le mani nel sacco

i. prendere qualcuno in casa

1. prendere una cotta

m. prendere con le molle scegliere qualcuno come ospite

scambiare qualcuno per

trattare con estrema cautela una cosa oppure una persona difficile o poco raccomandabile

catturare diffondersi, rafforzarsi sposare

5. Abbina i termini della prima colonna con il loro significato dato nella seconda.

a. scricchiolìo

b. gocciolìo

c. ronzìo

d. calpestìo

e. formicolìo

f. fruscìo il rumore continuo e monotono prodotto dal passaggio di molte persone o animali il rumore indistinto di parole dette in modo sommesso e confuso

la sensazione della pelle simile al contatto di formiche, associata alla perdita di sensibilità e talvolta all'impressione di bruciore il rumore prodotto dal trascinare qualcosa in maniera continuata e poco energica

il continuo cadere di piccolissime quantità di un liquido

il muoversi confuso e apparentemente senza scopo che fanno grandi quantità di insetti, animali, o persone 
g. strascichìo

h. sussurrìo

i. borbottìo

I. brulichìo

m. sfarfallìo il rumore monotono e vibrante emesso nel volare da mosconi, api, vespe e calabroni la fastidiosa mancanza di continuità delle immagini sullo schermo o lo svolazzo continuo di farfalle o di altri insetti provvisti di ali

il suono secco e crepitante di cosa dura che si fende e si rompe

il rumore indistinto e continuo di persone che parlano a bassa voce il rumore sommesso e strisciante, prodotto da tessuti, foglie o carte che si muovono

Attenzione : da notare il passaggio in queste parole da -ìo al singolare ad -ii al plurale, poiché l'accento cade sulla -i- di -ìo.

6. Specifica a chi o a che cosa rimandano i pronomi sottolineati.
a. siamone lieti (v.20)
b. non ne traiamo vanto (vv.24-25)
c. non lo dimentichiamo (v.27)
d. che ne sappiamo (v.33)
e. di quel che ci aspetta (v.34) 
7. Nel brano seguente, tratto da uno studio di Giovanni Raboni, sono stati inseriti alcuni errori di natura morfologica. Correggili.

[...] I tre temi (della città, delle madre, del viaggio) appaiono, nella vita reala dei singoli testi e del testo complessivo che lo risulta, così intrecciati, così ribaditi l'uno all'altro, e l'uno nel altro da formare, più che una successione, un'anello di temi - o, se si vuole, una sistema di temi leggibili anche come sinonimi, o meglio come anagrammi, l'uno dell'altro, un po' come nei vertiginosi giochi di simmetrie e di specchi usate nelle loro partiture dai polifonisti della scuola fiamminga. $\grave{E}$ certo, comunque, che $i$ tre temi hanno un comune denominatore, che è quel dell'esilio. Esilio dal spazio (la città), dal tempo passata ( la madre), dalla vita (il viaggio). Esilio dal quale, e del quale, il poeta ci parla per mezzo della "rondine" delle suoi ballate, delle sue rime. E che fa dell'intera sua opera poetica (se vogliamo ricorrere per una formula) un grande, struggente e severo canzoniere d'esilio $o$, in altro senso, un'ininterrotto diario di viaggio: viaggio nel tempo e allo spazio, viaggio nel nulla (nella nebbia, nell'Ade) ricordando la madre e la terra, viaggio nel tunnel dell'assenza di Dio assaporando l'amaro trionfo della sua scomparsa, viaggio nell'antimateria - nel non-spazio, non-tempo, non-luogo - capovolgendo (e al tempo stesso celebrando con raggelata e affettuosa ironia) l'appuntamenti, gli riti, le "cerimonie" dell'ovvietà quotidiani. 
8. Riscrivi in prosa questa poesia di Giorgio Caproni e spiegane il significato.

\section{LO SPATRIATO*}

Lo hanno portato via

dal luogo della sua lingua.

Lo hanno scaricato male

in terra straniera.

Ora, non sa più dove sia

la sua tribù. È perduto.

Chiede. Brancola. Urla.

Peggio che se fosse muto.

9. Spunti per la produzione orale e scritta.

a. Una gita in montagna

b. Racconta un'esperienza indimenticabile che termina con queste parole : "... e da quel giorno non ci sono più ritornato".

c. Dopo aver letto la poesia L'infinito di Giacomo Leopardi, parla di quello che, secondo te, c'è oltre "la siepe".

d. Italo Calvino, in un articolo su Caproni apparso sul giornale la Repubblica scrive : "L'allegoria dell'addio al mondo s'innesta su di una semplicissima situazione ferroviaria : un passeggero che saluta i compagni di scompartimento prima di scendere alla sua stazione". Commenta.

* Una persona mandata o andata via dalla patria, dal Paese natale. 
e. Scrivi una breve biografia di Giorgio Caproni e riporta tutti i dati seguenti :

- Luogo e anno di nascita : Livorno, 1912

- Infanzia e gioventù : a Genova

- Studi : quelli musicali, interrotti ; si dedica presto alla letteratura

- 1939 : richiamato alle armi ; con i partigiani fino alla Liberazione

- A Roma : critico della Fiera Letteraria, del Punto, e della Nazione, oltre che traduttore

- 1982 : Premio Feltrinelli per la poesia consegnato dall'Accademia dei Lincei.

- Alcune opere : Il seme del piangere (1959), Il muro della terra (1975), Il labirinto (1984)

- Luogo e data di morte: Roma, 22 gennaio 1990.

\section{OLTRE IL BRANO}

\section{Metafore}

Uno stupendo strumento del linguaggio è l'uso espressivo della metafora che, sulla base di un'intuizione di similarità tra due elementi, rende possibile costruire e comunicare immagini e presenze anche fortemente soggettive. La formazione di metafore trova un terreno fertile nella visione animistica e antropomorfica del mondo e si rivela uno dei mezzi più efficaci 
per trasmettere un'emozione in quanto più direttamente capace di coinvolgere la partecipazione e la sensibilità dell'ascoltatore/lettore. [...]

La metafora fa violenza alla realtà e ne produce una nuova attraverso similitudini che non hanno alcuna evidenza immediata e giungono a costruire nuovi rapporti tra le cose. Ciò facendo, la metafora, naturalmente, deve presupporre che l'ascoltatore sia consapevole dell'universo di finzione che è stato instaurato. [...]

La potenzialità creativa della metafora è talmente forte da postulare nuove realtà : se cielo e terra sembrano attaccati come una casa a due piani, il cielo è un tetto azzurro ${ }^{1}$; se Zhen Niang aspetta il suo amato salda come una roccia, si trasforma in una statua dal viso proteso in avanti che sembra dire "Han tu, dove sei ?"2.

Anche la parola cessa di rinviare a ciò che designa e diventa essa stessa realtà, le parti del linguaggio sono tramutate in oggetti. Perrault descrive le metamorfosi delle parole uscite dalla bocca della fanciulla buona e di quella cattiva dopo l'incontro con la fata : ad ogni frase, due rose, due perle e due grossi diamanti escono dalle labbra della più piccola, due vipere, due rospi da quella maggiore ${ }^{3}$. [...]

Un altro elemento molto interessante è la "messa in scena" di espressioni linguistiche. "Ho male alla testa" è drammatizzato da un fantasma che batte con un martello un ferro appuntito piantato sulla testa. Il fedele Enrico è così afflitto che si fa mettere tre cerchi intorno al cuore perché

\footnotetext{
1 "Come sono venute le stelle", in Fiabe africane, a cura di P. Radin, Torino, Einaudi, 1984, p.42.

2 "Wang Fu Shi", in Antiche fiabe cinesi, a cura di E. Bozza, Milano, Mondadori, 1988, p. 184.

3 "La fata", in Ch. Perrault, I racconti di Mamma l'Oca, Torino, Einaudi, 1980, pp. 25-26.

4 "Una prova inconfutabile", in Fiabe e storie cinesi, a cura di M. Roberts, Milano, Arcana, 1986, p.214.
} 
non gli scoppi". "Bussare alla porta del cuore" determina la trasformazione di questo organo in uno spazio fisico abitato e frequentato ${ }^{6}$.

Il "teatro" dei modi di dire a volte sceneggia puntualmente le espressioni. Per esempio due angeli portano via la trave che uno s'era tenuto nell'occhio mentre cercava la pagliuzza in quello degli altri ${ }^{7}$. A volte le interpreta in modo ironico come "essere senza testa" nel senso di essere distratti $[\ldots]$

A parte il potere magico delle parole che modifica e domina la realtà, esiste comunque una forza misteriosa nel linguaggio. Grazie a questa forza un proclama emesso dall'imperatore, dove si dichiara che la distanza dal villaggio alla città deve considerarsi non più di dieci miglia ma soltanto di cinque, è accolto con grande entusiasmo dai sudditi ai quali da quel giorno la strada appare più breve e meno faticosa ${ }^{8}$.

Il gioco con le parole e i significati di queste offrono moltissime possibilità. Come fa Ulisse con Polifemo, la figlia furba del taglialegna induce un mercante a credere che il suo nome è "Nessuno-in nessun postoniente" ; e quando lui urla a squarcia gola che è stato derubato, alle domande della gente "Chi ti ha derubato ? Dove sei stato derubato ? Che cosa ti hanno rubato?", non può che rispondere: "Nessuno-in nessun postoniente" $"$.

(da M.R. ALESSANDRI, Manuale del fantastico, Firenze, La Nuova Italia, 1992, pp.17-21)

\footnotetext{
5 "Il principe ranocchio o Enrico di ferro", in J. e W. Grimm, Fiabe, vol. I, Milano, Mondadori, 1984, p.5.

6 "Il tumulo", ivi, vol. III, p.186.

7 "Mastro Punteruolo", ivi, p.126.

8 "Cinque avventure di uno sciocco", in Enciclopedia della favola, vol. III, a cura di G. Rodari, Roma, Editori Riuniti, 1970, p.173.

9 "Il taglialegna e la figlia furba", ivi, p.22.
} 


\section{OPERE DI GIORGIO CAPRONI ( Livorno 1912 - Roma 1990)}

Come un'allegoria, 1936 ; Ballo a Fontanigorda, 1938 ; Cronistoria, 1943 ;

Stanze della funicolare, 1952; Il passaggio di Enea, 1956; Il seme del piangere, 1959 ; Congedo del viaggiatore cerimonioso \& altre prosopopee, 1965 ; Il muro della terra, 1975 ; L'ultimo borgo, 1980 ; Il franco cacciatore, 1982; Il labirinto, 1984; Il conte di Kevenhïller, 1986; Res amissa, postuma, 1991. 


\section{8 \\ UN PO' DI NEBBIA}

Del primo inverno, se rigido ${ }^{1}$, piovoso, nebbioso, quasi non m'ero accorto tra gli svaghi ${ }^{2}$ de ${ }^{3}$ viaggi e nell'ebbrezza ${ }^{4}$ della nuova libertà. Ora questo secondo mi sorprendeva già un po' stanco, come ho detto, del vagabondaggio e deliberato ${ }^{5}$ a impormi un freno ${ }^{6}$. E mi accorgevo che ... sì, 5 c'era un po' di nebbia, c'era; e faceva freddo ; m'accorgevo che per quanto il mio animo si opponesse a prender qualità dal colore del tempo, $\operatorname{pur}^{7}$ ne soffriva.

"Ma sta' a vedere," mi rampognavo ${ }^{8}$ " "che non debba più far nuvo$10^{9}$ perché tu possa ora godere serenamente della tua libertà !"

M'ero spassato $^{10}$ abbastanza, correndo di qua e di là : Adriano Meis ${ }^{11}$ aveva avuto in quell'anno la sua giovinezza spensierata ; ora bisognava che diventasse uomo, si raccogliesse in sé, si formasse un abito di

\footnotetext{
${ }^{1}$ rigido: molto freddo.

${ }^{2}$ svaghi : divertimenti.

${ }^{3} \mathrm{de}^{\prime}$ : dei.

${ }^{4}$ ebbrezza: stato di grande entusiasmo.

${ }^{5}$ deliberato : deciso.

${ }_{7}^{6}$ freno: limite.

${ }^{7}$ pur : tuttavia

${ }^{8}$ mi rampognavo: mi rimproveravo.

${ }^{9}$ far nuvolo : annuvolarsi.

${ }^{10}$ M'ero spassato : mi ero divertito.

${ }^{11}$ Adriano Meis : il protagonista, Mattia Pascal, quando legge sul giornale la notizia del proprio suicidio (in quanto è stato identificato nel cadavere di un annegato, al suo paese), approfitta della coincidenza per eliminare il suo passato e cominciare una nuova vita col nome di Adriano Meis.
} 
vita quieto e modesto. Oh, gli sarebbe stato facile, libero com'era e senz'obblighi di sorta ${ }^{12}$ !

Così mi pareva; e mi misi a pensare in quale città mi sarebbe convenuto di fissar dimora ${ }^{13}$, giacché ${ }^{14}$ come un uccello senza nido non potevo più oltre rimanere, se proprio dovevo compormi ${ }^{15}$ una regolare esistenza. Ma dove ? in una grande città o in una piccola ? Non sapevo risolvermi ${ }^{16}$.

Chiudevo gli occhi e col pensiero volavo a quelle città che avevo 20 già visitate; dall'una all'altra, indugiandomi ${ }^{17}$ in ciascuna fino a rivedere con precisione quella tal via, quella tal piazza, quel tal luogo, insomma, di cui serbavo ${ }^{18}$ più viva memoria; e dicevo :

"Ecco, io $\mathrm{vi}^{19}$ sono stato! Ora, quanta vita mi sfugge, che séguita ad agitarsi qua e là variamente. Eppure, in quanti luoghi ho detto :- Qua vor-

25 rei aver casa ! Come ci vivrei volentieri ! - E ho invidiato gli abitanti che, quietamente, con le loro abitudini e le loro consuete ${ }^{21}$ occupazioni, potevano dimorarvi ${ }^{22}$, senza conoscere quel senso penoso di precarietà ${ }^{23}$ che tien sospeso $^{24}$ l'animo di chi viaggia."

${ }^{12}$ di sorta : di nessun tipo.

${ }_{14}^{13}$ fissare dimora : stabilirmi, abitare.

${ }^{14}$ giacché : dal momento che, poiché.

${ }^{15}$ compormi : costruirmi.

${ }^{16}$ risolvermi : decidermi.

${ }^{17}$ indugiandomi : fermandomi più del solito.

${ }^{18}$ serbavo : conservavo.

${ }^{19} v i$ : ci.

${ }^{20}$ séguita : continua.

${ }^{21}$ consuete : solite.

22 dimorarvi : viverci.

${ }_{23}^{23}$ precarietà : instabilità.

${ }^{24}$ tien sospeso : lascia in uno stato di incertezza tormentosa. 
Questo senso penoso di precarietà mi teneva ancora e non mi faceva 30 amare il letto su cui mi ponevo a dormire, $\mathrm{i}$ varii ${ }^{25}$ oggetti che mi stavano intorno.

Ogni oggetto in noi suol ${ }^{26}$ trasformarsi secondo le immagini ch'esso evoca $^{27}$ e aggruppa ${ }^{28}$, per così dire, attorno a sé. Certo un oggetto può piacere anche per se stesso, per la diversità delle sensazioni gradevoli che ci su35 scita in una percezione armoniosa; ma ben più spesso il piacere che un oggetto ci procura non si trova nell'oggetto per se medesimo. La fantasia lo abbellisce cingendolo ${ }^{29}$ e quasi irraggiandolo ${ }^{30}$ d'immagini care. Né noi lo percepiamo $^{31}$ più qual esso è, ma così, quasi animato dalle immagini che suscita in noi o che le nostre abitudini vi associano. Nell'oggetto, insomma,

40 noi amiamo quel che vi mettiamo di noi, l'accordo, l'armonia che stabiliamo tra esso e noi, l'anima che esso acquista per noi soltanto e che è formato dai nostri ricordi.

Or come poteva avvenire per me tutto questo in una camera d'albergo ?

Ma una casa, una casa mia, tutta mia, avrei potuto più averla ? I miei denari erano pochini ... Ma una casettina modesta, di poche stanze ? Piano : bisognava vedere, considerar bene prima, tante cose. Certo, libero, liberissimo, io potevo essere soltanto così, con la valigia in mano : oggi qua, domani là. Fermo in un luogo, proprietario d'una casa, eh, allora : registri e

\footnotetext{
${ }^{25}$ varii : vari.

${ }^{26} \mathrm{suol}$ : terza persona indicativo presente del verbo "solere", è solito.

${ }^{27}$ evoca: richiama alla mente.

${ }_{28}^{28}$ aggruppa: raggruppa, riunisce in uno o più gruppi.

${ }^{29}$ cingendolo : attorniandolo.

${ }^{30}$ irraggiandolo : illuminandolo.

${ }^{31}$ lo percepiamo: ne acquistiamo coscienza per mezzo dei sensi o dell'intuito.
} 
50 tasse subito ! E non mi avrebbero iscritto all'anagrafe ${ }^{32}$ ? Ma sicuramente ! E come? con un nome falso ? E allora, chi sa ?, forse indagini segrete intorno a me da parte della polizia ... Insomma, impicci ${ }^{33}$, imbrogli $^{34} ! \ldots$ No, via : prevedevo di non poter più avere una casa mia, oggetti miei. Ma mi sarei allogato $^{35}$ a pensione in qualche famiglia, in una camera mobiliata. 55 Dovevo affliggermi ${ }^{36}$ per così poco ?

L'inverno, l'inverno $\mathrm{m}$ 'ispirava queste riflessioni malinconiche, la prossima festa di Natale che fa desiderare il tepore ${ }^{37}$ d'un cantuccio ${ }^{38}$ caro, il raccoglimento, l'intimità della casa.

(da LUGI PIRANDELLO, Il fu Mattia Pascal, a c. di

Claudio Toscani, Milano, Mondadori, 1990)

32 anagrafe : registro comunale ove sono trascritti, riportati e conservati i dati relativi a nascita, residenza, stato civile, matrimonio, morte, ecc. di ciascun cittadino. $\grave{E}$ anche l'ufficio dove si conserva tale registro e dove si rilasciano i relativi certificati. 33 impicci : fastidi, guai.

${ }^{34}$ imbrogli : inganni.

${ }^{35}$ allogato : alloggiato.

${ }^{36}$ affliggermi : addolorarmi.

${ }^{37}$ tepore : calore gradevole.

38 cantuccio : angolo riposto di una stanza. 
1. Dalle quattro alternative, scegli le due giuste e mettile in ordine a seconda della loro importanza.

a. Il protagonista considerava il secondo inverno peggiore del primo perché :

(I) era stanco di andare di città in città

(II) pativa il freddo più dell'inverno precedente

(III) gli dava fastidio la nebbia

(IV) soffriva di solitudine

b. Adriano Meis voleva stabilirsi in una città perché :

(I) girando, aveva trovato quella ideale

(II) aveva voglia di una vita più ordinata

(III) la vita spensierata dell'anno precedente non lo attirava più

(IV) sentiva che stava invecchiando

c. Ripensando alle città visitate, Meis ricordava soprattutto :

(I) le varie vie e le piazze

(II) una casa che l'aveva colpito e che avrebbe voluto per sé

(III) gli abitanti dei quali invidiava la vita ordinata

(IV) la stanchezza che sentiva quando era in viaggio

d. Le cose che circondano l'uomo, acquistano importanza secondo :

(I) l'impronta personale che dà l'uomo a queste cose

(II) il modo in cui vengono acquistate

(III) la loro bellezza

(IV) i ricordi che suscitano

e. Per Adriano Meis, l'esigenza di avere una casa nasceva :

(I) dal freddo invernale che lo faceva star male 
(II) dall'atmosfera natalizia che lo circondava e lo faceva sentire solo

(III) dall'amara realtà che gli erano rimasti pochi soldi

(IV) dalla voglia di dar un senso alla propria vita

2. Individua nel testo, in ordine progressivo, le parole che hanno $i$ seguenti significati :

da r.1 a r.18 :

a. stato di stordimento provocato da sensazioni molto intense

b. vita di chi non ha un'abitazione stabile e passa da un posto all'altro in continuazione

da r.19 a r.31 :

c. soffermandomi

d. incertezza

da r.32 a r. 44 :

e. richiama alla mente

f. circondandolo

da r.45 a r.58 :

g. registro o archivio contenente i dati sulla popolazione di ogni comune italiano

h. addolorarmi, tormentarmi.

3. Scrivi due frasi in cui è presente ciascuna parola, ma con un significato differente.

nuova (r.2) - aggettivo / nome

secondo (r.3) - aggettivo / nome 


$$
\begin{aligned}
& \text { tempo (r.6) - nome / nome } \\
& \text { abito (r.12) - nome / verbo } \\
& \text { vita (r.13) - nome / nome } \\
& \text { senso (r.27) - nome / nome } \\
& \text { piacere (r.35) - nome / verbo } \\
& \text { care (r.37) - aggettivo / aggettivo } \\
& \text { ricordi (r.42) - nome / verbo } \\
& \text { piano (r.47) - avverbio / nome } \\
& \text { parte (r.52) - nome / verbo } \\
& \text { imbrogli (r.52) - nome / verbo } \\
& \text { pensione (r.54) - nome / nome }
\end{aligned}
$$

4. Forma dei nomi dai seguenti aggettivi e inseriscili in frasi di senso compiuto.

rigido (r.1), piovoso (r.1), nebbioso (r.1), nuova (r.2), stanco (r.3), spensierata (r.11), quieto (r.13), modesto (r.13), libero (r.13), penoso (r.27), armoniosa (r.35), falso (r.51), malinconiche (r.56).

5. Trasforma il primo paragrafo (r.1-7) e il quarto paragrafo (r.1518) dalla prima persona alla terza persona singolare.

6. Rintraccia nel testo tutti $i$ verbi al congiuntivo e al condizionale, specificandone i tempi. 
7. Dal seguente brano critico di Claudio Toscani, tratto dalla stessa edizione del romanzo sopraindicata, sono stati omessi i sostantivi sottoelencati. Inseriscili negli spazi vuoti.

La prima esigenza è quella di un ................ físso di una casa se non di una ................ Ma dove ? Soltanto lui, da che ha iniziato a vivere sulla $\ldots \ldots \ldots \ldots \ldots \ldots$ di un morto, è estraneo in ogni e in ogni $\ldots . . . \ldots \ldots \ldots \ldots$; solamente lui è "forestiere della vita". [ ...]

I ............... gli son venuti a ............... e la "penosa precarietà" di chi gira continuamente per il ................ fa in Adriano Meis tutt'uno con la precarietà categoriale del suo essere, o sarebbe più giusto dire, non essere. Il fastidio per gli ............... diventa pian piano fastidio per la stessa .................

famiglia, luogo, momento, mondo, noia, pelle, punto, spostamenti, viaggi, vita.

8. Riscrivi le frasi seguenti: (a) sostituendo $i$ verbi sottolineati con nomi che abbiano la stessa radice; (b) apportando $i$ necessari cambiamenti ; (c) mantenendo il significato di partenza (es. E non mi avrebbero iscritto all'anagrafe ? (r.50): E non mi avrebbero fatto l'iscrizione all'anagrafe ?).

a) Ora bisognava che diventasse uomo (r.11-12) 
b) Ho invidiato gli abitanti che, quietamente, con le loro abitudini e le loro consuete occupazioni, potevano dimorarvi (r.25-27)

c) Ogni oggetto in noi suol trasformarsi secondo le immagini ch'esso evoca (r.32-33)

d) Nell'oggetto, insomma, noi amiamo quel che vi mettiamo di noi (r.39-40)

e) Prevedevo di non poter più avere una casa mia, oggetti miei (r.53)

f) L'inverno m'ispirava queste riflessioni malinconiche (r. 56)

9. Adriano Meis ha girato per tante città. Immagina e racconta uno di questi suoi viaggi, mettendo in rilievo le sensazioni che prova questo personaggio mentre gira tutto solo.

10. Spunti per la produzione orale e scritta.

a. Racconta gli aspetti più interessanti di un viaggio che hai fatto.

b. Racconta una fiaba natalizia del tuo Paese.

c. Secondo te, fino a che punto l'uomo può veramente considerarsi un essere libero ?

d. L'incontro, dopo tanto tempo, con un/a amico/a.

e. Scrivi una lettera ad un'agenzia immobiliare per informare il responsabile che stai cercando casa, descrivendogli dove e che tipo di casa vorresti acquistare.

f. Parla di una città che ti è particolarmente cara, descrivendo

- il suo assetto urbanistico

- i monumenti di interesse storico e/o artistico 
- i posti da visitare

- il modo di vivere della gente

- le specialità gastronomiche.

\section{OLTRE IL BRANO}

\section{L'uomo senza unità}

La coscienza del vivere è [...] la dimensione nuova di Mattia Pascal. La vita è prigione assurda di forme provvisorie e vane, eppure oppressive e alienanti ; la società inchioda l'uomo a una falsa individuazione, che ne snatura i desideri e la volontà, frantuma l'unità della coscienza in una molteplicità menzognera. Egli, allora, si ribella, evade, rifiuta le convenzioni e l'artificio di una maschera odiata. Intraprende il suo viaggio di redenzione, cercando altre individuazioni più vere, la libertà, l'amore autentico, la giustizia, l'onore : e gode finalmente del suo senso di disponibilità illimitata, del suo ritorno ad una coscienza pura e senza condizioni [...].

Ma è breve ebrezza : ché l'amore vero è rapporto, la giustizia è relativo confronto, la coscienza sono gli altri in noi. La più tragica delle delusioni offende quella aspirazione, una definitiva impossibilità di individuazione disillude quella pura volontà di scelta : ogni esperienza riconduce l'uomo alla necessità di un patteggiamento continuo, di una compromissio- 
ne fuori della quale la libertà è arbitrio, la pura disponibilità è reale inconsistenza. Un più incolmabile caos inghiotte l'uomo nella sua evasione dal caos degli uomini. Ed egli non può che tornare sui suoi passi, rivestire uno ad uno i panni dimessi della vecchia mascherata, cercare di ricomporre i frammenti di quella sua antica forma : provvisoria e falsa, ma alla fine l'unica possibile, l'unica nella quale la vita possa tuttavia consistere e misurarsi (“... fuori della legge e fuori di quelle particolarità, liete o tristi che siano, per cui noi siamo così, ... non è possibile vivere". Il fu Mattia Pascal).

Lungi dal pacificare l'avventura del personaggio in un accettato "trionfo dello stato civile", questa conclusione è l'immediata premessa del dramma definitivo dell'uomo di Pirandello. Giacché il fallimento del tentativo anarchico di Mattia Pascal non è solo la provvisoria, sperimentale evasione dell'uomo dalla prigione delle forme convenzionali, dalla società che lo mistifica, alla ricerca di una autenticità umana che lo renda libero e padrone di sé ; ma è anche, e soprattutto, la scoperta della tragica necessità di quella forma opprimente, di quella inumana prigione. Al di là del suo umoristico ritorno, del suo reinserirsi imbronciato nella macchina oscura e alienatrice dei patti sociali, è proprio la sua breve esperienza di svincolato, di "forestiere della vita", che prefigura con sconcertante intuizione il dramma autentico delle grandi creature di Pirandello. La ribellione alle maschere false, in cui sembra risolversi tutta la carica polemica della prima "folla" pirandelliana, conduce quella folla, attraverso Mattia Pascal che la rappresenta tutta, ad una esperienza ben più devastante, ad un rischio di nullificazione totale. Il culmine di questa esperienza coincide col momento psicologico in cui, tutto posseduto dalla trasparente letizia di una libertà assoluta, di una coscienza della vita senza condizioni, l'eroe per vivere, comincia ad avvertire l'esigenza di fabbricarsi un nuovo sentimento della vita, e dunque, di fingere, di inventare una nuova forma [...]. Evadere è dunque impossibi- 
le, vivere è dunque impossibile, se non nei rapporti falsi, nel relativo del caos sociale ; fuori di ciò c'è il nulla [...]. Il ritorno alla vita dell'eroe pirandelliano si colora così di una ben più profonda amarezza e acquista il significato e la consapevolezza di una condanna infame : la scoperta della impossibilità di ogni evasione, che si riduce ad un salto nel buio.

(da A. LEONE DE CASTRIS, Storia di Pirandello, Bari, Laterza, 1978, pp.74-77)

\section{Opere di LUIGI PIRANDELLO (Girgenti, oggi Agrigento, 1867 - Ro- ma 1936)}

Mal giocondo, 1889; L'esclusa, 1901; Il fu Mattia Pascal, 1904; L'umorismo, 1908 ; I vecchi e i giovani, 1913 ; Liolà, 1917 ; Il berretto a sonagli, 1918 ; Così è (se vi pare), 1918 ; La patente, 1918 ; Sei personaggi in cerca d'autore, 1921 ; Enrico IV, 1922 ; Vestire gli ignudi, 1923 ; Ciascuno a suo modo, 1924 ; La giara, 1925 ; Quaderni di Serafino Gubbio operatore, 1925 ; Uno, nessuno e centomila, 1925-26 ; L'uomo dal fiore in bocca, 1926; Questa sera si recita a soggetto, 1930 ; Trovarsi, 1932 ; I giganti della montagna, postumi 1938. 


\section{9 \\ L'AIRONE}

Si mise a scrutare ${ }^{1}$ anche lui il cielo, allora, nella stessa direzione; e vide quasi subito un uccello isolato che, a un centinaio di metri di quota ${ }^{2}$, avanzava lentamente verso di loro.

"Che cos'è ?" domandò.

"Dovrebbe essere un airone", disse Gavino.

Si trattava di un uccello piuttosto grosso : con due ali grandi, molto grandi, però sproporzionate rispetto al corpo, che era piccolo, invece, graci$1 e^{4}$. Stava volando con fatica evidente, arrancando ${ }^{5}$. Il lungo collo a esse ${ }^{6}$, stretto fra le scapole ${ }^{7}$; le vaste ali marrone, di una pesantezza da stoffa, a-

10 perte a tirarsi sotto la pancia il maggior volume di aria possibile : sembrava non farcela a tagliare di traverso il vento, ed anzi in procinto ${ }^{8}$, ad ogni istante, di venire travolto, d'essere spazzato via come uno straccio?.

"Che buffa bestia !", pensò.

\footnotetext{
${ }^{1}$ scrutare: osservare con attenzione.

${ }^{2}$ quota : altezza dal suolo.

3 airone : uccello di notevoli dimensioni, con gambe lunghe e sottili, ali larghe, collo incurvato a $\mathrm{S}$, becco lungo e ciuffo sul capo. Vive sulle rive delle acque interne $\mathrm{e}$ mangia animali acquatici.

${ }_{5}^{4}$ gracile: magro e delicato.

5 arrancando: procedendo con difficoltà.

6 a esse : a forma della lettera S che si pronunzia "esse".

${ }^{7}$ scapole : due ossa piatte, triangolari, applicate alla parete posteriore del torace.

${ }^{8}$ in procinto: sul punto.

${ }^{9}$ straccio : cencio, brandello di stoffa.
} 
Lo vide sorvolare adagio il pezzo di laguna che separava la barena ${ }^{10}$ dalla botte ${ }^{11}$, e quindi sospendersi a perpendicolo sopra le loro teste : fermo, in pratica, e perdendo via via un po' di quota. [...]

Veniva avanti, adesso, sempre più avanti, mostrandoglisi - e il cuore, frattanto, aveva cominciato a battergli forte contro l'osso dello sterno ${ }^{12}$-, con straordinaria, quasi insopportabile evidenza. Sulla testina perfettamente 20 liscia, inalberava ${ }^{13}$ per di dietro qualcosa di esile : una specie di filo, di antenna, chi lo sa. E lui stava appunto chiedendosi che cosa diamine ${ }^{14}$ potesse essere, quello strano affare, e stringeva le palpebre per cercare di veder meglio, quando, improvvisamente, nella vasta aria soleggiata e ventosa, udì echeggiare il solito doppio sparo.

Non cadde subito. Lo vide come sussultare ${ }^{15}$, lassù in alto, sbattere disordinatamente le ampie ali marrone, quindi sbandare ${ }^{16}$ verso l'isolotto da cui erano partite le fucilate. Lottava per sostenersi, per riprender quota. Ma poi si lasciò andare, di colpo, e venne giù come se stesse rompendosi in tanti pezzi. $[\ldots]$

Credeva che fosse morto, e che la cagna si sarebbe avventata ${ }^{17}$ a raccoglierlo. Invece no : appena riemerso, si drizzò immediatamente su quei suoi trampoli di gambe ${ }^{18}$, cominciando a muovere in qua e in là, a scatti, la testina minuscola. "Dov'è che mi trovo ?", aveva l'aria di chiedersi. "E cosa mi è successo ?"

\footnotetext{
${ }^{10}$ barena : tratto del fondo lagunare che emerge quando c'è la marea bassa.

${ }^{11}$ botte : appostamento o riparo a forma di botte superiormente aperta, usato dai cacciatori in palude.

12 sterno : osso piatto del torace al quale si uniscono le coste o costole.

${ }^{13}$ inalberava: alzava in modo che stesse su dritta.

${ }^{14}$ diamine : esclamazione di meraviglia.

${ }^{15}$ sussultare : fare un movimento improvviso dal basso verso l'alto e viceversa.

${ }^{16}$ sbandare: uscire dalla rotta.

17 avventata: lanciata con impeto.

${ }^{18}$ trampoli di gambe : gambe molto lunghe.
} 
Inquieto, senza mai smettere di volgere in giro la liscia testa un po' fatua $^{19}$, da viveur ${ }^{20}$, prolungata dietro la nuca dalla strana, quasi impercettibile $^{21}$ antenna filiforme ${ }^{22}$, cercava tuttavia di raccapezzarsi ${ }^{23}$, di riconoscere, se non i luoghi, almeno la natura degli oggetti che lo circondavano. Lontano pochi passi, per esempio, mezzo all'asciutto e mezzo nell'acqua, aveva notato il vulicipio ${ }^{24}$. Che cos'era ? Una barca, oppure il corpo di un grosso animale addormentato ? Alla larga, comunque. Meglio non azzardarsi a raggiungere la spiaggetta di sabbia fine e compatta dove quel $\operatorname{coso}^{25}$ scuro e minaccioso giaceva di traverso : molto meglio. La fitta ${ }^{26}$ al fianco, del resto, non la avvertiva neanche più. L'ala bastava non muoverla, e non gli faceva male. Poteva aspettare.

Lo guardava pieno di ansia, immedesimandosi ${ }^{27}$ totalmente. Anche a lui sfuggiva il perché di tante cose. Per qual motivo Gavino aveva sparato ? E perché non si alzava in piedi, adesso, e non tirava un colpo supplementare, quello di grazia ? Non era questa la regola ? E la cagna ? Che cosa temeva, Gavino : che l'airone, non ancora dissanguato ${ }^{28}$ a sufficienza, potesse servirsi del becco per difendersi ? E l'airone ? Che cosa avrebbe fatto?

\footnotetext{
19 fatua: vanitosa.

${ }^{20}$ viveur : francesismo, uomo di mondo, uno che si gode la vita.

${ }^{21}$ impercettibile: che non si nota oppure si nota appena.

${ }^{22}$ filiforme : che ha forma di filo, in quanto allungata e sottile come un filo.

${ }^{23}$ raccapezzarsi : arrivare a capire.

${ }^{24}$ vulicipio : detto anche vulicèpi o battello di Valle Vulicipio (Valli di Comacchio/ Ferrara). Si tratta di una tipica imbarcazione lagunare dalla struttura leggera e dalla linea affusolata, costituita da fondo piatto, piuttosto stretto in rapporto alla lunghezza del natante. E leggermente arcuata alle estremità di prua e poppa.

${ }^{25}$ coso : qualsiasi oggetto o individuo di cui non si sappia il nome.

${ }^{26}$ fitta : sensazione acuta e dolorosa, avvertita all'improvviso.

27 immedesimandosi : identificandosi.

${ }^{28}$ dissanguato : privo di sangue.
} 
Aspettare, va bene : ma che cosa, e fino a quando ? Si sentiva la testa confusa, sbalordita ${ }^{29}$ : affollata di domande che non ricevevano risposta.

Passarono così molti minuti. Finché, improvvisamente, si rese conto che l'airone si era mosso.

$[\ldots]$

E finalmente se lo trovò faccia a faccia, ad un passo dalla botte, in procinto $\mathrm{di}^{30}$ prender terra. Una volta di più si era fermato. Color marrone in 60 tutto, tranne che nelle piume del collo e del petto, di un delicato tono bei$g e^{31}$, e tranne che nelle gambe d'un giallo-bruno da osso scarnificato ${ }^{32}$, da reliquia ${ }^{33}$, piegava leggermente la testa da una parte, osservandolo : incuriosito, sì, ma non spaventato. E lui, senza muoversi, senza quasi respirare (il sangue lo perdeva da uno squarcio a metà dell'ala, all'altezza 65 dell'articolazione), ebbe modo di ricambiare abbastanza a lungo quello sguardo...

(da GIORGIO BASSANI, L'airone, Milano, Mondadori, 1971)

\footnotetext{
${ }^{29}$ sbalordita: stordita, intontita dalla confusione.

${ }^{30}$ in procinto di: sul punto di.

${ }^{31}$ beige : colore tra il nocciola e l'avana.

32 scarnificato : senza carne.

${ }^{33}$ reliquia: ciò che resta del corpo, delle vesti o degli oggetti appartenuti a un santo o a un beato.
} 
1. Vero, Falso o Non Si Sa?

a) L'uccello volava in alto con estrema facilità.

\section{F NSS}

b) Il volo dell'airone non era agevolato dalla direzione in cui soffiava il vento.

c) Mentre si avvicinava ai due cacciatori, l'airone perdeva quota.

d) La cagna ha azzannato l'airone appena questi ha toccato terra.

e) Il protagonista si sentiva turbato perché non riusciva a capire le azioni di Gavino.
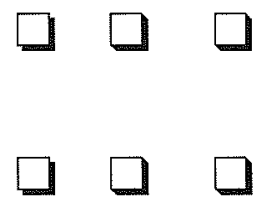

f) Gavino non ha sparato di nuovo perché ha avuto pietà dell'airone.
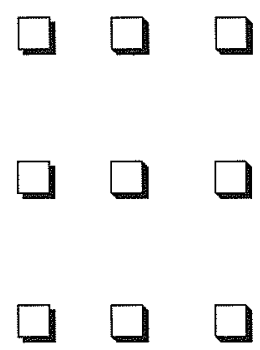

2. Combina i seguenti modi di dire con i relativi significati.

(I) a. essere con la corda al imporre gravi condizioni, profittando collo delle circostanze

b. prendere per il collo con frettolosa e spericolata velocità

c. piegare il collo trovarsi in guai seri

d. a rotta di collo dominare, opprimere una persona

e. stare sul collo a qualcuno sottomettersi, umiliarsi 
(II) a. vivere per la pancia

b. predicare il digiuno a pancia piena

c. grattarsi la pancia

d. tenersi la pancia per le risate

e. mettere su pancia

(III) a. abbassare le ali

b. avere le ali ai piedi

c. aprire le ali

d. in un batter d'ali

e. tarpare le ali

(IV) a. avere la testa fra le nuvole
b. mettere la testa a posto
credersi chissà chi
c. montarsi la testa
affrontare un problema con intelli- genza
d. avere qualcuno in testa essere distratto
e. usare la testa
mettere giudizio

acquistare credito, prestigio

togliere forza, capacità di agire

correre rapidamente

umiliarsi

pensare intensamente a una persona 
3. Testina (r.19), isolotto (r.26) e spiaggetta (r.43) sono nomi alterati. Forma il diminutivo delle seguenti parole tratte dal brano.

uccello (r.2), corpo (r.7), vento (r.11), bestia (r.13), cuore (r.1718), osso (r.18), pezzi (r.29), cagna (r.30), animale (r.41-42), cose (r.48), piedi (r.49).

4. Forma dei verbi dai seguenti aggettivi.

isolato (r.2), grandi (r.6), lungo (r.8), soleggiata (r.23), inquieto (r.36), scuro (r.43), minaccioso (r.44), confusa (r.53-54), sbalordita (r.54).

5. Specifica se i seguenti avverbi o locuzioni avverbiali sono di tempo, di luogo, di quantità o di modo.

subito (r.2), lentamente (r.3), piuttosto (r.6), molto (r.6), via (r.12), adagio (r.14), a perpendicolo (r.15), sopra (r.15), adesso (r.17), più (r.17), per di dietro (r.20), meglio (r.22-23), improvvisamente (r.23), lassì (r.25), quindi (r.26), poi (r.28), giù (r.28), appena (r.31), in qua e in là (r.32), a scatti (r.32), dietro (r.37), alla larga (r.42), di traverso (r.44), totalmente (r.47), a sufficienza (r.51), bene (r.53).

6. Cambia i due paragrafi (r.25-29 e r.30-34) dal singolare al plurale. 
7. Cambia gli ultimi due paragrafi (r.47-54 e r.55-56) dal passato al presente.

8. Riempi gli spazi vuoti di questo brano tratto da un altro romanzo di Bassani, Il giardino dei Finzi-Contini, con una delle tre proposte sottoelencate.

(1) anni sono passati da quel

pomeriggio di giugno ? Più di trenta.

(3), se chiudo gli occhi, Micòl Finzi-Contini è ancora là , affacciata al muro di (4) del suo giardino, che mi guarda e mi parla. Era poco più (5) una bambina, nel 1929, una (6) magra e bionda con grandi occhi chiari, magnetici. Io, un ragazzetto in (7) corti, molto borghese e molto vanitoso, che un piccolo guaio scolastico bastava a (8) nella disperazione più infantile. Ci fissavamo (9). Al di sopra di (10), il cielo era azzurro e compatto, un caldo cielo già estivo, senza la (11) nube. Niente (12) mutarlo, e niente l'ha mutato, infatti, almeno nella (13). “.................. (14), vuoi o non vuoi ?", incalzò Micòl.

"Mah ... non so ...", cominciai a dire, accennando al muro. "Mi sembra molto (15)".

" ..................(16) non hai visto bene", ribatté lei, impaziente. "Guarda là ..., e là ...., e là", e (17) il dito per farmi 
osservare. "C'è una (18) di tacche, e perfino un chiodo, quassú in (19). L' (20) io".

1 a) tanti

b) quanti

c) troppi

2 a) indimenticabile

b) vicino

c) remoto

3 a) anche

b) forse

c) tuttavia

4 a) cinta

b) recinto

c) barriera

5 a) da

b) che

c) chi

6 a) tredicenne

b) tredici

c) tredicesima

7 a) pantaloncini

b) calzoni

c) calzini

8 a) mettere

b) gettare

c) lanciare

9 a) ognuno

b) insieme

c) entrambi

10a) lei

b) Lei

c) sé

11a) minima

b) minore

c) piccola

12a) sarebbe potuto

b) sarebbe riuscito

c) avrebbe potuto

13a) mente

b) memoria

c) testa

14a) perché

b) perciò

c) allora

15a) alto

b) lungo

c) largo

16a) come

b) perché

c) $\mathrm{se}$

17a) puntava

b) indicava

c) mostrava

18a) specie

b) scala

c) quantità

19a) superficie

b) cima

c) apice

20a) ho inchiodato

b) ho messo

c) ho piantato 
9. Spunti per la produzione orale e scritta.

a. Una giornata trascorsa a contatto con la natura.

b. La caccia e la tutela del patrimonio faunistico.

c. Una storia raccontata da un uccello che ha avuto la sfortuna di essere ferito da un cacciatore.

d. I giovani e la consapevolezza della salvaguardia dell'ambiente.

e. Una vivace discussione tra un cacciatore e un ambientalista.

\section{OLTRE IL BRANO}

\section{La sillaba}

Prende il nome di sillaba un fonema o un gruppo di fonemi che si articola in modo distinto e autonomo, con una sola emissione di voce. La sillaba è pertanto la più piccola tra le combinazioni foniche in cui possono considerarsi divise le parole, e in cui effettivamente si dividono ogniqualvolta sia necessario farlo, alla fine di un rigo, per andare a capo.

Per formare una sillaba è sempre necessaria la presenza di una vocale. Le sillabe che terminano in vocale si dicono aperte o libere (per es. le quattro sillabe di te-le-fo-no) ; quelle che terminano con una consonante si dicono chiuse o implicate (per es. le prime tre di im-por-tan-za).

Le parole composte da un'unica sillaba, che può essere anche una sola vocale, si chiamano monosillabi (dal greco mónos 'uno solo'); quelle 
di più sillabe polisillabi (dal greco polýs 'molto'). I polisillabi si dividono a loro volta in bisillabi, trisillabi, quadrisillabi ecc.

\section{La divisione in sillabe}

In fin di riga non si possono spezzare le parole arbitrariamente, ma si deve conservare integra l'unità della sillaba : per questo è necessario conoscere e rispettare le norme che regolano la divisione in sillabe. Vediamole :

- una vocale iniziale di parola, seguita da una sola consonante, fa sillaba a sé : a-nima, e-resia, i-sola, o-livo, u-ranio ;

- le consonanti semplici (non rafforzate né unite con altre consonanti) fanno sillaba con la vocale che segue : vo-le-re-mo, li-mo-na-ta, se-re-ni-tà, no-ti-fi-ca-re ;

- le consonanti doppie si dividono tra le due sillabe : at-ter-rare, pez-zettino, am-mat-tire, os-ses-sione. In questo gruppo si può far rientrare anche -cq(u)- : ac-qua, ac-quisto, tac-qui, nac-que;

- gruppi di due o tre consonanti diverse tra loro fanno sillaba con la vocale seguente se possono venire a trovarsi in principio di parola : a-bra-sivo, ca-tra-me, pule-dro, mi-cro-bo, ma-gro ( in italiano abbiamo infatti parole che cominciano con br-:brano, brina; con tr-:treno, trave; con dr-: drastico, dritto; con cr-: cresta, crine ; con gr-: grasso, grotta). Allo stesso modo si comporta la cosiddetta $s$ impura, cioè la $s$ seguita da una o più consonanti : e-sclu-do, ma-sti-no, ve-spro ;

- nei gruppi di due o tre consonanti diverse tra loro che non possono trovarsi in principio di parola, la prima consonante va con la vocale precedente, l'altra o le altre con la vocale della sillaba che segue : a-rit-metica, tec-ni-ca, pal-ma, um-bro, para-dig-ma, sub-do-lo, per-pe-tra-re, anam-ne-si, im-por-to (non vi sono infatti parole italiane, a parte alcune 
che riproducono integralmente voci di altre lingue, comincianti per $t m$-, $c n-, l m-, m b r-, g m-, b d-, r p-, m n-, m p-)$;

- sono indivisibili i dittonghi e i trittonghi, mentre due vocali in iato possono essere divise : pau-sa ma pa-u-ra, pio-ve ma pi-o-lo, pian-ta ma vi$a$-le. Per eliminare ogni possibilità d'errore è sufficiente non andare mai a capo con una vocale, e dividere quindi in fin di riga pau-ra come pausa, pio-lo come pio-ve, via-le come pian-ta ;

- digrammi e trigrammi non si dividono mai : se-gno, de-gli, fa-scia, pesce, mi-glio.

L'apostrofo in fin di riga è ammesso, e viene anzi usato abitualmente da alcuni giornali. In generale si tende però a preferire una divisione sull'albero a una sull'-albero o, a maggior ragione, a una sullo-albero : infatti il tipo sul-l'albero ha il vantaggio, nei confronti del secondo tipo (sull'albero), di conservare l'integrità della sillaba, e, nei confronti del terzo tipo (sullo-albero), di rispettare l'uso normale e l'effettiva volontà di chi scrive.

(da M. DARDANO, P. TRIFONE, Grammatica italiana, Bologna, Zanichelli, 1988, pp.416-17)

\section{Opere di GIORGIO BASSANI (Bologna 1916)}

Cinque storie ferraresi, 1956 ; Gli occhiali d'oro, 1958, Il giardino dei Finzi-Contini, 1962 ; L'alba ai vetri, 1963 ; L'airone, 1968, L'odore del fieno, 1972 ; Il romanzo di Ferrara, 1974 ; Di là dal cuore, 1984. 


\section{0}

\section{E ALLE STECCHE DELLE PERSIANE GIÀ L'ALBA}

Il trapestio ${ }^{1}$ delle sei o sette persone sul pavimento di legno della camera ebbe finalmente un arresto ${ }^{2}$. Quelli che più s'erano avvicinati al letto dalla parte occupata, tra cui la donna, chiamarono ancora, quasi sottovoce, per un riguardo, "señora", señora", chinandosi. E il vecchio Olocati la scoperse $^{4}$. Gli occhi della signora, aperti, non lo guardarono, guardavano il nulla. Un orribile coagulo ${ }^{5}$ di sangue si era aggrumato ${ }^{6}$, ancor vivo, sui capelli grigi, dissolti, due fili di sangue le colavano $^{7}$ dalle narici ${ }^{8}$, le scendevano sulla bocca semiaperta. Gli occhi erano dischiusi, la guancia destra tumefatta, la pelle lacerata ${ }^{9}$, e anche sotto l'orbita ${ }^{10}$, orribile. Le due povere mani levate ${ }^{11}$, scheletrite ${ }^{12}$, parevano protese verso "gli altri" come in una difesa

\footnotetext{
1 trapestio : trepestio, rumore fatto dai passi.

2 arresto : interruzione.

3 señora: "signora" in spagnolo.

${ }_{5}^{4}$ scoperse : scoprì.

${ }^{5}$ coagulo: risultato di una parziale solidificazione.

${ }^{6}$ aggrumato : raccolto in una piccola massa densa e soda.

${ }^{7}$ colavano: scendevano.

${ }^{8}$ narici: le due cavità che fanno comunicare le fosse nasali con l'esterno.

${ }^{9}$ lacerata: strappata.

10 orbita : ciascuna delle cavità in cui ruotano gli occhi.

11 levate : alzate.

${ }^{12}$ scheletrite : ridotte, per la magrezza, a pelle e ossa.
} 
$\mathrm{o}$ in una implorazione estrema. Esse poi apparivano graffiate $^{13}:$ macchie e sbavature $^{14}$ di sangue erano sul guanciale e sul lembo ${ }^{15}$ del lenzuolo.

Si accorsero che respirava, che solo le mani erano così, quasi fredde: $\operatorname{tardo}^{16}$, debolissimo, il polso batteva ancora. Allora fu subito 15 mandato per $^{17}$ medico, fu Bruno che corse. In paese già lo avevano svegliato, quasi a un presagio.

Egli finalmente arrivò, passando dal cancello grande di legno e dalla scaletta esterna : una trentina di persone erano state messe fuori della porta dal cugino dell'alcade ${ }^{18}$, poi dall'alcade, sopravvenuto lui pure, e sostavano sul terrazzo, confabulando ${ }^{19}$, rabbrividendo. Nessuno trovava la chiave del cancelletto di ferro. In casa erano rimaste la Peppa, la Beppina, la donna del cimitero, autorizzate a rendersi utili come potevano : e alcuni uomini, degli "aventi diritto".

Il vecchio medico di Lukones $^{20}$ in quelle tristissime contingenze ${ }^{21}$ si 25 rese molto utile. Aveva una barba di quattro giorni sulle guance cascanti ${ }^{22}$, non bianca ancora del tutto, ed era senza cravatta, con un colletto d'amido ${ }^{23}$ sfilacciato $^{24}$ e un po" "foeudra de salamm ${ }^{25}$ ", con occhi arrossati come per

\footnotetext{
13 graffiate : con la pelle lacerata da unghie.

14 sbavature : emissioni di bava, saliva che cola dalla bocca.

15 lembo : estremità.

16 tardo : lento.

17 mandato per : chiamato.

${ }^{18}$ alcade : variante arcaica di alcalde, primo magistrato nelle città spagnole o dell'America latina.

${ }^{19}$ confabulando: conversando in modo da non essere sentiti.

${ }^{20}$ Lukones : villaggio di un immaginario paese sudamericano di lingua spagnola con sovrimpressioni brianzole (la Brianza è una zona della Lombardia).

${ }^{21}$ contingenze: circostanze.

22 cascanti : flosce perché prive di energia, a causa dell'età.

23 amido : un composto chimico in forma di granuli o di polvere bianchissima che si applica per dare consistenza alla biancheria mentre si stira.

${ }^{24}$ sfilacciato : ridotto in cattive condizioni.

${ }^{25}$ foeudra de salamn: in milanese, significa "buccia di salame".
} 
una blefarite ${ }^{26}$, stanchi, gonfi e piccoli dalla fatica e dal sonno : sotto ai due piccoli bulbi ${ }^{27}$ le occhiaie ${ }^{28}$ gonfie, a lùnula ${ }^{29}$, parevano due amache ${ }^{30}$ o due ghirbe $^{31}$. Aveva portato con sé il prevedibile nella sua borsa nera e bisunta ${ }^{32}$ che tutti conoscevano, rifornita ad istinto, come da lunghi anni la praticaccia omnibus $^{33}$ gli aveva suggerito, e poi a mano a mano corretto i suggerimenti con le novità sempre più perfette del pronto soccorso. La depose sul tavolino in un angolo. Altri impicci ${ }^{34}$ e bende aveva affidato al Bruno, che pure ve li depose. Il dottore si accostò al letto, guardò quell'essere immobile e così orrendamente offeso : "così l'avete trovata ?", disse, prese la mano e distese quasi con una certa fatica il braccio scheletrito che i pizzi ${ }^{35}$ della camicia da notte ricadendo avevano lasciato emergere nell'implorazione e nella difesa, l'una e l'altra vane. Tastò il polso destro mentre con l'altra mano ricompose distendendolo l'altro braccio della povera indifesa. $\mathrm{Si}$ chinò ad ascoltare il cuore, poi nuovamente con lo stetoscopio ${ }^{36}$.

$$
[\ldots]
$$

Si comprese da tutti, al riscontrare delle tracce di sangue sullo spigolo ${ }^{37}$ del tavolino da notte, verso il letto, che il capo così ferito doveva avervi battuto violentemente ; forse qualcuno doveva averla afferrata a due mani, pel ${ }^{38}$ collo, e averle sbattuto il capo contro lo spigolo del tavolino da

\footnotetext{
${ }^{26}$ blefarite : infiammazione del bordo delle palpebre, che si manifesta con arrossamento, caduta delle ciglia e formazione di piccole squame.

${ }^{27}$ bulbi: le parti tondeggianti degli occhi.

${ }^{28}$ occhiaie: infossamenti o macchie livide sotto gli occhi.

${ }^{29}$ Iunula : a forma di falce di luna.

${ }^{30}$ amache : reti o tele fissate a due pali o tronchi d'albero che servono per il riposo.

31 ghirbe : sacchi di tela impermeabile per portare acqua.

${ }^{32}$ bisunta: unta e sporca.

33 omnibus : per tutti e in tutti i casi.

${ }^{34}$ impicci : impedimenti.

${ }_{35}^{35}$ pizzi : trine da bordura col margine libero smerlato.

${ }^{36}$ stetoscopio: strumento per rilevare e amplificare i battiti del cuore.

${ }^{37}$ spigolo : parte angolare prominente di costruzioni, oggetti o simili.

${ }^{38}$ pel : per il.
} 
notte, per terrorizzarla, o deliberato ad ucciderla. Terribile fu e permaneva a tutti l'aspetto di quel volto ingiuriato ${ }^{39}$, ch'essi conoscevano così nobile e buono pur nel disfacimento ${ }^{40}$ della vecchiezza.

"Lasciamola tranquilla" disse il dottore, "andate, uscite".

Nella stanchezza senza soccorso in cui il povero volto si dovette raccogliere tumefatto, come in un estremo recupero della sua dignità, parve a tutti di leggere la parola terribile della morte e la sovrana coscienza della impossibilità di dire : Io.

L'ausilio $^{41}$ dell'arte medica, lenimento ${ }^{42}$, pezzuole ${ }^{43}$, dissimulo ${ }^{44}$ in parte l'orrore. Si udiva il residuo ${ }^{45}$ d'acqua e alcool dalle pezzuole strizzate $^{46}$ ricadere gocciolando in una bacinella. E alle stecche ${ }^{47}$ delle persiane $^{48}$ già l'alba. Il gallo, improvvisamente, la suscitò dai monti lontani, perentorio $^{49}$ ed ignaro ${ }^{50}$, come ogni volta. La invitava ad accedere e ad elencare $\mathrm{i}$ gels $\mathrm{s}^{51}$, nella solitudine della campagna apparita ${ }^{52}$.

(da CARLO EMILIO GADDA, La cognizione del dolore, Milano, Garzanti, 1999)

\footnotetext{
${ }^{39}$ ingiuriato : danneggiato, deformato, oltraggiato.

40 disfacimento : rovina.

41 ausilio : aiuto.

42 lenimento: conforto.

${ }^{43}$ pezzuole : quadrati di stoffa di cotone.

44 dissimulò : nascose, mascherò.

45 residuo: ciò che rimaneva.

${ }^{46}$ strizzate : strette fortemente, facendo uscire tutto il liquido.

${ }^{47}$ stecche: asticelle di legno, sottili e lunghe.

${ }^{48}$ persiane: imposte esterne di finestre, che attenuano la luce ma lasciano passare 1 'aria.

${ }^{49}$ perentorio: che non ammette rifiuto o rinvio.

50 ignaro: inconsapevole.

51 gelsi : piante con foglie dentate di cui si nutrono i bachi da seta.

52 apparita : forma rara, apparsa.
} 
1. Vero, Falso, o Non Si Sa?

\section{F NSS}

a. La donna era stata ferita a morte da un ladro.

b. La donna fu trovata per terra e messa sul letto dai suoi conoscenti.

c. Il vecchio medico aveva un aspetto trasandato perché viveva da solo.

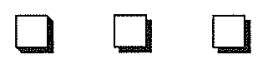

d. Quando l'hanno trovata, l'anziana era ancora viva.

e. C'erano tracce di sangue sullo spigolo del tavolino da notte, perché la donna ci aveva battuto la testa.

f. Da giovane la donna era stata molto bella.

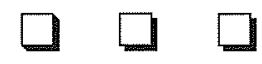

g. Nella sua vecchiaia, la donna aveva perso i lineamenti nobili del viso.
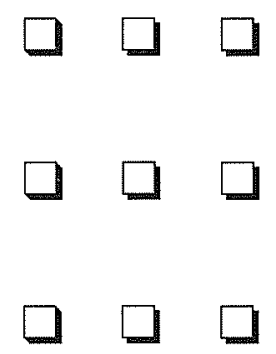

2. Indica il significato delle seguenti parole o espressioni scegliendo la risposta corretta o più appropriata tra le tre proposte.

a. tumefatta (r.8-9) : (I) paffuta ; (II) gonfia ; (II) pallida.

b. in una implorazione estrema (r.10-11) : (I) in un'ultima supplica ; (II) in un gesto d'impazienza; (III) in una richiesta di aiuto.

c. a un presagio (r.15-16) : (I) per comodità ; (II) per sicurezza ; (III) per un presentimento. 
d. confabulando (r.20) : (I) sentendo freddo; (II) raccontando delle favole ; (III) discorrendo sottovoce.

e. sfilacciato (r.27) : (I) che si chiude con dei lacci ; (II) di filo molto fine ; (III) sciupato.

f. deliberato (r.47) : (I) deciso ; (II) libero ; (III) costretto.

g. ingiuriato (r.48) : (I) brutto ; (II) strano ; (III) deturpato.

h. bacinella (r.58) : (I) piccolo vaso ; (II) piccolo recipiente per usi domestici ; (III) piccolo vassoio.

3. Specifica se i participi passati sottoelencati sono usati nel testo come aggettivi, sostantivi, o nei tempi composti, e fornisci anche l'infinito di ciascun participio passato.
a. avvicinati (r.2)
b. occupata (r.3)
c. aperti (r.5)
d. aggrumato (r.6)
e. dissolti (r.7)
f. dischiusi (r.8)
g. tumefatta (r.8-9)
h. difesa (r.10-11)
i. arrossati (r.27)
1. soccorso (r.33)
m. ferito (r.44) 
4. Riempi gli spazi vuoti con preposizioni semplici o articolate.

Carlo Emilio Gadda nacque Milano 1893 famiglia borghese; prese parte ......... prima guerra mondiale e . questa drammatica esperienza nacque il Giornale di guerra e di prigionia, pubblicato molti anni più tardi. Laureato .......... ingegneria, esercitò ......... qualche anno la professione ......... Italia e estero. ......... frattempo aveva iniziato la collaborazione . "Solaria", la rivista che, edita ......... Firenze, ......... il 1926 e il 1936, si impegnò, ......... evidente polemica ......... il nazionalismo culturale fascista, in un fecondo dialogo ......... la cultura europea, costituendo un punto di aggregazione ......... la più giovane letteratura italiana. Per le edizioni di "Solaria" Gadda pubblicò le sue prime opere narrative e, dedicatosi esclusivamente ......... letteratura, ......... partire ......... anni quaranta, stampò una serie opere che lo qualificano come uno .......... maggiori scrittori italiani del Novecento [...].

Il suo nome è legato .......... una profonda azione ......... rinnovamento ......... narrativa italiana, sia ......... il rifiuto ......... struttura ......... romanzo tradizionale , ......... favore .......... una narrazione imprevedibilmente aperta ......... intervento ironico o ferocemente sarcastico ......... autore, sia ......... la sua capacità scrivere .......... una prosa composita, quale l'uso 
dialetti diversi, gerghi, linguaggi tecnico-scientifici, arcaismi e neologismi conferiscono una eccezionale vitalità stilistica.

(da W. BINNI, E. GHIDETTI, F. ANDREOLI, Immagini del reale, Palermo, Palumbo, 1984, p.655)

5. Specifica se il complemento introdotto dalla preposizione di indica causa, materia, specificazione o tempo.
a. pavimento di legno (r.1)
b. coagulo di sangue (r.6)
c. lembo del lenzuolo (r.12)
d. cugino dell'alcade (r.19)
e. la chiave del cancelletto (r.20-21)
f. cancelletto di ferro (r.21)
g. la donna del cimitero (r.21-22)
h. una barba di quattro giorni (r.25)
i. i pizzi della camicia da notte (r.37-38)
1. l'altro braccio della povera indifesa (r.40)
m. spigolo del tavolino (r.43-44)
n. disfacimento della vecchiezza (r.49)
o. la parola terribile della morte (r.54)
p. il residuo d'acqua e alcool (r.57)
q. solitudine della campagna (r.61) 
6. Volgi in forma esplicita, usando il modo indicativo, le seguenti proposizioni implicite. (es. : Chiamarono per nome la madre, il figlio, gridando, verso le finestre del $1^{\circ}$ piano/ chiamarono per nome la madre, il figlio, mentre gridavano, verso le finestre del $1^{\circ}$ piano)

a. chinandosi (r.4)

b. sopravvenuto lui pure (r.19)

c. confabulando, rabbrividendo (r.20)

d. ricadendo (r.38)

e. distendendolo (r.40)

f. al riscontrare delle tracce di sangue sullo spigolo del tavolino da notte, verso il letto (r.43-44)

g. gocciolando (r.58)

7. Spunti per la produzione orale e scritta.

a. Scrivi un articolo per un quotidiano su un episodio di cronaca nera.

b. Riassumi e commenta il brano.

c. Gli anziani e la solitudine.

d. In che modo la società può aiutare gli anziani a non sentirsi emarginati?

e. Clonazione, trapianto, eutanasia. Implicazioni morali, etiche e religiose.

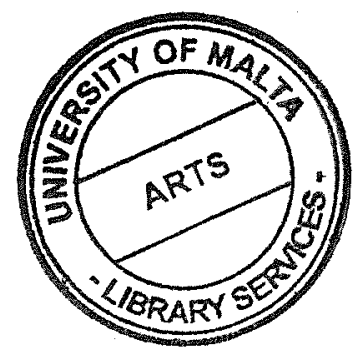




\section{OLTRE IL BRANO}

\section{I nomi che resistono : esatti e belli}

"Lasciamola tranquilla" disse il dottore, "andate, uscite".

Nella stanchezza senza soccorso in cui il povero volto si dovette raccogliere tumefatto, come in un estremo recupero della sua dignità, parve a tutti di leggere la parola terribile della morte e la sovrana coscienza della impossibilità di dire: Io.

L'ausilio dell'arte medica, lenimento, pezzuole, dissimulò in parte l'orrore. Si udiva il residuo d'acqua e alcool dalle pezzuole strizzate ricadere gocciolando in una bacinella. E alle stecche delle persiane già l'alba. Il gallo, improvvisamente, la suscitò dai monti lontani, perentorio ed ignaro, come ogni volta. La invitava ad accedere e ad elencare i gelsi, nella solitudine della campagna apparita.

[...] Questi sarebbero per così dire le ultime righe de La cognizione del dolore, romanzo mai finito.

La situazione è quella, in due parole : c'è una vecchia signora che è stata aggredita di notte, e sta per morire. Non si sa bene se morirà. Non lo sapremo mai. Hanno passato la notte, quel che restava della notte a cercare di curarla. Adesso lei è stesa nel suo letto. Ormai quello che si poteva fare, l'hanno fatto. È un'agonia, silenziosa, in una stanza. Questo accade nell'ultimo brandello di notte. Ciò che accade dopo è che viene l'alba. Tutto qua !

Aveva da raccontare questo, Gadda. E quello che scrisse fu questo : "Lasciamola tranquilla", disse il dottore, "andate, uscite". Punto, a capo.

Nella stanchezza senza soccorso in cui il povero volto si dovette raccogliere tumefatto, come in un estremo ricupero della sua dignità, parve a tutti di leggere la parola terribile della morte e la sovrana coscienza della impossibilità di dire, due punti, Io, con la i maiuscola. 
Quindi c'è una cosa che noi, molti di noi conoscono, quando tu vedi un agonizzante, uno che sta per morire, e, come dice lui, no, nei malati si cerca poi di riordinare un po' questo disastro accaduto, ma loro comunque hanno sempre tutti i capelli sudati, e poi il pigiama sempre storto, la dentiera in giro. Fanno fatica a tenere insieme questo corpo che sta per morire, no ? E mentre tu lo guardi, in quel momento pensi. C'è una parte di te che lo pensa. Dice: "Ma, certo, che se poi tutto finisce così, e allora veramente, cosa si salva di quello che io ho fatto ? Di quello che quest'uomo ha fatto? Se poi bisogna finir con la dentiera di traverso, questi capelli col cuscino. Perché facciamo tutto questo ? Perché quella volta mi sono incazzato ? Perché poi ho amato tanto ? E poi tanto !"

Questa cosa vagamente, noi la pensiamo, naturalmente. A saperla dire in una riga, con esattezza! Questa cosa è questa : parve a tutti di leggere, sul volto, come fosse un libro, la parola terribile della morte. È una parola che usiamo tante volte, ma è lì, la leggi, terribile ! E la sovrana coscienza della impossibilità di dire: Io. E per quanto ti arrangi a pensare che ... Punto. A capo.

L'ausilio dell'arte medica, lenimento, pezzuole, dissimulò in parte l'orrore. Si udiva il residuo d'acqua e alcool dalle pezzuole strizzate ricadere gocciolando in una bacinella.

Questa è un'inquadratura cinematografica molto stretta. E lui te la rende come un suono: Si udiva. Ma in realtà quello che sta facendo, è, ti sta facendo vedere : Si udiva. Inquadratura stretta : una bacinella, ci sono i panni con cui l'hanno medicata, che li hanno strizzati, ma ancora tac, tac, tac. Un rumore, un'immagine, molto piccola, anche insignificante, nella stanza di uno che sta per morire. Una bacinella!

E alle stecche delle persiane già l'alba. Punto. C'era da dire che stava per cominciare il giorno. E allora, cinema : alzo e guardo le persiane. 
E io avrei scritto: "E alle persiane l'alba". Ma Gadda era esatto: E alle stecche delle persiane già l'alba. E non c'è verbo. Non che "c'è l'alba", "ce sta l'alba" ... la televisione, no ! Non c'è bisogno del verbo. L'alba! Ed effettivamente quello che tu vedi è nelle stecche delle persiane, il rimbalzo della luce. Più sei esatto, più quel nome resisterà. Se tu sei vago, sono nomi che durano una sera. Il giorno dopo non sai cosa farti. Ma quello, tac !

Mancano due frasi. E quello che doveva dire qui Gadda era semplicemente: "l'alba", che è anche un luogo comune, neanche difficile, l'han già detto in molti. Migliaia di persone l'hanno già detto, scritto. Ma quello pensò di fare in quelle ultime due frasi. E lui scrisse questo : il gallo, che voglio dire, se vi chiedo la cosa più banale, la cosa più lisa, inservibile, che vi viene in mente, legata all'alba, voi dite : "il gallo". Quello, basta, non lo userà. Il gallo! Che si va a mettere nei pasticci da sé. Acrobazie, no ? Sono tutti buoni. Uso il gallo, tac! Il gallo, improvvisamente, la suscitò dai monti lontani, perentorio ed ignaro, come ogni volta. E il gallo è banale, ma che lui, l'alba, la susciti, ah! Non è che canta, la chiama. C'è un verbo per quella cosa, c'è un verbo nella nostra lingua, c'è un verbo. Che dice quella cosa, ha il suono di quella cosa. Ed è il nome di quella cosa : "suscitare", tac ! Dopo di lui era ben impossibile far una scena con un gallo, questo sì. Lui, l'ultimo. Almeno per noi italiani! Il gallo, improvvisamente, la suscitò dai monti lontani, perentorio ed ignaro, come ogni volta. Punto.

Ultima frase. Come nei temi che l'ultima frase cerchiamo di farla un po' più bella, no ? Non ho mai capito perché. Per lasciare una buona impressione, dici, magari guadagno sei, mah! Però anche lui, anche Gadda, cioè lui, anche lui, l'ultima frase è bella. Quindi non era stupido, eh! Il gallo, improvvisamente, la suscitò. Punto. La invitava ad accedere e ad elencare $i$ gelsi, nella solitudine della campagna apparita. Ah ! La invitava : tutta una storia di corteggiamento, tu vedi il gallo elegante, la signora, prego, lei che 
arriva. Ad accedere. Perché lei non "arriva", non "ce sta", ma neanche "arriva", nemmeno "si avvicina". Accede : come Catherine Deneuve. Non è che "cammina", Catherine Deneuve. No. Vuol dire esattamente cosa fa, è un'altra cosa. E l'alba arriva e accede. La invitava ad accedere. C'è questo clima veramente da festa, da corteggiamento, d'alto bordo. E poi Gadda, è solo lui. La invitava ad accedere e ad elencare i gelsi. Questo è pazzesco. Elencare i gelsi. Si possono veramente elencare i gesti, le parole, veramente. Ma voi lo immaginate questo viale di gelsi, no ? È l'alba che arriva, ed è luce. Che arriva. E prima era buio, arriva la luce. Il primo gelso, secondo gelso, terzo gelso. Trr ... e sale. E intanto elenca. La luce, è quello che fa. Nella vita, la luce elenca. Prima non capisci, dopo di che ... elenco. Come elenco dei lavori fatti dal meccanico : questo, quello, il faro, il fanalino. Tu sai finalmente cosa ha fatto. Arriva la luce e tu sai quello che fai, elencare. E guarda, in 3 parole : c'è un movimento, c'è il senso di un gesto, il senso della luce. Per sempre, bloccato lì. Elencare $i$ gelsi. Gelsi che ormai abbiamo visto e nascono in questa frase. Per noi. Solo per dirci cose rare. Virgola. Nella solitudine della campagna apparita. Qui c'è una cosa di tempo, molto bella. Perché lui inizia la frase, questa è una frase, niente più che una frase. Inizia la frase che l'alba arriva. Elenca i gelsi, e la finisce nella solitudine della campagna apparita. Fop, è arrivata ! Nel tempo di una frase, tu vedi l'inizio e poi ti guardi intorno : è arrivata. Elenca, trac, luce. Spazi, il tempo, tutto in una frase. Era molto esatto, Gadda. Non sarebbe il grande che è se non avesse avuto anche un senso della bellezza, grandissimo. Perché i nomi che resistono, sono esatti e belli. Perché quelli esatti e basta, restano nell'università. A noi servono abbastanza poco. E così quest'ultima frase, molto italiana, è una melodia, è un canto. È musica, questa qui. Oltre ad essere precisa, esatta, è musica. La invitava ad accedere e ad elencare i gelsi. Non sentite niente? Tipo: truffa, propano, propellente, no ? È rumore, ru- 
more. È la danza dell'alba, questa. Qui, tutti gli altri nel mondo, traduzioni, mai salveranno questo. Solo noi. Noi possiamo sentire. La invitava ad accedere e ad elencare $i$ gelsi, nella solitudine della campagna apparita. Non trovate uno spigolo, potete stare tutta una vita, non trovate uno spigolo. Noi abbiamo una bella lingua, l'italiano è una bellissima lingua. Lui la usava da Dio.

E poi è canto. La invitava ad accedere e ad elencare i gelsi, nella solitudine della campagna apparita. Chiunque di noi sente che quella è la musica giusta, ritmo e melodia. $\mathrm{Na}$, na, ta, tac, tac, tac. Ultima frase del testo. Nome, tac! Quelli nominano, noi raccontiamo storie.

(ALESSANDRO BARICCO. Dal programma televisivo di RAIDUE "Totem" del 28 dicembre 1998)

\section{Opere di CARLO EMILIO GADDA (Milano 1893 - Roma 1973)}

La Madonna dei filosofi, 1931 ; L'Adalgisa, 1944 ; Giornale di guerra e di prigionia, 1955 ; Quer pasticciaccio brutto de via Merulana, 1957 ; Accoppiamenti giudiziosi, 1963 ; La cognizione del dolore, 1963 ; Eros e Priapo , 1967 ; La meccanica, 1970 ; Novella seconda, 1971. 


\section{1 \\ LA VERNA}

21 Settembre (presso la Verna ${ }^{I}$ )

Io vidi dalle solitudini mistiche staccarsi una tortora ${ }^{2}$ e volare diste$\mathrm{sa}^{3}$ verso le valli immensamente aperte. Il paesaggio cristiano segnato di 5 croci inclinate dal vento ne fu vivificato misteriosamente. Volava senza fine sull'ali ${ }^{4}$ distese leggera come una barca sul mare. Addio colomba, addio ! Le altissime colonne di roccia della Verna si levavano a picco ${ }^{5}$ grige nel crepuscolo ${ }^{6}$, tutt'intorno rinchiuse dalla foresta cupa.

Incantevolmente cristiana fu l'ospitalità dei contadini là presso?

10 Sudato mi offersero acqua. "In un'ora arriverete ${ }^{8}$ alla Verna, se Dio vole". Una ragazzina mi guardava cogli occhi neri un po' tristi, attonita ${ }^{10}$ sotto

\footnotetext{
${ }^{1}$ La Verna : $45 \mathrm{~km}$ a Nord di Arezzo in Toscana, a 1128 metri di altezza, sorge un convento nel cuore di un'antica foresta. Fu fondato nel 1214 da San Francesco d'Assisi sull'omonimo monte dove nel 1224 ricevette le stimmate (stigmate), cioè la riproduzione delle piaghe di Cristo sul suo corpo.

${ }^{2}$ tortora : piccolo uccello dei Colombiformi, con la testa di color cenere, le parti dorsali grige e castane a macchie allungate nere e la coda grigia-nerastra.

3 distesa: spiegata, allargata.

${ }^{4}$ sull'ali : sulle ali.

5 a picco: a perpendicolo, con direzione verticale.

${ }^{6}$ crepuscolo: la luce che precede l'alba o, come in questo caso, segue il tramonto. ${ }_{8}^{7}$ presso: nelle vicinanze.

8 arriverete : arriverà. Nelle campagne umbre e toscane si usa ancora il voi invece della forma di cortesia Lei.

${ }^{9}$ vole : vuole.

${ }^{10}$ attonita : stupita, sbalordita.
} 
l'ampio cappello di paglia. In tutti un raccoglimento ${ }^{11}$ inconscio, una serenità conventuale ${ }^{12}$ addolciva a tutti $\mathrm{i}$ tratti $^{13}$ del volto. Ricorderò per molto tempo ancora la ragazzina e i suoi occhi conscii ${ }^{14}$ e tranquilli sotto il cappel15 lone monacale.

Sulle stoppie ${ }^{15}$ interminabili sempre più alte si alzavano le torri naturali di roccia che reggevano ${ }^{16}$ la casetta conventuale rilucente di dardi ${ }^{17}$ di luce nei vetri occidui ${ }^{18}$.

$$
[\ldots]
$$

$$
[\ldots]
$$

Antri $^{19}$ profondi, fessure ${ }^{20}$ rocciose dove una scaletta di pietra si sprofonda in un'ombra senza memoria, ripidi colossali bassorilievi ${ }^{21}$ di colonne nel vivo sasso: e nella chiesa $l^{1}$ angiolo ${ }^{22}$, purità dolce che il giglio ${ }^{23}$ divide e la Vergine eletta, e un cirro ${ }^{24}$ azzurreggia ${ }^{25}$ nel cielo e un'anfora ${ }^{26}$

\footnotetext{
11 raccoglimento : concentrazione spirituale.

12 conventuale: da convento, da monastero.

13 tratti : lineamenti.

${ }^{14}$ conscii : consci.

${ }^{15}$ stoppie : i residui degli steli e delle paglie che rimangono in un campo dopo la mietitura.

${ }^{16}$ reggevano: sostenevano.

${ }^{17}$ dardi : raggi.

18 occidui : posti a occidente.

19 antri : caverne.

${ }^{20}$ fessure : aperture lunghe e sottili.

${ }^{21}$ bassorilievi : sculture nelle quali le figure emergono dal fondo con poco stacco.

22 angiolo: angelo.

${ }^{23}$ giglio : fiore bianco e profumatissimo che simboleggia la purezza.

24 cirro: nuvoletta bianca, situata a grande altezza.

25 azzurreggia : assume a poco a poco il color azzurro.

${ }^{26}$ anfora: vaso a due manichi, più stretto all'imboccatura e al piede.
} 
classica rinchiude la terra ed i gigli : che appare nello scorcio $^{27}$ giusto $^{28}$ in cui appare il sogno, e nella nuvola bianca della sua bellezza che posa un istante il ginocchio a terra, lassù così presso al cielo $[\ldots]$

Il corridoio, alitato ${ }^{29}$ dal gelo degli antri, si veste tutto della leggen30 da Francescana ${ }^{30}$. Il santo appare come l'ombra di Cristo, rassegnata, nata in terra d'umanesimo ${ }^{31}$, che accetta il suo destino nella solitudine. La sua rinuncia è semplice e dolce : dalla sua solitudine intona il canto alla natura con fede : Frate Sole, Suor Acqua, Frate Lupo. Un caro santo italiano. Ora hanno rivestito la sua cappella scavata nella viva roccia. Corre tutt'intorno

35 un tavolato ${ }^{32}$ di noce dove con malinconia potente un frate ... da Bibbiena ${ }^{33}$ intarsiò ${ }^{34}$ mezze figure di santi monaci. La semplicità bizzarra del disegno bianco risalta quando l'oro del tramonto tenta versarsi dall'invetriata ${ }^{35}$ prossima nella penombra ${ }^{36}$ della cappella. Acquistano allora quei sommarii ${ }^{37}$ disegni un fascino bizzarro e nostalgico. Bianchi sul tono ricco del noce

${ }^{27}$ scorcio : rappresentazione di un oggetto le cui parti sono disposte su un piano obliquo a chi guarda.

${ }^{28}$ giusto: preciso.

${ }^{29}$ alitato: soffiato, cioè in cui c'era il soffio del gelo degli antri.

${ }^{30}$ Francescana : francescana.

31 terra d'umanesimo : terra di scoperta di valori umani e storici.

${ }^{32}$ tavolato : un insieme di tavole connesse, cioè di assi rettangolari di legno, lunghe e strette.

${ }^{33}$ Bibbiena : comune del Casentino, in provincia di Arezzo.

${ }^{34}$ intarsiò : inserì a incastro pezzi di legno a vari colori in modo da formare figure o disegni ; intarsiare si usa anche per indicare l'incastro di madreperla, tartaruga, metalli ecc. su legno.

${ }^{35}$ invetriata : lastra o insieme di lastre di vetro che occupano il vano di una finestra o di una porta.

${ }^{36}$ penombra: condizione intermedia tra l'ombra e la luce.

${ }^{37}$ sommarii : sommari, fatti in modo semplice ed essenziale. 
40 sembrano rilevarsi ${ }^{38}$ i profili ieratici ${ }^{39}$ dal breve paesaggio claustrale da cui sorgono decollati ${ }^{40}$, figure di una santità fatta spirito, linee rigide enigmatiche di grandi anime ignote. Un frate decrepito nella tarda ora si trascina nella penombra dell'altare, silenzioso nel saio $^{41}$ villoso $^{42}$, e prega le preghiere d'ottanta anni d'amore. Fuori il tramonto s'intorbida ${ }^{43}$. Strie ${ }^{44}$ minacciose 45 di ferro si gravano ${ }^{45}$ sui monti prospicenti ${ }^{46}$ lontane. Il sogno è al termine $\mathrm{e}$ l'anima improvvisamente sola cerca un appoggio una fede nella triste ora. Lontano si vedono lentamente sommergersi ${ }^{47}$ le vedette ${ }^{48}$ mistiche e guerriere dei castelli del Casentino ${ }^{49}$. Intorno è un grande silenzio un grande vuoto nella luce falsa dai freddi bagliori ${ }^{50}$ che ancora guizza ${ }^{51}$ sotto le strette della 50 penombra. E corre la memoria ancora alle signore gentili dalle bianche braccia ai balconi laggiù : come in un sogno : come in un sogno cavalleresco!

Esco : il piazzale è deserto. Seggo ${ }^{52}$ sul muricciolo ${ }^{53}$. Figure vagano, facelle ${ }^{54}$ vagano e si spengono: $i$ frati si congedano dai pellegrini. Un alito continuo e leggero soffia dalla selva in alto, ma non si ode né il fru-

\footnotetext{
${ }^{38}$ rilevarsi : sollevarsi.

${ }^{39}$ ieratici : improntati a solenne compostezza e gravità, sacri, sacerdotali.

${ }^{40}$ decollati : staccati dal collo.

41 saio : tonaca monacale confezionata con panno rozzo.

42 villoso : coperto da un manto di pelo.

43 s'intorbida: s'offusca.

${ }^{44}$ strie : righe sottili che spiccano su una superficie di colore diverso.

45 si gravano: si appoggiano pesantemente.

${ }^{46}$ prospicenti: prospicienti, volti verso un luogo.

47 sommergersi : affondare, immergersi.

48 vedette : le parti più elevate da cui si può osservare il territorio circostante.

${ }^{49}$ Casentino : zona della provincia di Arezzo, in Toscana.

${ }^{50}$ bagliori : luci improvvise che abbagliano.

51 guizza : scatta.

52 seggo : siedo (sedere).

53 muricciolo: muro basso che serve a delimitare una proprietà.

${ }^{54}$ facelle: piccole ma intense luci.
} 
sciare della massa oscura né il suo fluire ${ }^{55}$ per gli antri. Una campana dalla chiesetta francescana tintinna nella tristezza del chiostro : e pare il giorno dall'ombra, il giorno piagner ${ }^{56}$ che si muore ${ }^{57}$.

(da DINO CAMPANA, Canti orfici, Milano, Marcos y Marcos, 1989)

55 fluire : scorrere.

${ }^{56}$ piagner : piangere.

${ }^{57}$ si muore : finisce. 
1. Scelta multipla. Una delle tre alternative è sbagliata. Segna le due risposte giuste.

a. Il volo della tortora

(I) rendeva vivo il paesaggio

(II) creava un' atmosfera d'incanto

(III) era impedito dal vento che soffiava

b. Gli abitanti della zona

(I) sembravano diffidenti

(II) erano molto ospitali

(III) rispecchiavano l'ambiente sereno

c. Il convento si trovava

(I) vicino ad una casetta

(II) sulle rocce

(III) sopra campi coltivati a cereali

d. La cappella originale di S. Francesco è stata poi

(I) decorata con legno intarsiato

(II) coperta di legno di noce

(III) rivestita

e. Gli intarsi delle pareti

(I) si possono apprezzare maggiormente nella luce del tramonto

(II) creano un'atmosfera grave e solenne

(III) riportano episodi della vita di un frate molto vecchio

f. All'ora del tramonto

(I) i frati si chiudono nel convento 
(II) i pellegrini entrano nella chiesetta francescana

(III) si sente suonare la campana del convento

2. Trova nel testo gli aggettivi che hanno il significato di:

a) caratterizzate da profonda spiritualità ( $1^{\circ}$ paragrafo)

b) buia $\left(1^{\circ}\right.$ paragrafo $)$

c) caratterizzato da una semplicità austera $\left(2^{\circ}\right.$ paragrafo $)$

d) battuti dai raggi del sole che volge al tramonto ( $3^{\circ}$ paragrafo)

e) che si riferisce al chiostro ( $5^{\circ}$ paragrafo)

f) decapitati $\left(5^{\circ}\right.$ paragrafo)

g) che sono volti verso un luogo $\left(5^{\circ}\right.$ paragrafo)

h) nobile, da cavaliere $\left(5^{\circ}\right.$ paragrafo)

3. La parola bassorilievi (r.23) è un nome composto. Completa le frasi seguenti con $i$ nomi composti scelti tra quelli elencati alla fine dell'esercizio.

a) Il ............... è un veicolo a due o a tre ruote.

b) Un'opera eccellente è anche chiamata un ................. .

c) Il valore bollato a forma di talloncino che serve per 1'affrancatura della corrispondenza spedita per posta è il ................

d) $\mathrm{La}$.............. è un armadio o una cassetta di acciaio particolarmente resistente, per custodire denaro o preziosi. 
e) Il ................ può essere un facchino o una struttura applicata esternamente a un veicolo per sostenere i bagagli durante il viaggio.

f) Una persona che è priva di una casa dove dormire è un/a

g) Un/a ............... turba l'allegria di una festa o manda all'aria piani stabiliti.

h) Un militare di grado intermedio tra la truppa e gli ufficiali è chiamato un ...................

i) Un ponte che passa sopra una o più strade o sopra una linea ferroviaria è un

l) Il postino può essere chiamato anche

senzatetto, ciclomotore, cassaforte, cavalcavia, capolavoro, portabagagli, portalettere, guastafeste, francobollo, sottufficiale.

4. Cambia le frasi dell'esercizio tre dal singolare al plurale.

5. Sostituisci le parole sottolineate con altre sottoelencate.

a. [...] volare distesa verso le valli $(\mathrm{r} .3-4)$

b. Incantevolmente cristiana fu l'ospitalità dei contadini là presso (r.9)

c. Ricorderò per molto tempo ancora la ragazzina (r.13-14) 
d. Antri profondi, fessure rocciose dove una scaletta di pietra si sprofonda in un'ombra senza memoria (r.22-23)

e. Ora hanno rivestito la sua cappella (r.33-34)

f. La semplicità bizzarra del disegno bianco risalta quando l'oro del tramonto tenta versarsi dall 'invetriata (r.36-37)

g. E corre la memoria ancora alle signore gentili dalle bianche braccia ai balconi laggiù (r.50-51)

adesso, lontano e in basso, nel momento in cui, in direzione delle, a lungo, di nuovo, vicino, nel luogo in cui.

6. Cambia dalla forma implicita a quella esplicita.

a. Io vidi dalle solitudini mistiche staccarsi una tortora e volare distesa verso le valli (r.3-4)

b. Il paesaggio cristiano segnato di croci inclinate dal vento ne fu vivificato misteriosamente (r.4-5)

c. Il corridoio, alitato dal gelo degli antri, si veste tutto della leggenda Francescana (r.29-30)

d. Ora hanno rivestito la sua cappella scavata nella viva roccia (r.33-34)

e. Bianchi sul tono ricco del noce sembrano rilevarsi i profili ieratici (r.39-40)

f. Lontano si vedono lentamente sommergersi le vedette mistiche e guerriere dei castelli del Casentino (r.47-48) 
7. Riempi gli spazi vuoti, scegliendo una delle tre proposte sottoelencate.

Nelle primissime ore del 15 settembre 1224, mentre Francesco pregava in un luogo (1) del Monte vide

(2) dal cielo un Serafino con sei ali infuocate e risplendenti, due alle spalle, due ai fianchi, e due alle gambe. Tra le ali era l' (3) di un uomo crocefisso e Francesco (4) meravigliato poiché sapeva bene che il dolore della passione non si concilia in (5) modo con la beatitudine di un Serafino. Alla fine (6) però che Dio (7) annunciava la totale trasformazione che stava per (8) nel suo corpo e che l'avrebbe fatto simile a Cristo crocefisso. [...] Quando la (9) scomparve cominciarono ad (10) nelle mani e nei piedi di Francesco i segni dei chiodi. Anche il fianco destro era (11) da una ferita rossa dalla quale spesso usciva (12) da macchiargli la tunica $[\ldots]$.

Attraverso le stimmate Francesco si identificò (13) Cristo e non più (14) spiritualmente, ma (15) fisicamente. La Verna fu (16) lui il Tabor della (17) trasfigurazione, ma anche il Golgota della sua sofferenza.

(da B. DOZZINI, Giotto. La leggenda francescana nella Basilica di Assisi, Assisi, Editrice Minerva, 1997) 
1 a) appartato

b) solo

c) insolito

2 a) volare

b) viaggiare

c) scendere

3 a) aspetto

b) emblema

c) immagine

4 a) guardò

b) rimase

c) sentì

5 a) alcun

b) tutto

c) ogni

6 a) comprese

b) imparò

c) notò

7 a) 10

b) $\mathrm{le}$

c) $\mathrm{gli}$

8 a) dare

b) avvenire

c) fare

9 a) visione

b) figura

c) vista

10a) arrivare

b) apparire

c) accadere

11a) bendato

b) coperto

c) visto

12a) liquido

b) acqua

c) sangue

13a) con

b) da

c) per

14a) anche

b) soltanto

c) come

15a) proprio

b) sempre

c) anche

16a) per

b) di

c) con

17a) propria

b) vera

c) sua.

8. Spunti per la produzione orale e scritta.

a) Una pagina dal mio diario.

b) Una visita ad una città toscana o umbra.

c) Se tu dovessi fare un viaggio per passare le ferie, che tipo di vacanza ti piacerebbe fare?

d) Descrivi una casa di campagna. 
e) Col passar degli anni, molti contadini hanno preferito lasciare $\mathrm{i}$ campi e sistemarsi nelle grandi città. Fino a che punto credi che sia stata una saggia decisione?

f) Leggi e commenta il Cantico di frate Sole di San Francesco che trovi qui di seguito.

\section{OLTRE IL BRANO}

\section{Cantico di frate Sole}

Secondo le antiche fonti francescane il Cantico sarebbe stato composto nella chiesetta di San Damiano, presso Assisi, nel 1224, dopo una notte di tribolazioni al termine della quale una visione divina avrebbe promesso a Francesco la beatitudine eterna. Il componimento è in versetti di intonazione biblica, assonanzati ma non riconducibili ad un metro preciso, e con tutta probabilità seguenti il cursus; scritto in volgare umbro, ma con una veste non decisamente dialettale tranne alcune precise o interessanti particolarità. $[\ldots]$

Anzitutto leggiamo per intero e commentiamo il testo, secondo la più recente e accreditata ricostruzione critica, ad opera del Contini : 
Altissimu,${ }^{1}$ onnipotente, bon Signore, tue so ${ }^{2}$ le laude, la gloria e l'honore et onne benedictione.

Ad te solo, Altissimo, se konfano, ${ }^{3}$

et nullu homo ène dignu te mentovare. ${ }^{4}$

Laudato sie, mi' Signore, cum tucte le tue creature, ${ }^{5}$ spetialmente messor lo frate sole, lo qual'è iorno, ${ }^{6}$ et allumini noi per lui. ${ }^{7}$

Et ellu è bellu e radiante ${ }^{8}$ cum grande splendore : de te, Altissimo, porta significatione. ${ }^{9}$

Laudato si', mi' Signore, per $^{10}$ sora luna e le stelle : in celu l'ài formate clarite ${ }^{11}$ et pretiose et belle.

Laudato si', mi' Signore, per frate vento et per aere et nubilo ${ }^{12}$ et sereno et onne tempo,

${ }^{1}$ Altissimu : con la $-u$ finale, tipicamente umbra, non riappare successivamente ; vedi poi mullu, dignu, e quindi messor per "messere", ecc.

2 tue so': sono tue, spettano a Te.

${ }^{3}$ se konfano: si confanno, si addicono.

${ }^{4}$ et nullu ... mentovare : e nessun uomo è degno di menzionarti.

${ }^{5}$ cum tucte le tue creature : così come tutte le tue creature ( $\mathrm{o}$, secondo altri, cum vale "da").

${ }^{6}$ è iorno: è luce diurna.

${ }^{7}$ et allumini ... lui : e illumini noi per mezzo di lui.

${ }^{8}$ radiante : raggiante, splendente.

${ }^{9}$ de te ... signifcatione : reca, contiene il tuo segno, o Altissimo.

${ }^{10} \mathrm{per}$ : varie sono le interpretazioni che sono state date di questo e dei successivi per, ma la più accettabile è la più semplice : per casuale, come il propter latino : “a causa di", "a motivo di".

11 clarite: splendenti.

${ }^{12}$ nubilo : le nuvole. 
per lo quale a le tue creature dài sustentamento.

Laudato si', mi' Signore, per sor'aqua,

la quale è multo utile et humile et pretiosa et casta.

Laudato si', mi' Signore, per frate focu,

per lo quale ennallumini ${ }^{13}$ la nocte :

ed ello è bello et iocundo et robustoso et forte.

Laudato si', mi' Signore, per sora nostra matre terra, la quale ne sustent ${ }^{14}$ et governa, et produce diversi fructi con coloriti flori et herba.

Laudato si', mi' Signore, per quelli ke perdonano per lo tuo amore et sostengo ${ }^{15}$ infirmitate et tribulatione.

Beati quelli ke 'l sosterrano in pace, $\mathrm{ka}^{16}$ da te, Altissimo, sirano incoronati.

Laudato si', mi' Signore, per sora nostra morte corporale, ${ }^{17}$ da la quale nullu homo vivente pò skappare :

guai a cquelli ke morrano ne le peccata mortali ; beati quelli ke trovarà ne le tue sanctissime voluntati, ka la morte secunda ${ }^{18}$ no '1 farrà male.

13 ennallumini : illumini.

14 sustenta: sostiene, alimenta (e altrettanto vale governa).

15 sostengo: sostengono.

${ }_{17}^{16} \mathrm{ka}$ : poiché.

17 morte corporale: morte del solo corpo.

${ }^{18}$ la morte secunda: la morte dell'anima, la dannazione. 
Laudate ${ }^{19}$ et benedicete mi' Signore et rengratiate e serviateli ${ }^{20}$ cum grande humilitate ${ }^{21}$.

L'intento che si proponeva Francesco nel dettare il Cantico era anche di natura pratica : offrire ai suoi fraticelli un testo da cantare a lode del Signore e da insegnare alla gente devota. Ciò spiega la semplicità d'impianto dell'inno, il ricorso a cose grandi e piccole della natura e a concetti facilmente comprensibili dai devoti.

(da G. PETROCCHI, La letteratura religiosa in Storia della letteratura italiana, vol. I, Milano, Garzanti, 1987, pp. 665-667)

Opere di DINo CAMPANA (Marradi, Firenze, 1885 - Castel Pulci, Firenze, 1932)

Canti orfici, 1914 ; Inediti, postumi, 1942 ; Taccuino, postumo, 1949 ; Canti orfici e altri scritti, postumi, 1952 ; Lettere, postume, 1958 ; Taccuinetto faentino, postumo, 1960 ; Il più lungo giorno, postumo, 1973.

${ }^{19}$ Laudate : ora il Santo si rivolge direttamente ai fedeli.

${ }^{20}$ serviateli : servitelo.

${ }^{21}$ Cfr. ed. di G. Contini, Poeti del Duecento, Milano-Napoli 1960, t. I, pp.33-34. 


\section{BIBLIOGRAFIA}

Alessandri M.R., Manuale del fantastico, Firenze, La Nuova Italia, 1992.

Annaratone C. / Rossi M.T., Lingua e società, Bologna, Zanichelli, 1977.

Baricco A., Totem, RAIDUE, 28 dicembre 1998.

Bassani G., Il giardino dei Finzi-Contini, Torino, Einaudi, 1972.

Bassani G., L'airone, Milano, Mondadori, 1971.

Binni W. / Ghidetti E. / Andreoli F., Immagini del reale, Palermo, Palumbo, 1984.

Campana D., Canti orfici, Milano, Marcos y Marcos, 1989.

Caproni G., Tutte le poesie, Milano, Garzanti, 1999.

Dardano M. / Trifone P., Grammatica italiana, Bologna, Zanichelli, 1988.

Dozzini B., Giotto. La leggenda francescana nella Basilica di Assisi, Assisi, Editrice Minerva, 1997.

Ensemble Micrologus, Calendimaggio di Assisi : Musica, Rito, Storia e Arte, Micrologus Edizioni Discografiche, 1997.

Frescaroli A., Saper scrivere bene oggi, Milano, Giovanni De Vecchi Editore, 1996.

Gabrielli A. et alii (a cura di), Come parlare e scrivere meglio, Milano, Selezione dal

Reader's Digest, 1975.

Gadda C.E., La cognizione del dolore, Milano, Garzanti, 1999.

Ginzburg N., Opere, vol. I, Milano, Mondadori, 1986.

Leone de Castris A., Storia di Pirandello, Bari, Laterza, 1978.

Marchi C., Impariamo l'italiano, Milano, Rizzoli, 1984.

Marotta G., L'oro di Napoli, Milano, Rizzoli, 1996.

Petrocchi G., La letteratura religiosa in Storia della letteratura italiana. Le origini e il Duecento, Milano, Garzanti, 1987.

Pirandello L., Il fu Mattia Pascal, a c. di Claudio Toscani, Milano, Mondadori, 1990.

Piovene G., Viaggio in Italia, Milano, Baldini \& Castoldi, 1993.

Saba U., Antologia del "Canzoniere", Torino, Einaudi, 1972.

Silone I., Fontamara, Milano, Mondadori, 1973.

Tomasi di Lampedusa G., Il Gattopardo, Milano, Feltrinelli, 1990. 


\section{INDICE}

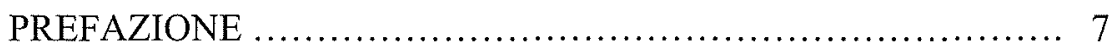

CAPITOLO 1 ................................................. 13

Casa al mare (Natalia Ginzburg)

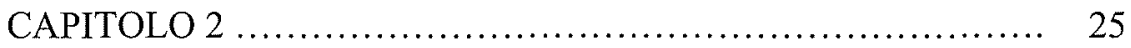

L'Umbria (Guido Piovene)

CAPITOLO 3 ............................................. 43

Tre vie (Umberto Saba)

CAPITOLO 4 ............................................ 57

La fattoria di Rampinzeri (Giuseppe Tomasi di Lampedusa)

CAPITOLO 5 ............................................. 73

I "Quartieri" (Giuseppe Marotta)

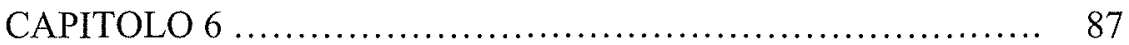

Voglio la terra (Ignazio Silone)

CAPITOLO 7 ................................................ 99

Prudenza della guida (Giorgio Caproni)

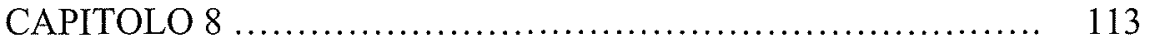

Un po' di nebbia (Luigi Pirandello)

CAPITOLO 9

L'airone (Giorgio Bassani)

CAPITOLO 10

E alle stecche delle persiane già l'alba (Carlo Emilio Gadda)

CAPITOLO 11

La Verna (Dino Campana)

BIBLIOGRAFIA 166 
Mediante riflessioni su testi tratti da opere famose pubblicate nella prima parte del Novecento e negli anni Sessanta e Settanta, per essere precisi, si vuole spingere l'allievo, o il libero discente, ad allenarsi a individuare, a confrontare e ad apprendere espressioni consuete e non sempre consuete nel modo di parlare e di scrivere contemporaneo o attuale.

L'opera è accompagnata da un CD che contiene la registrazione degli undici brani d'autore letti da Marco Brancato che dà ai testi un ritmo interpretativo intelligente ed efficace a seconda dei contenuti e delle situazioni narrate.

Renzo Pavese 